\title{
Role of Microglia in Myelin Turnover
}

\author{
Dissertation \\ for the award of the degree \\ "Doctor of Philosophy" (Ph.D.) \\ Division of Mathematics and Natural Sciences \\ of the Georg-August-Universität Göttingen
}

submitted by

Shima Safaiyan

from

Esfahan, Iran

Goettingen 2015 


\section{Member of the Thesis Committee:}

Prof. Dr. Mikael Simons, Reviewer

Department of Cellular Neuroscience, Max Planck Institute for Experimental Medicine Department of Neurology, University of Goettingen

Prof. Dr. Alexander Fluegel, Reviewer

Department of Neuroimmunology, Institute for Multiple Sclerosis Research, University Medical Center Goettingen

Prof. Dr. Holger Reichardt,

Department of Cellular and Molecular Immunology, University Medical Center Goettingen

Date of the oral examination: 21 September, 2015 


\section{Affidavit}

I hereby declare that this Doctoral thesis entitled "Role of microglia in myelin turnover" has been written independently with no other aids or sources than quoted.

Shima Safaiyan

July, 2015

Goettingen, Germany 


\section{Contents}

\section{Contents}

List of tables ix

List of figures ix

Abbreviations

Acknowledgements

Abstract

xiii

Introduction

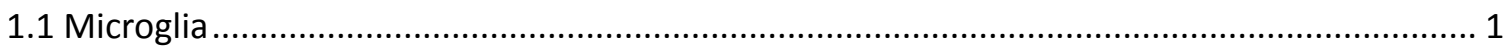

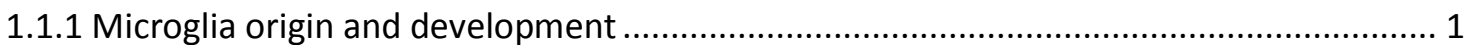

1.1.2 Microglia phenotype in central nervous system ........................................................ 1

1.1.3 Microglia function in central nervous system ............................................................ 1

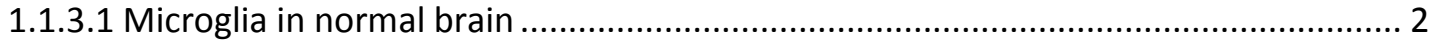

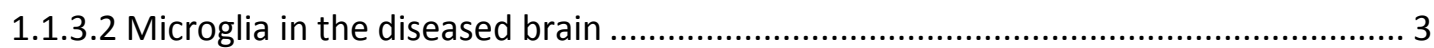

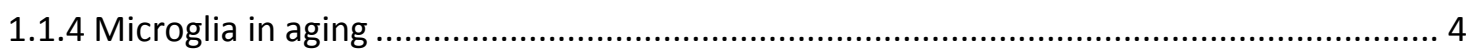

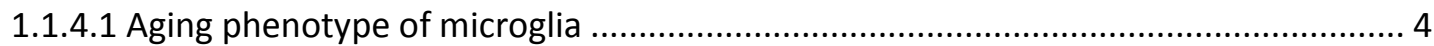

1.1.4.2 Basal activation state of microglia in aging ....................................................... 5

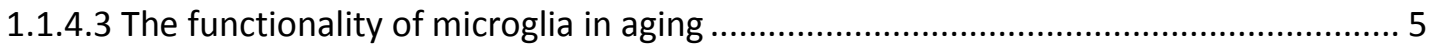

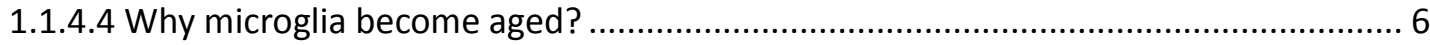

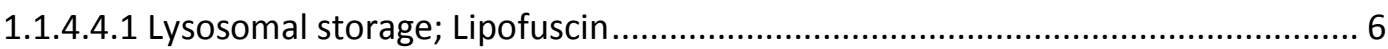

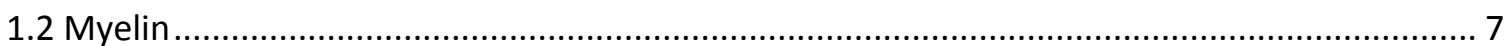

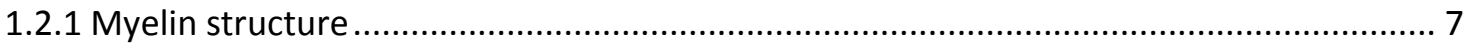

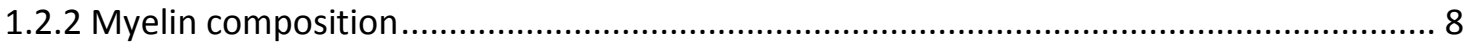

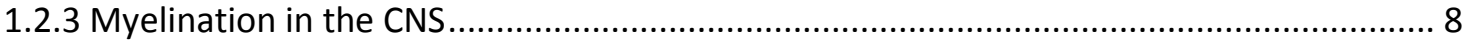

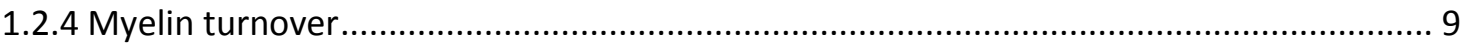

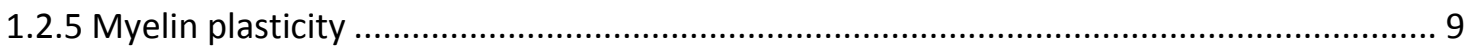




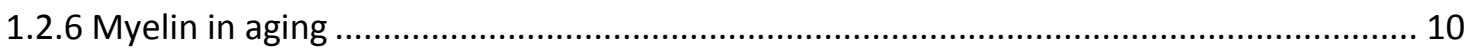

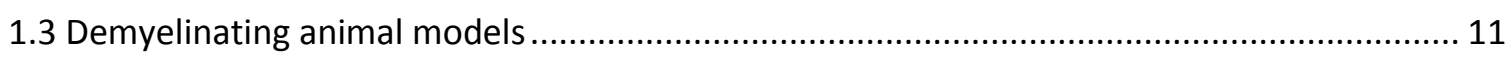

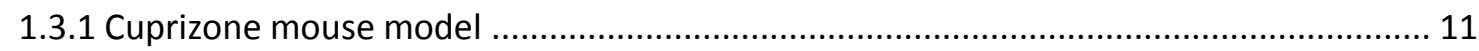

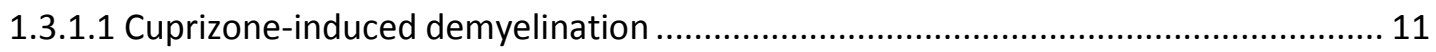

1.3.1.2 Immunopathology of cuprizone mouse model .................................................... 12

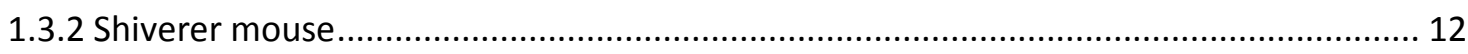

1.3.3 PLP overexpressing transgenic mice, a model for Pelizaeus-Merzbacher disease .......... 12

1.3.3.1 Microglia activation in PLP overexpressing transgenic mice................................... 13

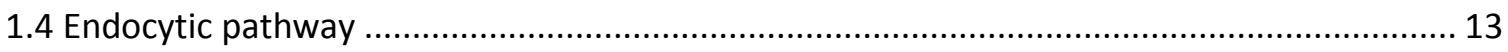

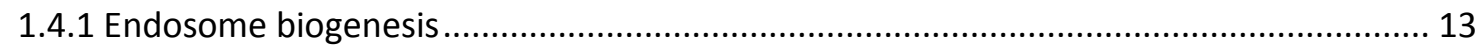

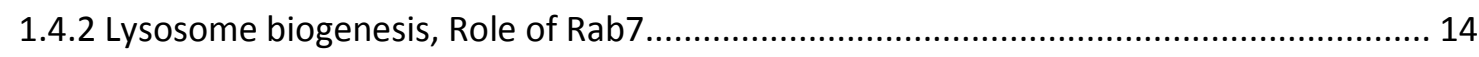

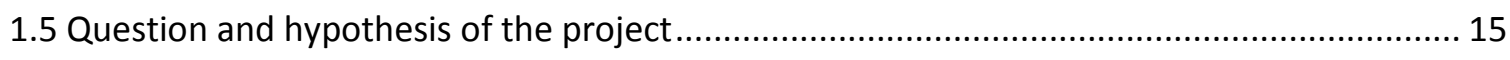

$\begin{array}{ll}\text { Materials and Methods } & 16\end{array}$

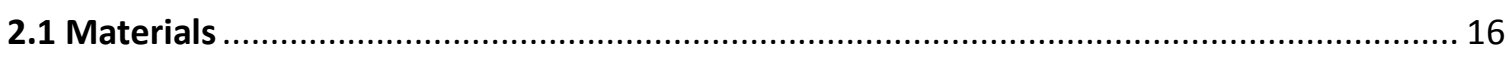

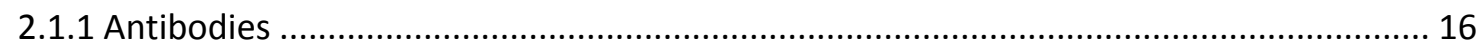

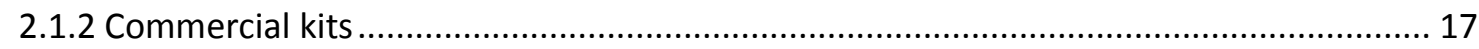

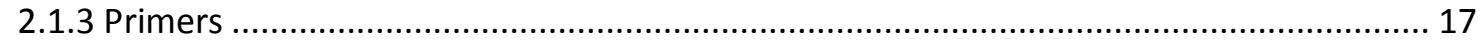

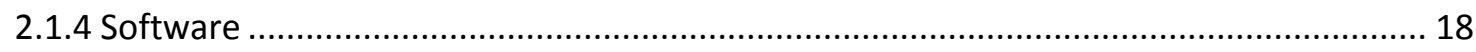

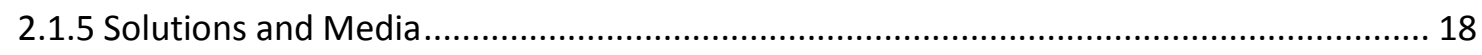

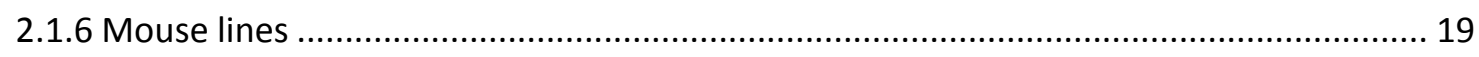

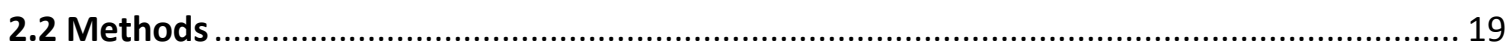

2.2.1 Generation and characterization of conditional Rab7 KO mice .................................... 19

2.2.1.1 Generation and breeding of mutant mice............................................................. 19

2.2.1.2 Tamoxifen induction of conditional deletion of Rab7 gene.................................... 21

2.2.1.3 Detection of Cre recombinase activity using reporter mice .................................. 21

2.2.1.4 Cre recombinase-mediated deletion of Rab7 gene in microglia.............................. 21

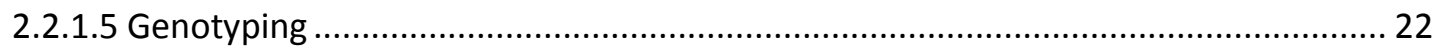




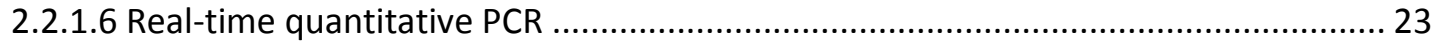

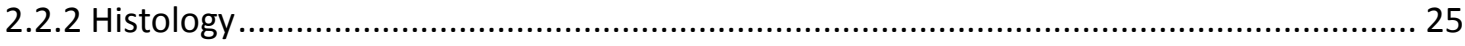

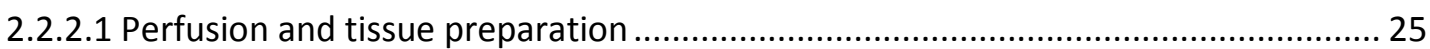

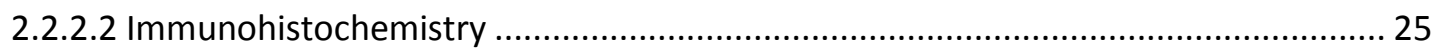

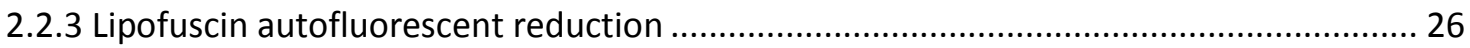

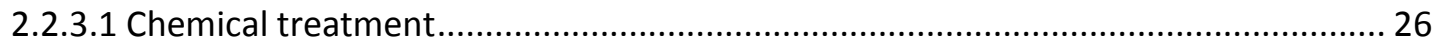

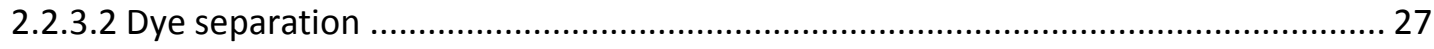

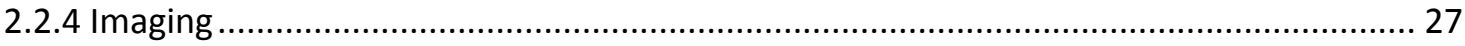

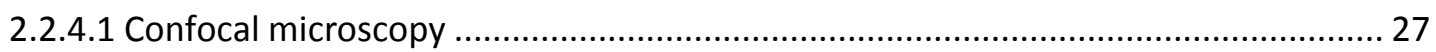

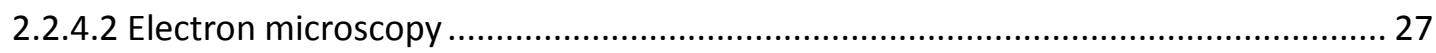

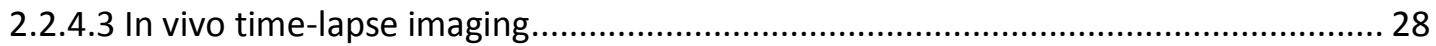

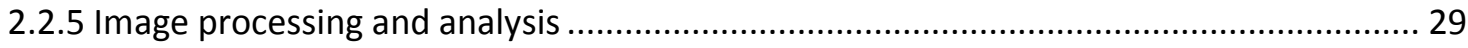

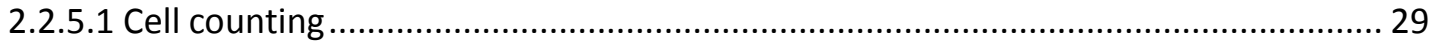

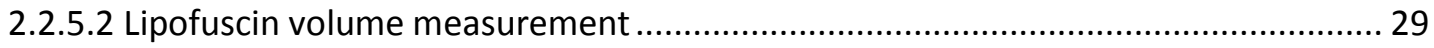

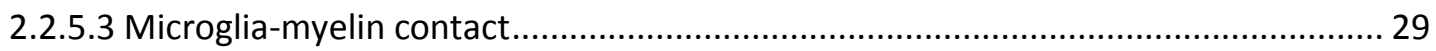

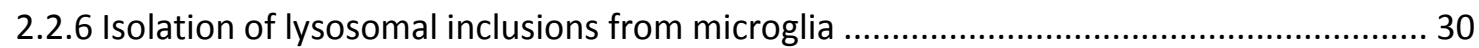

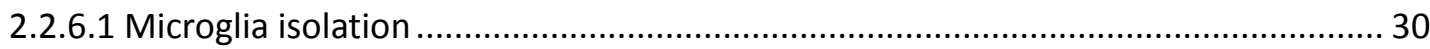

2.2.6.2 Sarkosyl-insoluble fractionation of microglia.......................................................... 31

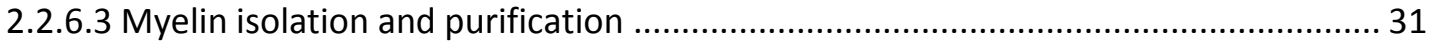

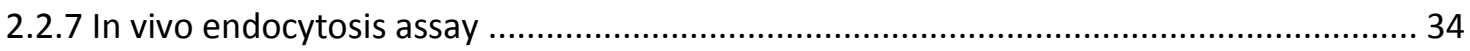

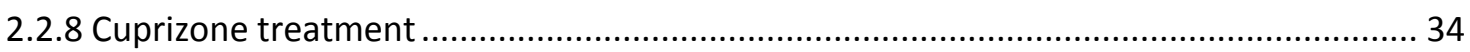

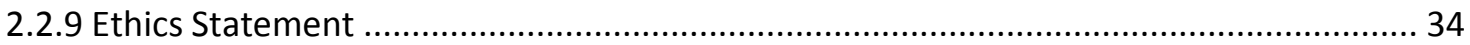

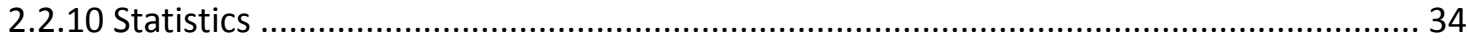

Results $\quad 35$

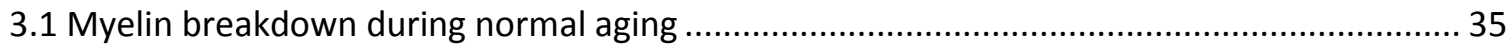

3.2 Microglia are involved in myelin turnover in normal aging ................................................ 35

3.2.1 Microglia are in contact with myelin in wild type mice ................................................. 35

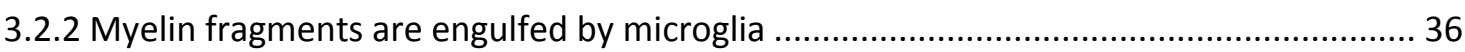


3.2.3 Density and lysosomal activity of microglia cells increase in the white matter

3.2.4 Phagocytic activity of microglia in white matter of wild type mice 39

3.3 Cellular phenotypic alterations of microglia with age 39

3.3.1 lipofuscin granules accumulate within aged microglia 40

3.3.1.1 The amount of lipofuscin in white and gray matter in aged wild type mice 41

3.3.1.2 Detection of myelin fragments within lipofuscin 41

3.3.1.3 Biochemical characterization of lipofuscin contents

3.3.2 Age-related changes in microglia shape

3.3.3 Less motility of microglia processes in aging brain related to lipofuscin accumulation .. 44

3.3.4 Age-related changes in activation state of microglia 45

3.3.5 Clearance function of microglia in aging . 46

3.4 Enhanced myelin breakdown in demyelinating models and microglia behavior 47

3.4.1 Myelin in PMD mice and cuprizone model 47

3.4.2 Microglia in PMD mice and cuprizone model 48

3.4.2.1 Morphology and activation state 48

3.4.2.2 Lipofuscin accumulation in microglia in demyelinating models 49

3.4.2.4 Clearance function of microglia in PMD mice 51

3.5 Genetically induced impairment of lysosomal degradation in microglia 52

3.5.1 Cre recombinase-mediated deletion of Rab7 gene in Rab7 knockout mice 52

3.5.2 Phenotypic characterization of conditional Rab7 knockout mice. 53

3.5.2.1 Enlarged lysosomes in microglia 53

3.5.2.2 Myelin fragments inside microglia ..................................................... 54

3.5.2.3 Early accumulation of lipofuscin in microglia............................................. 55

3.5.2.4 Myelin fragments are associated with lipofuscin within microglia....................... 55

3.5.2.5 Clearance function of microglia in conditional Rab7 KO mice ..............................56

3.5.2.6 Myelin fragments in conditional Rab7 KO mice ......................................... 57

3.5.2.7 Microglia morphology in conditional Rab7 $\mathrm{KO}$ mice ......................................... 57 
Contents

3.5.2.8 MHCll expression in microglia in conditional Rab7 KO mice

Discussion

4.1 Myelin turnover by shedding of myelin fragments into the extracellular space 59

4.2 Myelin uptake by microglia 60

4.3 Age-related increase in microglia-myelin contact is correlated with higher rate of myelin turnover in aging 61

4.4 Internalized myelin contribute to the formation of lysosomal inclusions 62

4.5 Microglial phenotype changes with age 63

4.6 Myelin breakdown leads to lipofuscin formation 64

4.7 Lysosomal storage induces cellular senescence. 66

4.8 conclusion 67

\section{Bibliography}

Curriculum vitae

83 


\section{List of tables}

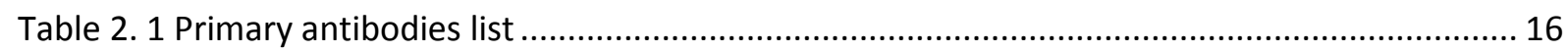

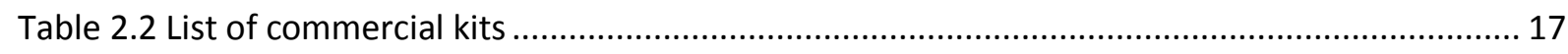

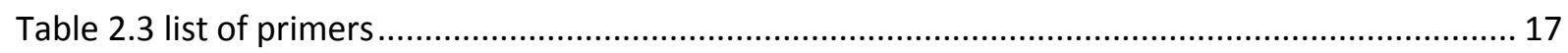

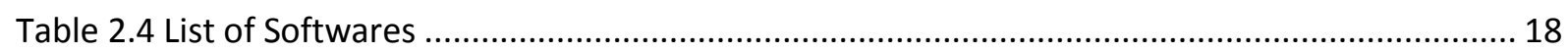

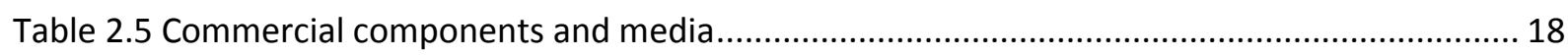

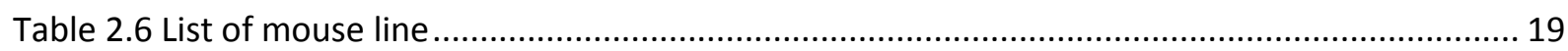

\section{List of figures}

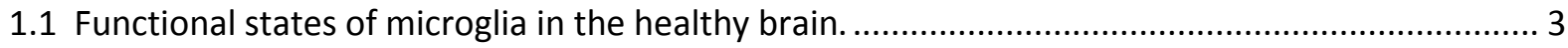

1.2 Potential types of myelin plasticity in the CNS in adulthood. ................................................ 10

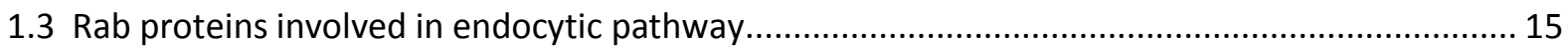

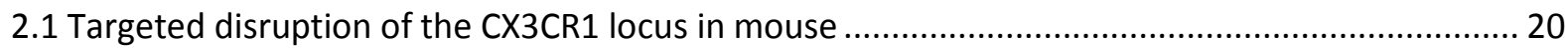

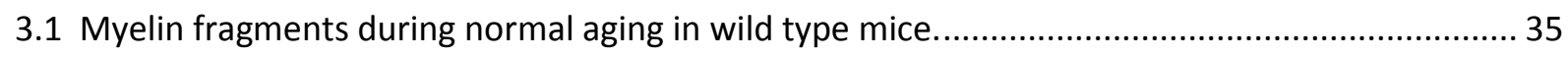

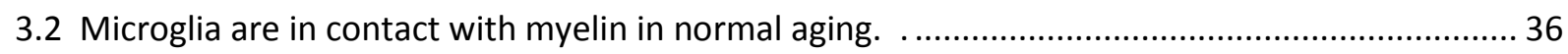

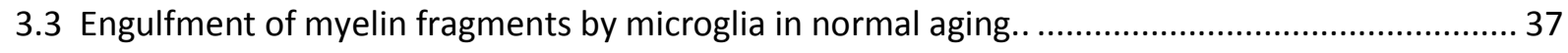

3.4 Density and lysosomal size of microglia cells increase in white matter......................................... 38

3.5 Detection of galectin 3/Mac2 positive microglial population during normal aging. .................... 39

3.6 Visualization and quantification of lipofuscin granules in wild type mouse brain......................... 40

3.7 Lipofuscin amount in white and gray matter in aged wild type mice.......................................... 41

3.8 Myelin fragments in the cells are associated with intracellular lipofuscin.................................. 42

3.9 Characterization of sarkosyl-insoluble fraction of isolated microglia........................................... 43

3.10 Aged microglia have less branched and shorter processes..................................................... 44

3.11 Dynamics of the motility of microglia processes in old and young mice .................................... 45 


\section{List of Figures}

3.12 Analysis of the activation state of microglia in normal aging................................................... 46

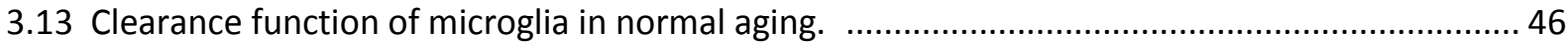

3. 14 An overview of demyelination rate in PMD and cuprizone mice......................................... 47

3.15 Morphology and activation state of microglia in PMD mice..................................................... 48

3.16 Activation state of microglia in Cuprizone mice.................................................................... 49

3.17 Lipofuscin formation and quantification in demyelinating models. .......................................... 50

3.18 Myelin fragments are found within Lipofuscin in microglia in cuprizone mice .......................... 51

3.19 Quantification of Dextran uptake by microglia in 7-month-old PMD mice. ................................ 52

3.20 Evaluation of Rab7 gene deletion in conditional Rab7 knockout mice........................................ 53

3.21 Visualization and quantification of LAMP1-positive microglia in Rab7 KO mice. ........................ 54

3.22 Myelin phagocytosis by microglia in conditional Rab7 KO mice................................................. 55

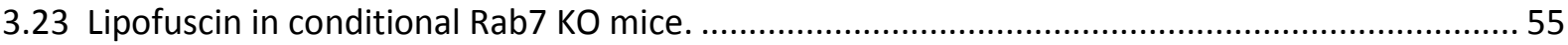

3.24 Lipofuscin contains internalized myelin fragments in microglia in Rab7 KO mice...................... 56

3.25 Clearance function of microglia in conditional Rab7 KO mice. …................................................ 57

3.26 Number of myelin fragments increases in conditional Rab7 KO mice........................................57

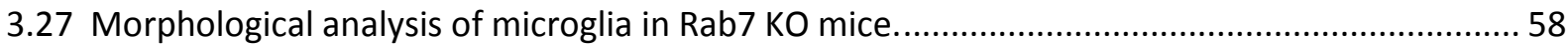

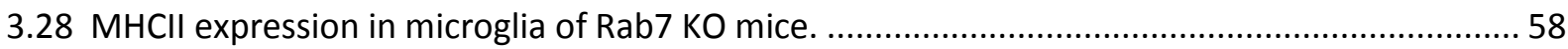

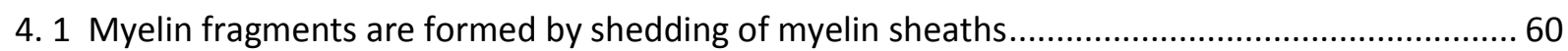




\section{Abbreviations}

\begin{tabular}{|c|c|}
\hline$A D$ & Alzheimer's disease \\
\hline APC & Antigen presenting cells \\
\hline BDNF & Brain-derived neurotrophic factor \\
\hline CCL & CC chemokines ligand \\
\hline CCR & CC chemokines receptor \\
\hline CNPase & 2', 3'-Cyclic-nucleotide 3'-phosphodiesterase \\
\hline CNS & Central nervous system \\
\hline CX3CR1 & CX3C chemokine receptor 1 \\
\hline EGFP & Enhanced Green Fluorescent Protein \\
\hline FcpRII & Fc gamma receptor II \\
\hline Iba1 & Ionized calcium-binding adapter molecule 1 \\
\hline IL-1 $\beta$ & Inteleukin 1 beta \\
\hline IL-6 & Interleukin 6 \\
\hline iNOS & Inducible Nitric Oxide Synthase \\
\hline MAG & Myelin-associated glycoprotein \\
\hline MBP & Myelin basic protein \\
\hline $\mathrm{MHCll}$ & Major histocompatibility complex II \\
\hline MOBP & Myelin-associated oligodendrocytic basic protein \\
\hline MOG & Myelin oligodendrocyte glycoprotein \\
\hline MS & Multiple Sclerosis \\
\hline MS & Mass spectrometry \\
\hline mtDNA & Mitochondria DNA \\
\hline Nef3 & Neurofilament 3 \\
\hline NF $\kappa B$ & Nuclear factor kappa-light-chain-enhancer of activated B cells \\
\hline OPC & Oligodendrocyte progenitor cell \\
\hline PLP & Proteolipid protein \\
\hline PMD & Pelizaeus-Merzbacher disease \\
\hline PNS & Peripheral nervous system \\
\hline POS & Photoreceptor outer segments \\
\hline ROS & Reactive oxygen species \\
\hline RPE & Retinal pigment epithelial \\
\hline Tag1 & Transient axonal glycoprotein-1 \\
\hline TBI & Traumatic brain injury \\
\hline TLR4 & Toll-like receptor 4 \\
\hline TNF- $\alpha$ & Tumor necrosis factor alpha \\
\hline
\end{tabular}




\section{Acknowledgements}

I would like to thank my supervisor Prof. Mikael Simons for giving me the opportunity to join his group and supporting me during my PhD.

I also thank, Prof. Alexander Fluegel and Prof. Holger Reichardt, the members of my thesis committee for their helpful advice during my PhD.

I appreciate the funding and educational support by the Max Planck Society, SFB transregio 43, the Gottingen Graduate School for Neurosciences, Biophysics, and Molecular Biosciences (GGNB) and the Georg-August University.

I am grateful to Prof. Uwe-Karsten Hanisch's group (Hana and Nasrin) for teaching me their techniques and being so friendly to share their knowledge with me.

I also appreciate all the help from our collaborator: Alexander Fluegel and his nice PhD student, Tanja Litke

I am really thankful to Jennifer Schindler, Steffi Thiel, Nadine Jagaschewskie, and Olivia Schulze, in the animal house for taking care of my mice line so carefully that I could do all my in vivo experiments without any problem during my PhD

I also should thank to Dr. Ursula Fünfschilling, and Mr. Rainer Libal in animal house for teaching me how to work with mice and helping me in different situations.

I would like to mention the colleagues and friends in AG Simons, for the warm and friendly working atmosphere and also for their help and fruitful advice during my PhD: Giselheid Schulz, Lena Steshenko, Dirk Fitzner, Nicolas Snaidero, Aniket Ghosh, Mostafa Bakhti, Sebastian Schmitt, Shweta Aggarwal, Tina Kling, Schanila Nawaz, Nils Halbsgut, Ulrich weikert, Marie-Theres Weil, Maryam Khojasteh, Caroline Velte, Ludovico Cantuti, Sebastian Timmler, Jan-Georg I specially appreciate all the support and friendship from Caro, Tina, Maryam, Mostafa, and Natalia. My Mother for her love, great support, and patience to encourage me for improving my life and education.

Alejandro, my dear, for sharing very nice moments with me and for his help and patience during last year.

My best friends in Iran, for not forgetting me and always staying in touch with me to support me emotionally in every situation, and I specially would like to thank my best best friends ever, Zizi anh Hiva in Canada, for taking care of me emotionally, giving me the strong confident to be able to deal with all my problems during all these years. 


\section{Abstract}

During development oligodendrocytes in the central nervous system (CNS) produce large amount of membrane to generate myelin which wraps around the axons. The synthesis, maintenance and turnover of such enormous amounts of myelin membranes are crucial for the maintenance of functional nerves. How molecules within the numerous layers of tightly compacted membrane get access to the degradation system for myelin turnover is not well known. In this work, we observed that microglia are in contact with myelin via their processes, and myelin fragments, generated due to myelin breakdown, are engulfed by these cells at 18 and 24 months of age. We could show that microglia are actively engaged in clearing away degenerated myelin in normal aging.

To investigate the connection between myelin breakdown and phenotypic changes in microglia, demyelinating mouse models were analysed. The results showed that microglia in these mice specifically develop large amount of lysosomal inclusions that include internalized myelin components.

In general, such inclusions are typically observed in aged post-mitotic cells and are called aging pigment or lipofuscin.

To test whether impairing the lysosomal system is sufficient to induce an aging phenotype, we generated mice in which the lysosomal degradation pathway was specifically blocked in microglia. This was achieved by deleting the gene encoding Rab7 which is essential for maturation of lysosomes. In these mice, aged microglia with large inclusions was induced already when the mice were 10 weeks old. Already at 5 months of age the clearance function impaired and the number of extracellular myelin fragments increased. Using Rab7 KO mice we confirmed the role of microglia cells in clearing myelin in normal aging, and also induced a phenotype related to aging.

These findings indicate that myelin breakdown can lead to lysosomal inclusion and impairs the clearance function of microglia.

Since the clearance function is important for the cell function to fight against aging, our results may have important implication for multiple sclerosis and neurodegenerative disease associated with protein aggregation such as Alzheimer's disease. 



\section{Chapter 1}

\section{Introduction}

\subsection{Microglia}

\subsubsection{Microglia origin and development}

Microglia are resident phagocytic cells in the central nervous system (CNS) which form $5-20 \%$ of the total glial cell population (non-neuronal cells) (Lawson, Perry et al. 1990; Perry 1998). Microglia originate from myeloid progenitors in the yolk sac before embryonic day 8; they migrate and proliferate in the parenchyma during development. (Alliot, Godin et al. 1999; Ginhoux, Greter et al. 2010; Ginhoux, Lim et al. 2013; Prinz and Priller 2014). Microglia are long-lived cells and their population is maintained in the CNS by self-renewing in physiological condition during adulthood (Lawson, Perry et al. 1992; Ajami, Bennett et al. 2007).

\subsubsection{Microglia phenotype in central nervous system}

In normal conditions, microglia are in their ramified shape with long and highly branched processes. In this stage, the cells are considered to be in the "resting" state when they express macrophagespecific molecules like Iba1 (ionized calcium-binding adapter molecule 1), F4/80 and CX3CR1 (CX3C chemokine receptor 1 or Fractalkine receptor) (Kettenmann, Hanisch et al. 2011; Prinz, Priller et al. 2011). During injury due to the loss of brain homeostasis, microglia become activated and change their morphology to an amoeboid form with retracted and less complex processes. In addition, their gene expression profile changes and pro-inflammatory cytokines like TNF- $\alpha$ (Tumor necrosis factor alpha), IL-1ß (Interleukin 1 beta), and IL-6 (Interleukin 6) as well as activation markers such as MHCII (major histocompatibility complex II), FcyRII (Fc gamma receptor II), and iNOS (inducible Nitric Oxide Synthase) are expressed (Kettenmann, Hanisch et al. 2011).

\subsubsection{Microglia function in central nervous system}

Microglia cells have a variety of functions including surveillance, pruning, phagocytic and inflammatory activities which are important for the maintenance of CNS homeostasis during 


\section{1 | Introduction}

development, adulthood, and aging in healthy and injured brain (Perry, Nicoll et al. 2010; Ginhoux, Lim et al. 2013; Perry and Teeling 2013; Gomez-Nicola and Perry 2015).

\subsubsection{Microglia in normal brain}

The classical view about microglia was that when they are in their resting state with the ramified morphology, they shut down most of their functions. However, using in vivo two photon imaging in the neocortex it has been shown that the cells are highly motile in this stage, actively scanning the environment in the healthy CNS (Nimmerjahn, Kirchhoff et al. 2005). Based on this finding, microglia are thought to actively survey the CNS using their processes and phagocytic activity to remove damaged tissues, cells and toxic substances even in the so-called "resting' state (Tremblay, Stevens et al. 2011; Gomez-Nicola and Perry 2015). However, beside surveillance function, microglia have additional functions such as contributing to brain plasticity and maintaining the integrity of the CNS (Lourbopoulos, Erturk et al. 2015). For example, by secreting cytokines and neurotrophic factors such as BDNF (Brain-derived neurotrophic factor), microglia cells increase plasticity of synapses which are related to learning and memory (Parkhurst, Yang et al. 2013). Furthermore, live imaging of fluorescent-labeled neurons and microglia has shown that microglia are in contact with synapses in the steady-state condition, and their contact increases when neurons are more active suggesting that microglia cells are important for neuronal circuitry and connectivity (Wake, Moorhouse et al. 2009). Using electron microscopy and two-photon in vivo imaging in the primary visual cortex, it was shown that microglia play a role in the remodeling of synaptic structures. Microglia processes are in contact with axon terminal and dendritic spine, where the synapses are located, to modify the structure of synapses by remodeling the extracellular environment. In addition, synaptic elements have been detected inside microglia in normal condition suggesting that these cells are part of the degradation system for elimination of synaptic components (Wake, Moorhouse et al. 2009; Tremblay, Lowery et al. 2010; Tremblay and Majewska 2011; Salter and Beggs 2014). Moreover, in vitro studies have shown that exosomes or extracellular microvesicles derived from oligodendrocyte membranes are exclusively internalized by microglia. In addition, when the purified exosomes were injected in the mouse brain they were engulfed by microglia suggesting that microglia may be responsible for the degradation of oligodedrogial membrane (Fitzner, Schnaars et al. 2011). Different functions of microglia in healthy brain are shown in figure 1.1. 


\section{Systemic sensing microglia}

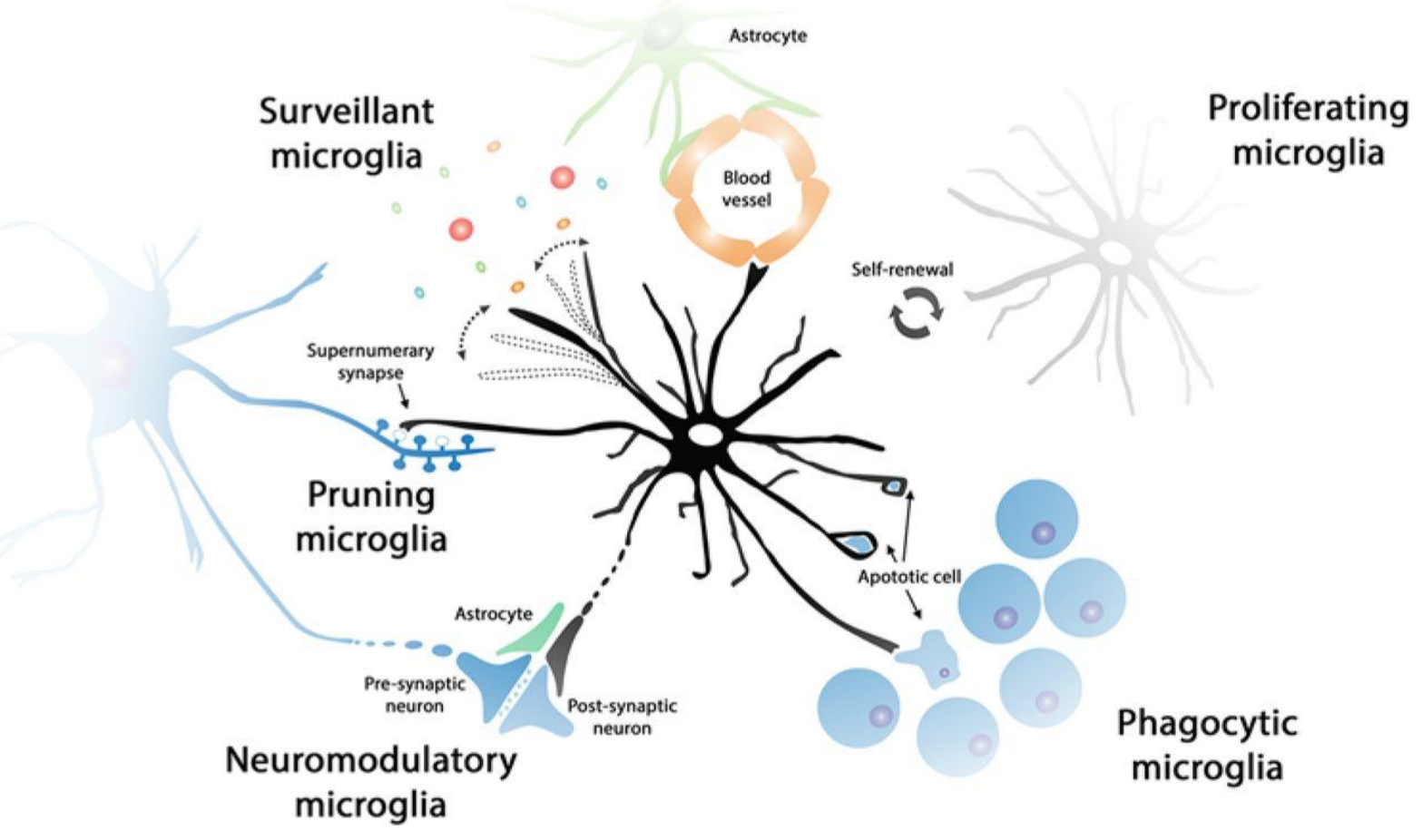

Figure 1.1 Functional states of microglia in the healthy brain. Phagocytic cells remove apoptotic neurons and tissue debris, this function is important for high turnover of cell population and consequently for maintaining the CNS integrity. Microglia are also important for removing extra synapses by pruning axon terminal. Furthermore, microglia modulate neuronal activity at the level of synapses. Diego Gomez-Nicola, and V. Hugh Perry, Neuroscientist 2014; 1073858414530512. Copyright by SAGE Publications.

\subsubsection{Microglia in the diseased brain}

Microglia have been considered as the brain immune cells contributing in the first line of defense in acute lesions and against microbes by clearing off damaged tissues and cells. These cells are involved in brain injuries and disorders via their phagocytic activity and neuroinflammatory responses (Banati 2003; Nimmerjahn, Kirchhoff et al. 2005; Kettenmann, Hanisch et al. 2011). However, it is notable that due to the limits for distinguishing resident microglia from infiltrating cells it has not been easy to identify the exclusive role of microglia in disease condition. Now, an increasing number of studies indicate that the function of short-lived peripheral macrophages and long-lived resident microglia are distinguishable (Butovsky, Jedrychowski et al. 2014; Evans, Barkauskas et al. 2014; Prinz and Priller 2014). For example, in demyelinating model such as EAE (Experimental autoimmune encephalomyelitis), resident microglia are thought to be protective by clearing off the debris whereas infiltrating macrophages appear to cause demyelination due to proinflammatory responses (Yamasaki, Lu et al. 2014). The expression profile of activated microglia, including proinflammatory 


\section{1 | Introduction}

and anti-inflammatory cytokines and other mediators varies depending on the insult in unhealthy brain. The functionality of activated cells can be beneficial or detrimental (Gomes-Leal 2012; Heneka, Kummer et al. 2014; Perry and Holmes 2014). Neuroinflammatory responses are the main reaction of activated microglia in the pathology of most neurodegenerative diseases. For instance, microgliamediated neuroinflammation causes formation of neurofibrillary tangles, pathological hallmark of the Alzheimer's disease (AD). Moreover, microglial immune responses, such as IL-1 $\beta$ expression, are suggested to be sufficient for tau pathology. (Kosik, Joachim et al. 1986; Iqbal, Grundke-lqbal et al. 1989; Iqbal, Alonso Adel et al. 2005; Bhaskar, Konerth et al. 2010; Heneka, Kummer et al. 2014). On the other hand, microglia cells remove extracellular $\beta$-amyloid accumulated in AD brain (Lee and Landreth 2010). It has been studied that TLR4- (Toll-like receptor 4) and TNF-mediated activation of microglia are involved in the loss of dopaminergic neurons in the mid brain of Parkinson's disease models. (McCoy, Ruhn et al. 2008; Noelker, Morel et al. 2013).

\subsubsection{Microglia in aging}

Considering the fact that microglia reside in the CNS during lifetime of an animal, they are susceptible to aging (Ajami, Bennett et al. 2007; Mildner, Schmidt et al. 2007). Therefore, an increasing number of studies focus on the anatomical, morphological, physiological, and molecular changes that microglia undergo in normal aging (Conde and Streit 2006; Wong 2013).

\subsubsection{Aging phenotype of microglia}

It has been suggested that the functionality of microglia decline during aging (Streit and Xue 2010). To keep the overall function, microglia are able to proliferate regionally. Therefore, in aging the density of microglia cells in different region of CNS increases (Mouton, Long et al. 2002; Damani, Zhao et al. 2011; Tremblay, Zettel et al. 2012). However, the reason for the increase in the number of cells during aging is not clear. (Wong 2013). In addition, the distribution of microglia in aged brain is regionally different (Santos, Calvente et al. 2008; Damani, Zhao et al. 2011; Tremblay, Zettel et al. 2012). In aging, the morphology of microglia also changes; their processes are shorter and show less complexity so that the surface area of the cells decreases (Sierra, Gottfried-Blackmore et al. 2007; Damani, Zhao et al. 2011; Tremblay, Zettel et al. 2012). In addition, the morphology of microglia in aged human brain is considered to be dystrophic in which the processes are segmented and deramified showing large swellings (spheroid) at the end. Notably, the dystrophic changes in microglia are not comparable to the morphological changes that occur during microglial activation in injury (Streit, Sammons et al. 2004). 


\subsubsection{Basal activation state of microglia in aging}

The phenotype of aged microglia is similar to activated cells; for example, they show hypertrophy and retracted processes (Sheng, Mrak et al. 1998; Miller and Streit 2007). In addition, activation markers such as MHCII, CD11b, and OX6 are up-regulated in aged microglia (Ogura, Ogawa et al. 1994; Frank, Barrientos et al. 2006; Ziv, Ron et al. 2006). Increased level of inflammatory cytokines such as IL1 $\beta$, TNF- $\alpha$, IL-6 is also typical of aged microglia (Sheng, Mrak et al. 1998; Sierra, GottfriedBlackmore et al. 2007; Njie, Boelen et al. 2012). The increased activated basal state of microglia in healthy aging can explain the susceptibility of aged CNS to neurodegenerative diseases (Medzhitov 2008).

\subsubsection{The functionality of microglia in aging}

Regarding age-related changes in the morphology and activation state of microglia it is reasonable to connect these alterations to possible changes in their normal tasks during aging. The dystrophic cells in aged human brain showed a decline in their neuroprotective function (Streit and Xue 2009). Furthermore, it has been found that microglia in aged brain cannot manage their role in synaptic plasticity as efficient as young cells because they are in less contact with synapse and axon terminal (Wong 2013). In fact, this deficiency can be due to declined dynamic motility of aged microglial processes (Damani, Zhao et al. 2011). Aged microglia are still able to migrate toward the injured site through parenchyma but at a lower rate compared to young cells (Damani, Zhao et al. 2011). Interestingly when they proliferate, their number does not return to the original level, and their activation state persist long term after recovery (Ajami, Bennett et al. 2007). In other words, microglia in aged brain represent an enhanced priming state so that, in unhealthy brain, they are more responsive, and their reaction is more sustained (Sparkman, Martin et al. 2005; Conde and Streit 2006; Sierra, Gottfried-Blackmore et al. 2007; Sandhir, Onyszchuk et al. 2008; Wasserman, Yang et al. 2008; Norden and Godbout 2013). The age-related priming of microglia results from dysregulated balance between signals which induce activation ("On" signals) and signals that adjust the maintenance or reversibility of responses to the basal state ("Of" signals) (van Rossum and Hanisch 2004; Biber, Neumann et al. 2007; Wong 2013). For example, while the expression of TLR1, 4, 5, 7 and CD14 is up-regulated in aging, the level of "Of" signals such as CX3CR1 and CD200 is lower (Frank, Barrientos et al. 2006; Letiembre, Hao et al. 2007; Bachstetter, Morganti et al. 2011). 


\section{1 | Introduction}

\subsubsection{Why microglia become aged?}

Aged microglia have shorter telomeres in comparison with young cells; therefore, their ability for proliferation is limited (Flanary, Sammons et al. 2007). In addition, the production of telomerase, an enzyme for extending the length of telomeres, in aged cells is low. Consequently, telomeres cannot be elongated in again after injury-induced replication (Flanary and Streit 2005). Replication senescence appears to be induced in aged microglia due to shortening of telomeres. Furthermore, an increased level of mitochondria DNA (mtDNA) has been detected in aged microglia and the respiratory chain is thought to be dysfunctional so that reactive oxygen species (ROS) are overproduced and accumulate within the cells (Corral-Debrinski, Horton et al. 1992; Lin, Simon et al. 2002). It has been suggested that the accumulation of ROS may result in elevated microglial activation during aging because ROS is important for modulation of pro-inflammatory cytokine production which is regulated by NF-KB-(Nuclear factor kappa-light-chain-enhancer of activated B cells) mediated transcription (Toledano and Leonard 1991; Nakanishi, Hayashi et al. 2011). Moreover, since microglia are long-lived cells and their physiological activities are sustained with time, the biological components accumulate within these cells. These accumulations affect the phenotype of the cells including morphology and activation state (Ma, Coon et al. 2013; Wong 2013).

\subsection{Lysosomal storage; Lipofuscin}

Lipofuscin is an indigestible polymeric compound which is formed in non-dividing cells such as neurons, retinal pigment epithelial cells (RPE) as well as microglia due to the accumulation of biological molecules and waste material with time (Essner and Novikoff 1960; Terman and Brunk 1998; Brunk and Terman 2002). Lipofuscin is yellowish-brown with autofluorescent feature; it includes various fluorophores that can be detected across a broad spectrum (Terman and Brunk 1998; Gray and Woulfe 2005). Lipofuscin formation is considered as lysosomal storage which is the main characteristic of aging phenotype since the amount of that increases with age (Strehler, Mark et al. 1959; Hohn and Grune 2013).

Lipofuscin has been detected as lysosomal inclusions in microglia in the aged brain. (Peinado, Quesada et al. 1998; Sierra, Gottfried-Blackmore et al. 2007; Tremblay, Zettel et al. 2012). However, lipofuscin formation is well-known in RPE cells. Lipofuscin in RPE cells is mainly derived from photoreceptor outer segments (POS) in the retina (Feeney-Burns and Eldred 1983). In physiological condition, regularly, RPE cells phagocytose POS (photoreceptor outer segments) in a large amounts (Bok 1993; Strauss 2005). Digestion of this huge amount of photoreceptors is a big challenge for the lysosomal system of the cells (Feeney-Burns and Eldred 1983). On the other hand, with time, the end products of oxidized low-density lipoproteins and lipid peroxidation, which are produced during 
normal cell metabolism, are stored within lysosomes. These metabolites reduce the degradation of internalized POS. Therefore, POS-derived components accumulate within the cells and facilitate the formation of lipofuscin (Finnemann, Leung et al. 2002; Kaemmerer, Schutt et al. 2007; Kaarniranta, Sinha et al. 2013). In general, it has been shown that the formation of lipofuscin is mainly due to the oxidative stress in the lysosomes. Under normal conditions, the internalized materials in the lysosomes become oxidized because of iron-catalyzed peroxidation occurring inside the lysosome. Consequently, they become resistant to enzymatic activity of lysosome and then indigestible. Then these undegradable components accumulate in the form of lipofuscin with time when the cells no longer divide (Terman and Brunk 1998; Boulton, Rozanowska et al. 2004; Terman, Kurz et al. 2010; Kaarniranta, Sinha et al. 2013). Regarding the importance of oxidative reactions for the daily life of cell, and the fact that a small amount of lipofuscin is also formed in young cells; therefore, oxidative reaction should be considered as age-independent inducer of lipofuscin formation. Age-related increase in autophagocytosis, decline in lysosomal activity, and decrease in exocytosis are noticed as age-dependent enhancer of lipofuscin (Terman and Brunk 1998).

The accumulation of lipofuscin in the cells contributes to a decline in cell function (Neufeld 1991; Brunk and Terman 2002). For example, proteosomal activity is inhibited by loading cells with lipofuscin in vitro (Sitte, Huber et al. 2000). In addition, due to high amount of iron in lipofuscin the cells become more susceptible to oxidative stress which is mainly iron-mediated (Terman, Abrahamsson et al. 1999). The accumulation of damaged mitochondria has been detected within lipofuscin-loaded cells in lysosomal storage diseases. (March, Wurzelmann et al. 1995). Furthermore, lipofuscin accumulation in RPE cells cause the most common age-associated visual disorder, known as age-related macular degeneration (Katz 2002; Wolf 2003; Sparrow and Boulton 2005); also, it can be a risk factor for the age-related diseases such as Alzheimer's and Parkinson disease (Adamec, Mohan et al. 2000; Brunk and Terman 2002; Wolf 2003; Sparrow and Boulton 2005).

\subsection{Myelin}

\subsubsection{Myelin structure}

Myelin is composed of tightly wrapped membranes around axons and is produced by oligodendrocytes in the CNS (Bunge, Bunge et al. 1962). Wrapped myelin membranes along the length of axons are regularly spaced; this space, which is myelin free, is called node of Ranvier. In fact, the compacted structure of myelin is important for the fast and saltatory conduction of electric signals from one node to the next. The myelinated area between two nodes is called internode, at each edge of internode, paranodes are located which are considered as the main region for the 


\section{1 | Introduction}

contact between axon and myelinating oligodendrocyte. The region between paranode and internode is called juxtaparanode (Pfeiffer, Warrington et al. 1993(Baumann and Pham-Dinh 2001). Myelin sheath is composed of compacted and non-compacted areas. Compacted myelin is refferd to closely packed membrane along the internode between inner and outer tongue with a thin cytoplasm. Non-compacted myelin includes paranodes, contains more cytoplasm and is important for transport of metabolites to the compacted area during myelin biogenesis (Quarles 1999; Snaidero, Mobius et al. 2014)

\subsubsection{Myelin composition}

While most plasma membranes are composed of $50 \%$ proteins and $50 \%$ lipids in dry weight, $73-81 \%$ of the total dry weight of myelin membrane is formed by lipids (Pfeiffer, Warrington et al. 1993; Chrast, Saher et al. 2011). About $27 \%$ of myelin lipids are glycosphingolipids including Galactosyceramide as well as sulfatide, and almost 16\% are plasmalogens (Aggarwal, Yurlova et al. 2011). In addition, cholesterol is the most abundant lipid in myelin (Baumann and Pham-Dinh 2001). Myelin basic protein (MBP) and proteolipid protein (PLP) account for the majority of myelin proteins (Campagnoni and Macklin 1988). These two proteins are mainly enriched in campacted myelin (Bakhti, Aggarwal et al. 2014) while Neurofascin 155 and Tag1 (transient axonal glycoprotein-1) are typical proteins of paranodes and juxtaparanodes. Myelin oligodendrocyte glycoprotein (MOG), myelin-associated glycoprotein (MAG), myelin-associated oligodendrocytic basic protein (MOBP), and 2', 3'-Cyclic-nucleotide 3'-phosphodiesterase (CNPase) compose the remaining part of myelin protein content. CNPase and MAG are found in non-compacted myelin while MOBP is included in compact myelin (Poliak and Peles 2003; Salzer 2003).

\subsubsection{Myelination in the CNS}

During development oligodendrocyte produce a huge amount of myelin membrane. One cell can form $20 \times 10^{5} \mu^{2}$ surface area of myelin (Pfeiffer, Warrington et al. 1993); in addition, oligodendrocytes can myelinate several axons at once. In the cortex and corpus callosum one cell can produce between 30 and 80 internodes ranging from 20 to $200 \mu \mathrm{m}$ in length with up to 60 different lamellae on several thin axons. On the other hand, larger axons are myelinated with longer internodes $(1500 \mu \mathrm{m})$ and thicker myelin sheath with 150 layers (Matthews and Duncan 1971; Murray and Blakemore 1980; Hildebrand, Remahl et al. 1993). Oligodendrocytes are considered as the most efficient membrane producers. In human, during the first year of age, the majority of the axons become myelinated, but in some cortical areas myelination still occurs during adulthood 
(Fields 2008). Myelination in mice starts at birth and it is completed in most areas at postnatal day 60 (Baumann and Pham-Dinh 2001).

\subsubsection{Myelin turnover}

Membrane components of myelin need to be replaced continuously to maintain myelin integrity and, consequently, nerve conductance in the CNS. Myelination is an intensive ongoing process by oligodendroctytes in development and also later during adulthood (Watkins, Emery et al. 2008; Young, Psachoulia et al. 2013; Nave and Werner 2014), removal of modified toxic components or myelin disposal, produced or accumulated during this process, is considered as a part of myelin turnover. In general, myelin components have slow turnover rate. Using stable isotope-labeling and mass spectrometry (MS) the half-replacement time of myelin lipids was measured according to incorporation rate of newly synthesized molecules in adult mice. The results showed that cholestrol with 359 days half-life is the most stable lipid in the myelin sheath. Ganglioside GM1, cerebroside, phosphatidylethanolamine, and phosphatidylcholine have a half-life of 102 days, 94 days , 25 days and 20 days, respectively (Ando, Tanaka et al. 2003). Furthermore, using pulse-chase labeling and mass spectrometry in rats, some proteins of myelin sheaths including MBP, PLP, MOG and Nef3 (Neurofilament 3) were found in the list of long-lived proteome (Toyama, Savas et al. 2013).

It is notable that turnover rate of myelin components can be age-related. For example, cerebroside and GM1 were replaced with a slightly higher rate in aged mice compared to young and adult animals (Ando, Tanaka et al. 2003).

\subsubsection{Myelin plasticity}

The concept of myelin plasticity implies that newly differentiated oligodendrocytes are added to the CNS due to the continuous division and differentiation of oligodendrocyte precursor cells (OPCS) from development to the adulthood. (Dimou, Simon et al. 2008; Rivers, Young et al. 2008; Kang, Fukaya et al. 2010; Zhu, Whittemore et al. 2011; Wang and Young 2014). The newly synthesized myelin sheaths by adult-born oligodendrocytes are suggested to be incorporated into the preexisting myelin sheath through myelin sheath replacement or myelin remodeling; or they can be used for de novo myelination of previously naked axons (Wang and Young 2014) (Figure 1.2). Furthermore, it has been shown that in comparison to oligodendrocytes in development, adult-born oligodendrocytes in the brain of P60 mice generate more and shorter internodes throughout the 


\section{1 | Introduction}

axon (Young, Psachoulia et al. 2013) and the length of internodes is even more reduced in aging in the spinal cord of 14 and 21 months old mice (Lasiene, Matsui et al. 2009) In fact, it has been suggested that generation of short internodes is an indication of de novo myelination as it happens during remyelination after myelin degeneration (Gledhill and McDonald 1977). In addition, the cell cycle time of OPCs (NG2 expressing cells) increases with age; therefore, the proliferation rate of OPCs in aging is low. (Lasiene, Matsui et al. 2009; Psachoulia, Jamen et al. 2009; Clarke, Young et al. 2012; Young, Psachoulia et al. 2013). However, some studies have shown that the number of new oligodendrocytes (CC1 positive cells) increases in aged mice and also monkeys (Sandell and Peters 2002; Lasiene, Matsui et al. 2009).

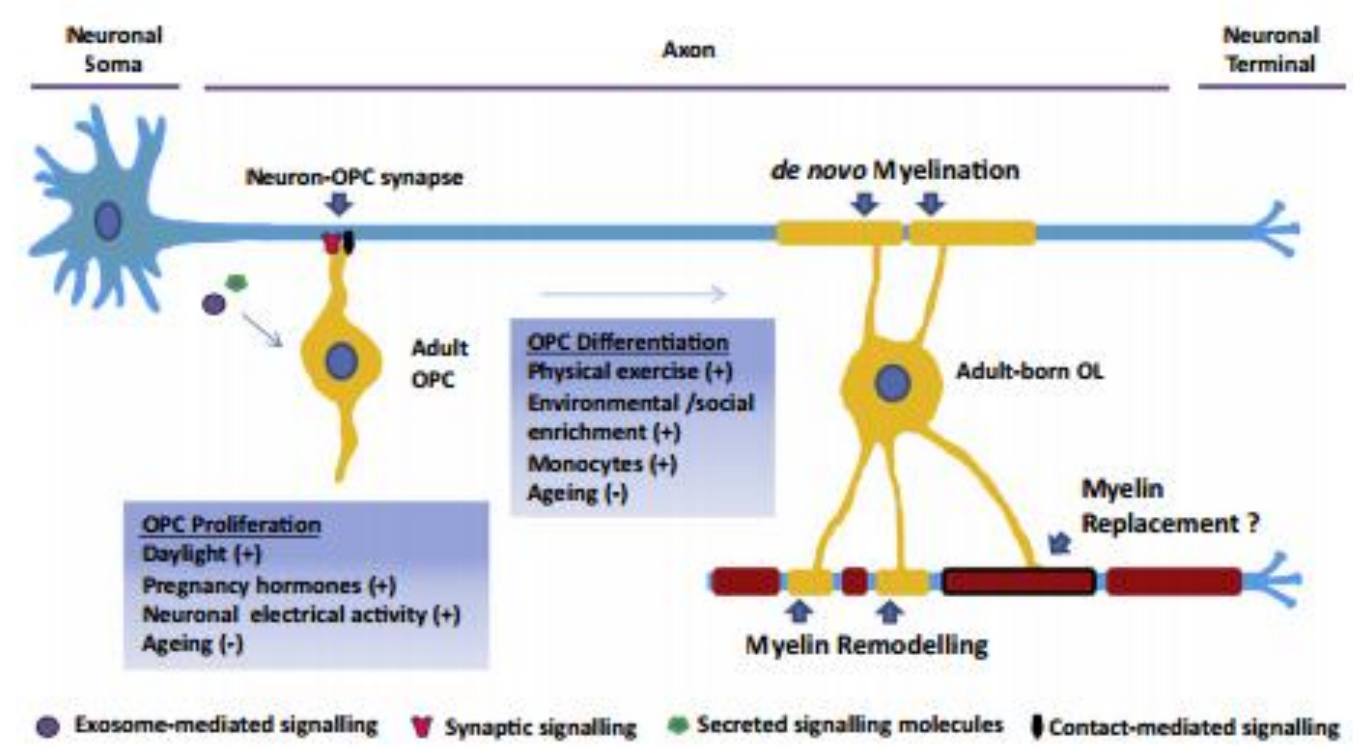

Figure 1.2 Potential types of myelin plasticity in the CNS in adulthood.

Reprint by permission from Elsevier, license number: 3653661489499

\subsubsection{Myelin in aging}

Age-related changes in myelin structure have been mostly studied by Peters et $a l$. in rhesus monkeys (Sandell and Peters 2001; Peters and Kemper 2012). Myelin alterations during aging, which are considered as degeneration of myelin membrane structure, cause changes in conduction of electric signals between neural networks (Peters and Kemper 2012). Therefore, these changes can be the main reason for cognitive decline. The most common age-dependent defect in myelin is that myelin layers break at the major dense line (formed by apposition of cytoplasmic faces at the edges of the sheet, enclosing cytoplasm within a loop (Quarles 1999)) so that an area with a dense cytoplasm containing lysosomes, vacuoles, and inclusions is formed. Another effect of aging is the formation of 
balloons in the myelin sheath. The size of these balloons varies from 1-10 micron; the large ones, basically, interfere with the loading of new formed myelin membrane in the preexisting membrane. It has been shown that in aging myelin formation is continuous which results in myelin redundancy and also the formation of thick sheath with several circuitous splits (Peters 2002; Peters 2007). Over 25 years of age in monkeys there is a decrease in the number of nerve fibers. Moreover, due to the degeneration of axons in the nerve fibers some empty myelin sheaths are detected in the primary visual cortex of a 13-year-old monkey (Sandell and Peters 2001; Peters 2002; Peters 2007).

\subsection{Demyelinating animal models}

\subsubsection{Cuprizone mouse model}

\subsubsection{Cuprizone-induced demyelination}

Cuprizone (bis-cyclohexanone-oxaldihydrazone) is a copper chelator. The administration of cuprizone in mice induce copper deficiency preferentially in oligodendrocytes and consequently demyelination. (Kesterson and Carlton 1971; Pattison and Jebbett 1971; Blakemore 1972). Copper is important for the functionality of mitochondrial enzymes including copper-zinc superoxide dismutase and ceruloplasmin (Walshe 1995). Therefore, it has been hypothesized that due to cuprizone-induced copper deficiency, mitochondrial function and consequently energy metabolism in oligodendrocytes is disturbed and cell death is induced (Komoly, Jeyasingham et al. 1987; Matsushima and Morell 2001). However, why only oligodendrocytes are susceptible is not well known. It has been thought that due to production of massive amount of myelin, these cells are metabolically active and have an high energy requirement (Matsushima and Morell 2001). Feeding the mice with $0.2 \% \mathrm{w} / \mathrm{w}$ cuprizone in the food for 3-4 weeks induce demyelination mainly in corpus callosum, 5 weeks after cuprizone diet more than $90 \%$ of axons are affected. Interestingly, 6 weeks after treatment $50 \%$ of axons become remyelinated, and by removing cuprizone, remyelination occurs continuously (Matsushima and Morell 2001; Merkler, Boretius et al. 2005). On the other hand, increasing the duration of cuprizone treatment to 10-12 weeks causes ongoing demyelination so that a few myelinated axons are detected (Hoffmann, Lindner et al. 2008). 


\section{1 | Introduction}

\subsubsection{Immunopathology of cuprizone mouse model}

The blood-brain barrier in cuprizone model is not breached; therefore, there are only little macrophages in the CNS during demyelination course. (Matsushima and Morell 2001; McMahon, Suzuki et al. 2002). 3 weeks after cuprizone treatment, when the demyelination is active, a significant number of microglia are observed mainly in corpus callosum. During 4-6 weeks of cuprizone exposure the number of activated cells increases (Hiremath, Saito et al. 1998). Beside possible infiltration of monocytes from periphery, it has been suggested that resident microglia from the other regions of the brain migrate to the lesion or they are locally proliferated (Matsushima and Morell 2001). Microglia in cuprizune-induced demyelination remove myelin debris (Smith 1999). Due to intact blood-brain barrier and minimal infiltration of peripheral immune cells, cuprizone mouse model has been considered as a suitable model to study the role of resident microglia during de/remyelination.

\subsubsection{Shiverer mouse}

Shiverer mutant mouse was first observed in the F38 generation of Swiss Vancouver stock by Biddle et al. (1973). Shiverer is an autosomal mouse mutation of MBP; there is no compact myelin in the CNS of the mouse carrying this mutation. While the normal MBP gene (32 kb) has 7 exons, 5 are deleted in shiverer mice and only the first two exons remain (Roach, Takahashi et al. 1985). Generalized tremors, seizures, and a short life span (50-100 days) are the main characteristics of this mutant (Chernoff 1981). Dysmyelination in these mice is not due to oligodendrocyte cell death, and no infiltration of macrophages has been reported (Bird, Farrell et al. 1978). The form of axoglial junctions in the shiverer CNS is not normally oriented because of fine tubular processes of oligodendrocytes around the axon. In addition, the number of these junctions increases (Rosenbluth 1981). Furthermore, the major dense line, which is normally established by cytoplasmic leaflet of the oligodendrocyte membrane in myelin is absent in the mutant mice (Privat, Jacque et al. 1979).

\subsubsection{PLP overexpressing transgenic mice, a model for Pelizaeus-Merzbacher disease}

Duplication of X-linked plp1 gene (proteolipid protein gene 1) which results in overexpression of PLP protein is the most common cause of Pelizaeus-Merzbacher disease (PMD), a fatal leukodystrophy without therapeutic options (Seitelberger 1995). PLP overexpressing transgenic (PLP1 tg) mice is the mouse model of this disease (PMD mice). The main function of PLP is to maintain the integrity of 
myelin structure(Duncan, Hammang et al. 1987). PLP overexpressing transgenic mouse is considered as a non-immune mediated demyelination model in which dysmyelination starts at the optic nerve and spread into the brain within several months (Edgar, McCulloch et al. 2010) Oligodendrocyte death and axonal degeneration are other characteristics of this mouse model (Anderson, Schneider et al. 1998).

\subsubsection{Microglia activation in PLP overexpressing transgenic mice}

Activated microglia are found in white and gray matter very early on the $7^{\text {th }}$ postnatal day, and by the third postnatal week microglia cells are endogenously reproduced. Microglia produce a high level of TNF- $\alpha$ and IL- 6 in this stage; in addition, CC chemokines ligands and receptors including CCL3, 4, 8, CCR1 and 6, and also CXCL9 and 10 are up-regulated. Since activated microglia are found when the myelination has not started even in normal condition, it has been then suggested that myelin degradation does not trigger microglia activation in $p / p 1 \mathrm{tg}$ mice. Overexpression of PLP, but not myelin abnormalities, is thought to be the reason for the primary immune activation.

In PLP overexpressing transgenic mice, microglia-induced inflammation is considered to be important for axonal lost and disease progress (Bradl, Bauer et al. 1999; Tatar, Appikatla et al. 2010). In addition, short and thick processes are the common morphological features of activated microglia cells that are detectable in both white and gray matter when the mice are at 21 days of age. However, these changes are not seen in all microglia; for example, in the striatum and corpus callosum the processes are longer as they are in normal condition (Tatar, Appikatla et al. 2010).

\subsection{Endocytic pathway}

\subsubsection{Endosome biogenesis}

Lysosomes are catabolic compartment in eukaryotic cells, important for degradation of internalized materials and also components which are processed by autophagy (Kornfeld and Mellman 1989; Funato, Beron et al. 1997). Many different proteins are involved in endocytic trafficking regulating endosome maturation, movement, fission, and fusion. Small molecular weight G-proteins called Rab GTPase play a major role in endocytic pathway (Zerial and McBride 2001). These proteins belong to the Ras suprfamily of GTPases, they are located in the cytosolic part of intracellular membranes, and regulate different steps in membrane traffic pathways by switching between active (GTP-bound) and inactive (GDP-bound) forms. In this context, Rab proteins facilitate vesicle motility, tethering, and 


\section{1 | Introduction}

fusion (Stenmark and Olkkonen 2001; Pfeffer and Aivazian 2004; Gurkan, Lapp et al. 2005). 11 and 60 Rab proteins have been identified in yeast and human, respectively (Pereira-Leal and Seabra 2001). Rab4a, Rab5a, Rab5b, Rab5c, Rab11, Rab18, Rab22, and Rab25 are localized in the early endosomal compartments (Bucci, Parton et al. 1992; Lutcke, Parton et al. 1994). Rab7 and Rab9 are found in the late endosomal compartments (Chavrier, Parton et al. 1990; Lombardi, Soldati et al. 1993). Rab5 is responsible for the formation of early endosome by regulating the fusion of endocytic vesicles (Hutagalung and Novick 2011). When cargo is trafficked through endocytic compartments, Rab5 is replaced by Rab7; this switch between Rab5 and 7 mediates transformation of early to late endosome (Rink, Ghigo et al. 2005; Vonderheit and Helenius 2005).

\subsubsection{Lysosome biogenesis, Role of Rab7}

Rab7 is involved in endocytic trafficking, specifically, in trafficking of early and late endosome; Rab7 regulates the movement of cargo out of early endosomes to late endosome (Chavrier, Parton et al. 1990; Vitelli, Santillo et al. 1997; Vonderheit and Helenius 2005). Rab7 has also been found to be required for the fusion between late endosome and lysosome. Its function is also important for cargo transfer from late endosome to lysosome (Bucci, Thomsen et al. 2000; Vanlandingham and Ceresa 2009). Moreover, by expressing Enhanced Green Fluorescent Protein (EGFP)-tagged wild type Rab7 and also using dominant-negative mutant proteins it has been shown that Rab7 is required for the biogenesis and maintenance of lysosomal structure. In the absence of Rab7, the lysosomes cannot be aggregated around the nucleus so that they are diffused throughout the cytoplasm. Therefore, it has been suggested that Rab7 might regulate the movement of vesicles along the cytoskeleton by engaging motor proteins such as myosin I, kinesin, and dynein to facilitate the aggregation of lysosomes around the nucleus. In addition, Rab7 controls the fusion of late endocytic compartments by regulating tight membrane interactions. Furthermore, acidification of lysosomes, which is necessary for the activity of hydrolases, the main lysosomal enzymes,(Mellman, Fuchs et al. 1986) is disturbed in the absence of Rab7. In conclusion, Rab7 is required for function of late endocytic structures including endosomes and lysosomes in the perinuclear region (Bucci, Thomsen et al. 2000) (Fig 1.3). 


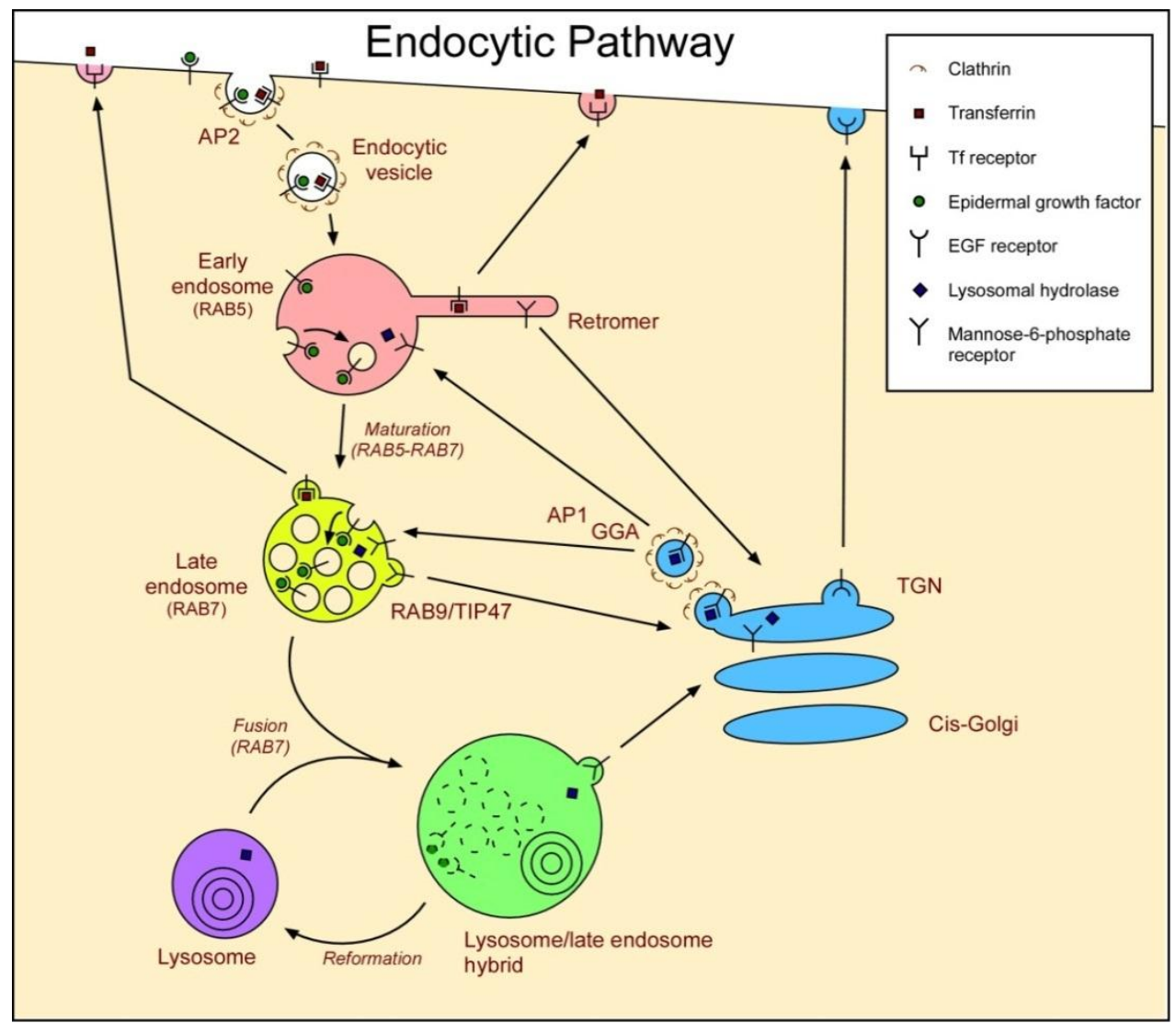

Figure 1.3 Rab proteins involved in endocytic pathway. Rab5 mediates the fusion of early endososmes, and the exchange between Rab5 and Rab7 leads to the transformation of early endososme to the late compartment. Rab7 is involved in downstream endocytic pathway, required for cargo transfer from early endosome to late endosome as well as for the fusion between late endosome and lysosome. Cargo transfer from endosomes to the Golgi complex is also carried out by Rab proteins, such as Rab9. Reprint from Wikipedia, Created by Matthew R G Russell, license: CC BY-SA 3.0.

\subsection{Question and hypothesis of the project}

The main question of our project was how myelin molecules that are trapped within the numerous layers of tightly compacted membrane get access to the degradation system for myelin turnover. We hypothesized that myelin fragments which are formed by shedding myelin sheath in the extracellular space may be engulfed by microglia cell. Furthermore, we asked how the possible interaction of microglia with myelin affects the cell function with time. We hypothesized that overloading lysosomes with myelin components might induce senescence phenotype in microglia. 


\section{Chapter 2}

\section{Materials and Methods}

\subsection{Materials}

\subsubsection{Antibodies}

Primary antibodies:

Table 2. 1 Primary antibodies list

\begin{tabular}{ccccc}
\hline Targen antigen & Host species & Clone & Dilution & Company \\
\hline Iba1 & Rabbit & - & $1: 1000$ & Wako \\
MBP & Mouse & SMI94 & $1: 1000$ & Covance \\
MBP & Rabbit & - & $1: 500$ & Dako \\
Mac2 (Galectin 3) & Rat & M3/38 & $1: 200$ & Biolegend \\
MHCII & Rat & M5/114.15.2 & $1: 200$ & eBioscience \\
LAMP1 & Rat & $1 D 48$ & $1: 200$ & Santa Cruz \\
CR3/Mac1/CD11b & Rat & - & $1: 200$ & Biotechnology \\
Mac3 & Rat & M3/84 & $1: 200$ & BD Pharmingen \\
CD45 & Rat & - & $1: 200$ & Bio-Rad \\
Fcy RII/III/ CD16/32 & Rat & $2.4 G 2$ & $1: 200$ & BD Pharmingen \\
\hline
\end{tabular}

\section{Secondary antibodies:}

All biotinylated secondary antibodies (IgG) for $D A B$ staining were purchased from Vector Laboratories. Fluorescence secondary antibodies including Alexa Fluor 488, 555, 647, Cy3 and Cy5 were purchased from Dianova and Invitrogen. Streptavidin-conjugated Alexa Fluor 488 was from Invitrogen. Anti-mouse and anti-rabbit horse radish peroxidase (HRP)-conjugated secondary antibodies used for Western blot were from Dianova. 


\subsubsection{Commercial kits}

Table 2.2 List of commercial kits

\begin{tabular}{lll}
\hline \multicolumn{1}{c}{ Kit } & \multicolumn{1}{c}{ Application } & \multicolumn{1}{c}{ Company } \\
\hline Spin Tissue Midi Kit & DNA extraction for genotyping & Invitek \\
NucleoSpin RNA XS & total RNA isolation & Macherey-Nagel GmbH \& Co.KG \\
Neural Tissue Dissociation Kits & Microglia isolation & Miltenyi Biotec \\
\hline
\end{tabular}

\subsubsection{Primers}

All the primers were synthesized by ACGTLab, DNA core facility at Max Planck Institute for Experimental Medicine, they are listed as following:

Table 2.3 list of primers

\begin{tabular}{|c|c|c|}
\hline Primer ID & Sequence & description \\
\hline 25174 & 5'-CAC GGG GGA GGC AGA GGG TTT-3' & Fwd for E2A Cre; Neo \\
\hline 25175 & 5'-GCG GAG CAC GGG CCA CAT TTC-3' & Rev for E2A Cre; Neo \\
\hline 26230 & 5'-AGATGACGTAGTTTTCGCGCTT-3' & Fwd for Ella promotor \\
\hline 17411 & 5'-TCCGGTTATTCAACTTGCAC-3' & Rev for ParvCRE \\
\hline 24050 & 5'-TATCTTCTATATCTTCAGGCGC-3' & Fwd for CX3CR1 Cre ERT2 \\
\hline 24051 & 5'-GTGAACGAACCTGGTCGAAATCAG-3' & Rev for CX3CR1 Cre ERT2 \\
\hline 24052 & 5'-CTCACTCACTCCTAAATGG-3' & for wild type Rab7 allele \\
\hline 24053 & 5'-TTAGGCTGTATGTATGTGC-3' & for Floxed Rab7 allele \\
\hline 21027 & 5'-TACGGCATGGACGAGCTGTACAAGTAA-3' & Fwd tdTomato \\
\hline 21028 & 5'-CAGGCGAGCAGCCAAGGAAA-3' & Rev tdTomato \\
\hline 29421 & 5'-GGAATCGGACGTCTCTGTTG-3' & Fwd for amplification of Rab7 cDNA \\
\hline 29422 & 5'-AGTCCCCCAGGATGATGAC-3' & Rev for amplification of Rab7 cDNA \\
\hline 1879 & 5'-ATGTATGTGTGTGTGTGCTTATCTAGTGTA -3' & Shiverer-Fwd \\
\hline 1880 & 5'-CAGGGGATGGGGAGTCAGAAGTGAG -3' & Shiverer-Rev \\
\hline 1873 & 5'-CCCCGTGGTAGGAATATTACATAAC -3' & Wild type for shiverer detection-Fwd \\
\hline 1874 & 5'-AGCTCTGGTCTTTCTTGCAG -3' & Wild type for shiverer detection-Rev \\
\hline
\end{tabular}




\subsubsection{Software}

In this study the following software were used for primer design, data acquisition, and image processing and analysis.

Table 2.4 List of Softwares

\begin{tabular}{lll}
\hline software & application & source \\
\hline Imag J & Image processing & http://imagej.nih.gov/ij/ \\
Imaris & Image processing and analysis & Bitplane \\
Adobe Illustrator CS4 & Image processing & Adob System Inc. \\
ApE & DNA editing and analysis & Wayne Davis, University of Utah \\
GrafPad Prism & Data analysis, plot generation, and & http://www.graphpad.com/scientific- \\
EndNote & statistics & software/prism/ \\
Leica Confocal & Bibliography management & Thomas Reuters \\
Software & Confocal images acquisition & Leica Microsystems, Mannheim, Germany
\end{tabular}

\subsubsection{Solutions and Media}

In this study some solutions or media were commercially available, and some were manually prepared as described below:

Table 2.5 Commercial components and media

\begin{tabular}{ll}
\hline Media & Copmany \\
\hline DMEM for Primary cell culture & Gibco/Invitrogen \\
Fetal Calf Serum (FCS) & PAA \\
GlutaMAX'M-I supplement & Gibco/Invitrogen \\
Penicillin/Streptomycin (Pen/Strep) 100 & Gibco/Invitrogen \\
Protease inhibitors cocktail & Sigma \\
1X PBS & Gibco/Invitrogen \\
1X HBSS & Gibco/Invitrogen \\
Power SYBR Green PCR master mix (2X) & Invitrogen \\
Trypsin & Invitrogen \\
\hline
\end{tabular}

10X PBS (phosphate buffered saline):

$80 \mathrm{~g} \mathrm{NaCl}$

$2 \mathrm{~g} \quad \mathrm{KCl}$

$18.05 \mathrm{~g} \mathrm{Na2HPO}$ _ 2 $2 \mathrm{H} 2 \mathrm{O})$

$2.4 \mathrm{~g} \quad \mathrm{KH} 2 \mathrm{PO} 4$

Dissolved in $1 \mathrm{~L}$ distilled $\mathrm{H}_{2} \mathrm{O}$. PH was adjusted to 7.4. 


\subsubsection{Mouse lines}

The following mouse lines were used and analyzed in this study:

Table 2.6 List of mouse line

\begin{tabular}{ll}
\hline Strain name & source \\
\hline \multirow{2}{*}{ Rab7 flox/flox } & Aimee Edinger, Department of Cell \& Developmental Biology University of California, \\
CX3CR1 ${ }^{\text {CreERT2 }}$ & Irvine \\
tdTomato reporter & Dr. Steffen Jung, Department of Immunology, Weizmann Institute of Science \\
Ella Cre (E2A Cre) & The Jackson Laboratory, Stock Number: 007905 \\
Shiverer & The Jackson Laboratory, Stock number: 003724 \\
Rac1 flox/flox & --- \\
Plp1-overexpressing transgenic mice & The Jackson Laboratory, Stock number: 005550 \\
& Klaus-Armin Nave, Department of Neurogenetics, \\
CX3CR1 GPP/+ & Max Planck Institute of Experimental Medicine, Göttingen, Germany. \\
& Klaus-Armin Nave, Department of Neurogenetics, \\
& Max Planck Institute of Experimental Medicine, Göttingen, Germany. \\
\hline
\end{tabular}

\subsection{Methods}

\subsubsection{Generation and characterization of conditional Rab7 KO mice}

\subsubsection{Generation and breeding of mutant mice}

In order to block lysosomal degradation system in microglia, we took advantage of Cre-lox recombination system and the following strategy was performed. A mouse line carrying Rab7 allele flanked by loxP sites (Rab7 $7^{\text {flox/flox }}$, from Dr. Aimee Edinger, Department of Cell \& Developmental Biology University of California, Irvine), and the mice carrying a tamoxifen inducible Cre recombinase (Cre-ERT2) in CX3CR1 locus (from Dr. Steffen Jung, Department of Immunology, Weizmann Institute of Science) were used. In the latter mouse line the expression of Cre recombinase is derived by CX3CR1 promoter which is specifically expressed in microglia. In these mice the Cre-ERT2 along with a loxP-flanked neomycin resistance cassette replaces CX3CR1 exon 2 (Fig 2.1). For details of targeting and deleted region in the CX3CR1 locus see (Jung, Aliberti et al. 2000). To remove the Neomycin resistance cassette from CX3CR1 ${ }^{\text {CreERT2-neo }}$ locus through Cre-mediated excision, the mice carrying Cre ERT2-Neo were crossed to Ella-Cre (E2A Cre) mouse line which expresses Cre recombinase from 
oozyte stage onwards, and in all cell types (Deleter-Cre) (The Jackson Laboratory, B6.FVB-Tg(Ella-cre) C5379Lmgd/J, Stock number: 003724). In the obtained chimeric mice (CX3CR1 ${ }^{+/ \text {CreeRT2-neo }}$ Ella ${ }^{+/ \text {Cre }}$ ), due to Cre recombination mediated by Ella-Cre, the floxed neomycin cassette was excised but not with $100 \%$ efficiency. Therefore, removal of neo cassette was confirmed by doing a PCR using following primer pair, 5'-CACGGGGGAGGCAGAGGGTTT-3'; 5'-GCG GAGCACGGGCCACATTTC-3' which results in amplification of a $500 \mathrm{bp}$ fragment indicative of $\mathrm{CX} 3 \mathrm{CR} 1^{+/ \text {CreERT2 }}$ locus without neo cassette, and a 1800 bp product specific for CX3CR1 ${ }^{+/ \text {CreERT2 }}$ locus with neo cassette. To remove Ella-Cre locus from the chimeric mice with excised neo cassette, these mice were mated with C57BL/6J wild type mice. PCR reactions were done using two set of primers as follows: sense Ella promoter (P1): 5'AGATGACGTAGTTTTCGCGCTT-3'; antisense Cre (P2): 5'-TCCGGTTATTCAACTTGCAC-3', and P3: 5'TATCTTCTATATCTTCAGGCGC-3'; P4: 5'-GTGAACGAACCTGGTCGAAATCAG-3'. The combination of P1 and P2 amplified a 387bp product specific for Ella ${ }^{\text {Cre }}$ locus, and the mix of P3 and P4 produced a $223 b p$ fragment for $\mathrm{CX} 3 \mathrm{CR} 1^{\text {Cre ERT2 }}$ locus. In this step, we got CX3CR1 $1^{+/ \text {CreeRT2 }}$ mice.

To obtain double transgenic mice (CX3CR1 $1^{+/ \text {CreeRT2 }}$; Rab7 $7^{\text {flox/flox }}$ )two sets of breeding was arranged with $\mathrm{CX} 3 \mathrm{CR} 1^{+/ \mathrm{CreERT2}}$ and $\mathrm{Rab}^{\text {flox/flox }}$ mice. For genotyping the offspring of these breeding sets two distinct PCR reactions using the combination of P3 and P4 and the following primer pair were done: wild type Rab7 allele: 5'-CTCACTCACTCCTAAATGG-3'; Floxed Rab7 allele: 5'TTAGGCTGTATGTATGTGC-3'. PCR products amplified by the latter primer pair were a 550 bp band specific for wild-type Rab7 and a 580 bp band representing floxed Rab7 allele (2 Lox: floxed allele without the Neo cassette).

Targeting vector

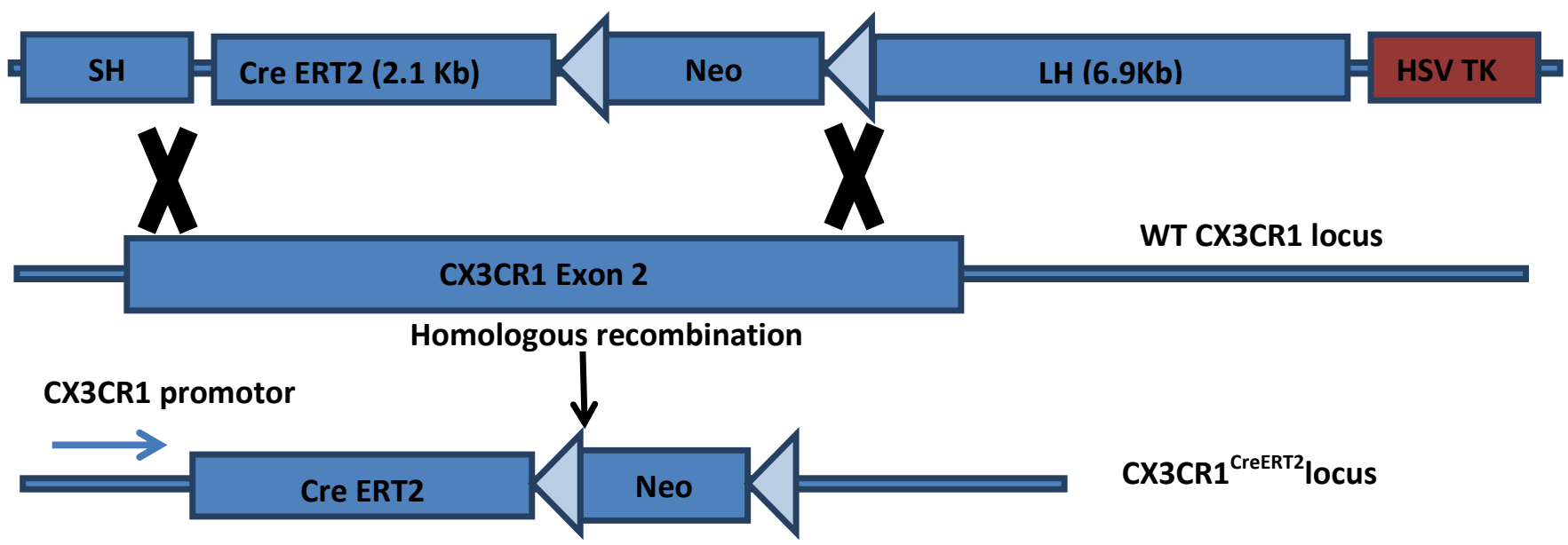

Figure 2.1 Targeted disruption of the CX3CR1 locus in mouse 


\subsubsection{Tamoxifen induction of conditional deletion of Rab7 gene}

Double transgenic mice (CX3CR1 $1^{+/ \text {CreERT2 }}$; Rab $7^{\text {flox/flox }}$ ) were injected with tamoxifen at 3 weeks of age ,as described below, to induce conditional deletion of Rab7 in microglia of these mice and obtain conditional Rab7 knockout mice. Control mice of the same genotype received corn oil vehicle only.

\section{Tamoxifen preparation and administration}

The animal treatment with tamxifen was approved in advance by the Lower Saxony state authorities ("Niedersächsisches Landesamt für Verbraucherschutz und Lebensmittelsicherheit"; Postfach 39 49; 26029 Oldenburg). The Approval ID is: 33.14.-42502-04-117/09. Tamoxifen (Sigma T5648) was freshly prepared the day before injection. It was dissolved in filter-sterilized corn oil to make solution of 10 $\mathrm{mg} / \mathrm{ml}$. The solution was protected from light, and placed on the roller mixer to be dissolved over night at $37^{\circ} \mathrm{C}$. Tamoxifen was administrated via intraperitoneal injection once every 24 hours for a total of 5 consecutive days. The injection dose was determined by weight, using approximately $75 \mathrm{mg}$ tamoxifen/kg body weight. For adult mice, a standard dose of $100 \mu$ tamoxifen/corn oil solution was effective to induce cre recombinase activity.

\subsubsection{Detection of Cre recombinase activity using reporter mice}

To test the activity of Cre recombinase in microglia, CX3CR1 ${ }^{+/ C \text { reERT2 }} ;$ Rab7 $7^{\text {flox/flox }}$ mice were crossed to reporter mice carrying tdTomato gene which is located downstream of a loxP-flanked STOP cassette. The offspring of this breeding, CX3CR1 $1^{+/ C r e E R T 2}$; Rab ${ }^{\text {flox/+ }}$; tdTomato+, was injected with Tamoxifen at 3 weeks of age as the same procedure described previously to drive the translocation of Cre recombinase to the nucleus, where it is able to delete the floxed STOP cassette, and result in tdTomato expression. One week after Tamoxifen injection the expression of tdTomato gene in microglia was confirmed by immunohistochemistry.

\subsubsection{Cre recombinase-mediated deletion of Rab7 gene in microglia}

To confirm deletion of Rab7 gene mediated by CX3CR1-driven expression of Cre recombinase, one and six weeks after Tamoxifen injection microglia cells from double transgenic mice (CX3CR1 ${ }^{+/ \text {CreERT2 }}$; $\left.R a b 7^{f l o x / f l o x}\right)$ were isolated as will be explained in section 2.2.6.1. The total RNA was isolated from 
cells; cDNA was synthesized, and Rab7 gene expression was quantified using real-time PCR as will be described later.

\subsubsection{Genotyping}

The genomic DNA was extracted from the lysate of a small piece of mouse tail using the Invitek Spin Tissue Kit according to the manufacturer's instruction. The DNA was eluted in $100 \mu l$ elution buffer, and the concentration of DNA was defined by measuring the absorbance of double stranded DNA at 260nm with a BioPhotometer plus spectrophotometer (Eppendorf). 0.1-1 $\mu$ g of DNA was amplified by GoTaq DNA polymerase (Promega, Mannheim, Germany).

\section{PCR master mix for a $25 \mu$ reaction was prepared as following:}

$15.35 \mu \mathrm{l}$ milli Q $\mathrm{H} 20$

$5 \mu l 5 X$ Taq buffer

$0.5 \mu \mathrm{l}$ dNTPs $(10 \mathrm{mM}$, stock)

$0.5-1 \mu \mathrm{l} \mathrm{MgCl} 2$ (25mM, stock) (The final concentration depends on the primers)

$1 \mu \mathrm{l}$ forward primer $(10 \mathrm{pmol} / \mu \mathrm{l}$, stock)

$1 \mu \mathrm{l}$ reverse primer $(10 \mathrm{pmol} / \mu \mathrm{l}$, stock)

$0.15 \mu \mathrm{l}$ Taq DNA Polymerase (5 units/ $\mu \mathrm{l})$

$1 \mu \mathrm{I}$ DNA $(0.1-1 \mu \mathrm{g})$

The PCR was performed using a T3000 Thermocycler Kombi (Biometra) as following:

\begin{tabular}{llll} 
Step & Temperature $\left({ }^{\circ} \mathrm{C}\right)$ & Time & Number of cycles \\
\hline Initial denature & 94 & 3 minutes & 1 \\
\hline Denature & 94 & 45 Seconds & \\
Annealing & Depending on the primers & 45 Seconds & $30-35$ \\
Extension & 72 & 1 minute & \\
\hline Final extension & 72 & 5 minutes & 1
\end{tabular}




\section{Agarose gel electrophoresis}

1\% Agarose gel in 1X TAE buffer containing 1X SYBR safe DNA gel stain (10000X, stock, Invitrogen) was prepared. Electrophoresis was done in the Chambers assembled by the Feinmechanik Service Department at the Max Planck Institute for Experimental Medicine containing 1 X TAE buffer. The PCR products were visualized by transillumination using Intas Gel Documentation System (Intas Science Imaging Instruments $\mathrm{GmbH}$ ).

\section{X TAE (1 L):}

48.4 $\mathrm{g}$ Tris base

$11.4 \mathrm{~mL}$ Acetic acid

$20.0 \mathrm{~mL} 0.5 \mathrm{M}$ EDTA

Adjust to $1 \mathrm{~L}$ with ddH2O, $\mathrm{pH} 8.5$

\subsubsection{Real-time quantitative PCR}

\section{RNA Isolation}

Total RNA was extracted from isolated microglia using the Nucleospin RAN XS kit according to the manufacturer's instruction, the concentration was obtained by measuring the absorbance of RNA at 260nm using a BioPhotometer plus spectrophotometer (Eppendorf).

\section{Reverse transcription}

Reverse Transcription of RNA into cDNA was performed using SuperScript ${ }^{\circledR}$ III First-Strand Synthesis System. The RNA/Primer mixture is as following:

\begin{tabular}{ll}
\hline Component & Amount \\
\hline $0.2 \mu \mathrm{g}$ total RNA & $\mathrm{n} \mu \mathrm{l}$ \\
$50 \mu \mathrm{M}$ oligo (dT) & $1 \mu \mathrm{l}$ \\
$10 \mathrm{mM}$ dNTP mix & $1 \mu \mathrm{l}$ \\
DEPC-treated water & To $10 \mu \mathrm{l}$ \\
\hline
\end{tabular}


The mixture was incubated at $65^{\circ} \mathrm{C}$ for 5 minutes then placed on ice at least for one minute. Meanwhile, cDNA Synthesis Mix was prepared as following:

\begin{tabular}{ll}
\hline Component & Amount \\
\hline 10X RT buffer & $2 \mu \mathrm{l}$ \\
$25 \mathrm{mM} \mathrm{MgCl}_{2}$ & $4 \mu \mathrm{l}$ \\
$0.1 \mathrm{M}$ DTT & $2 \mu \mathrm{l}$ \\
RNaseOUT $(40 \mathrm{U} / \mu \mathrm{l})$ & $1 \mu \mathrm{l}$ \\
SuperScript III RT $(200 \mathrm{U} / \mu \mathrm{l})$ & $1 \mu \mathrm{l}$ \\
\hline
\end{tabular}

Afterwards, $10 \mu \mathrm{l}$ of cDNA Synthesis Mix was added to RNA/Primer mixture, incubated at $50^{\circ} \mathrm{C}$ for 50 minutes, the reaction was terminated at $85^{\circ} \mathrm{C}$ for 5 minutes, chilled on ice. The residual RNA was digested by Riboneuclease $\mathrm{H}$ for 20 minutes at $37^{\circ} \mathrm{C}$. cDNA was stored at $-20^{\circ} \mathrm{C}$ for further use.

\section{Real-time quantitative PCR}

Expression levels of Rab7 in microglia isolated from adult mouse brain were measured by relative reverse transcriptase quantitative real-time PCR (RT-qPCR). The primers were designed with NCBI Primer Blast software. For each condition triplicates were used. The expression of the target gene was measured in relation to internal levels of GAPDH and 18srRNA as reference genes. The quantitative PCR was performed using SYBR Green PCR Master Mix according to manufacturer's protocols. The relative change in gene expression was analyzed by $\Delta \mathrm{Ct}$ method, and normalized to the control samples.

\section{Master mix composition:}

\begin{tabular}{ll} 
Component & Amount \\
\hline 2X SYBR Green Master Mix & $5 \mu \mathrm{l}$ \\
Primer-Forward $(50 \mathrm{pmol} / \mu \mathrm{l})$ & $0.2 \mu \mathrm{l}$ \\
Primer-Reverse $(50 \mathrm{pmol} / \mu \mathrm{l})$ & $0.2 \mu \mathrm{l}$ \\
Nuclease-free $\mathrm{H}_{2} \mathrm{O}$ & $1 \mu \mathrm{l}$
\end{tabular}


Following adding 4 microliter of cDNA (20ng), $6 \mu$ l of master mix was added into the each well of 96well plate. PCR was done in a light cycler 480.

\subsubsection{Histology}

\subsubsection{Perfusion and tissue preparation}

Animals were anesthetized by intraperitoneal injection of $14 \%$ chloral hydrate, perfused transcardially with $4 \%$ parafolmaldehyde (PFA) using a MPII mini peristaltic pump (flow rate: $3 \mathrm{ml} / \mathrm{min})$. After perfusion, brain tissue was post fixed in 4\% PFA overnight. Then the tissue was cryoprotected in $30 \%$ sucrose in $1 \times$ PBS until when the brain sank (at least one day). The tissue was bound to the specimen block using Tissue-Tek O.C.T, frozen on dry ice, and wrapped in pre-cold aluminum foil. Free floating cryosections ( $30 \mu \mathrm{m}$ thickness) were obtained in coronal direction using cryostat Leica CM 1900, placed in 48-well plates containing cryopreservative solution (25\% ethylenglycol, and $25 \%$ glycerol in $1 \times$ PBS), and stored at $-20^{\circ} \mathrm{C}$.

\subsubsection{Immunohistochemistry}

The following antibodies were used for immunohistochemistry:

Primary antibodies: Iba1 (lonized calcium binding adapter molecule 1) against ramified microglia, Mac2 (Galectin 3) and MHCII (major histocompatibility complex class II), Mac3, CD11b (Complement receptor 3 or Mac1), CD16/32 (Fcy receptor II/III), CD45 for activated microglia, MBP (Myelin Basic Protein) against myelin, LAMP1 (lysosomal-associated membrane protein 1) for lysosomes (For more details see table 2.1).

Secondary antibodies: For DAB staining we used goat anti rat biotinylated immunoglobulin G (1:200) and for fluorescent staining Alexa Flour 488, 647, and 555-conjugated antibodies were used (1:1000).

\section{Immunohistochemistry with Fluorescence detection}

Free floating sections were rinsed with $1 x$ PBS containing $0.2 \%$ Tween-20 in 24-well plate. Permeabilization was performed in $0,5 \%$ Triton $X-100$, the incubation time varied from 10 to 30 minutes depending on primary antibody. For blocking endogenous mouse tissue immunoglobulins, fab fragment goat anti mouse IgG (1:100) (dianova) was added for 1 hour at RT. The sections were washed briefly and incubated in $100 \%$ blocking solution (2.5\% FCS, $2.5 \% \mathrm{BSA}, 2.5 \%$ fish gelatin in $1 \mathrm{x}$ PBS) for 1 hour at RT. Primary antibodies, diluted in 10\% blocking solution, were added and incubated overnight at $4^{\circ} \mathrm{C}$. On the following day, after washing the sections with PBS 3 time each 10 
minutes, they were incubated in secondary antibodies, diluted in $10 \%$ blocking solution, for 1 hour at RT. The sections were washed with PBS, distilled $\mathrm{H}_{2} \mathrm{O}$ and mounted using fluorescence mounting medium (Dako) over superfrost plus slides.

\section{Immunohistochemistry with HRP-DAB detection}

3,3-diaminobenzidine (DAB) immunohistostaining was performed using VECTASTAIN ${ }^{\circledR}$ ABC Kit standard as following: Free floating cryosections were transferred to a 24-well plate, washed three times with PBS, each time 5 minutes on the shaker. Endogenous peroxidase was blocked with 3\% $\mathrm{H}_{2} \mathrm{O}_{2}$, incubated for 20 minutes at $4^{\circ} \mathrm{C}$, and washed out with PBS. Then $100 \%$ blocking solution (as before) was added to the sections, incubated for 20 minutes at room temperature (RT). The sections were incubated with primary antibodies, diluted in $10 \%$ blocking solution, overnight at $4^{\circ} \mathrm{C}$. Afterwards, the sections were washed with PBS, incubated with biotinylated secondary antibodies

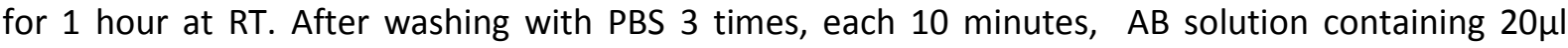
reagent A (Avidin DH) and $20 \mu$ l reagent B (Biotinylated Horseradish Peroxidase H) in 1x PBS was added to the sections and incubated for 30 minutes at RT. Following three washes, (each 5 minutes) staining was developed using 3,3'-diaminobenzidine (DAB) staining kit (Vectastain, Vector Laboratories). The incubation time varied depending on primary antibody. DAB reaction was stopped with distilled water for 5 minutes on the shaker. Then sections were washed with PBS, and placed on super frost plus microscope slides to let them dry for 2-3 days. The sections were rehydrated in decreasing concentrations of ethanol, and finally in water, stained with hematoxilin for 1 minute, and dehydrated using increasing percentages of alcohol, cleared in xylol (Chemie Vertrieb $\mathrm{GmbH}$ ) and mounted with DePeX.

\subsubsection{Lipofuscin autofluorescent reduction}

\subsubsection{Chemical treatment}

To reduce lipofuscin autofluorescent in old brain tissues after immunohistochemistry, the sections were removed from PBS, dipped briefly in distilled water, and treated with 10mM CuSO4 (Fisher

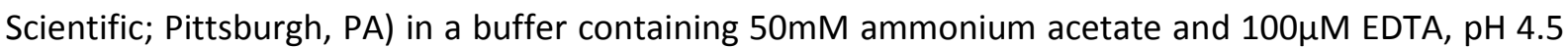
for 90 min on a shaker. The sections were washed briefly in distilled water, and transferred to PBS. (Schnell, Staines et al. 1999) 


\subsubsection{Dye separation}

Autofuorescent of lipofuscin was a significant problem in fluorescent microscopy. Therefore, by means of dye separation tool on Leica SP5 Confocal microscope the autofluorescent was treated as another fluorophore so that it could be removed from the specific signal. An unstained sample as a reference was used then the emission spectrum of lipofuscin was determined using lambda scanning option on Leica SP5 confocal microscope. The obtained spectrum should be saved in spectra database.

To separate lipofuscin spectrum from a labeled sample (either with Alexa Flour 488 or 555) "spectral dye separation tool" was chosen, a region of interest (ROI) was manually drawn, the size of reference region was 10 in voxels. The measured emission spectrum which was displayed in a dialog box was added to spectra database; it was then available in the list under user. Afterwards, in the spectral dye separation dialog, lipofuscin spectrum was selected from the database, and by selecting "All channels" in "rescale box" all channels were rescaled together using the same factor, finally by applying all these factors the image was processed to get an image without crosstalk with lipofuscin spectrum.

\subsubsection{Imaging}

\subsubsection{Confocal microscopy}

In this study, laser scanning microscopy was used for image acquisition because confocal microscopy provides higher resolution images with less background than conventional light microscopy. Confocal microscopic images were taken using a Leica TCS SP5 AOBS confocal laser scanning setup (Leica Microsystems, Mannheim, Germany).

\subsubsection{Electron microscopy}

The mouse brain was fixed by transcardial perfusion using $4 \%$ paraformaldehyde and $2.5 \%$ glutaraldehyde in $0.1 \mathrm{M}$ phosphate buffer containing $0.5 \% \mathrm{NaCl}$. The brain was extracted, post fixed in the same fixative solution overnight. The tissue was sectioned into 200 micron thick vibrotome sections. Rostral and caudal regions of corpus callosum was cut and post-fixed in a solution of $1 \%$ osmium tetroxide in $0.1 \mathrm{M}$ phosphate buffer $(\mathrm{pH} 7.4)$ for 30 minutes at room temperature. Following washing with distilled water the sections were stained with $0.5 \%$ uranyl acetate in $70 \%$ ethanol for one hour, dehydrated in a serial dilution of ethanol, and cleared in propylene oxide and embedded in Epon, incubated at $60^{\circ} \mathrm{C}$ for 24 hours. The tissues in Epon blocks was then trimmed and reoriented so 
that ultrathin $(60 \mathrm{~nm})$ cross section of midline corpus callosum could be cut using ultramicrotome. Ultrathin sections were collected on collodion-coated copper grids. The sample area was selected at low magnification of $2500 x$.

\subsubsection{In vivo time-lapse imaging}

Mice were initially anesthetized by an intraperitoneal (i.p.) injection of a mixture of dorbene (Pfizer), ketamine (Medistar) in $0.9 \% \mathrm{NaCl}$ to achieve a dose of $1 \mathrm{mg} / \mathrm{kg}$ body weight for dorbene and 100 $\mathrm{mg} / \mathrm{kg}$ for ketamine. The surgical plane of anesthesia was monitored by paw pinch reflex. Typically, half of the initial dose was injected i.p. every 60 min during imaging session according to the level of anesthesia. The eyes were protected from drying out with Bepanthen (Bayer). The hair of head was shaved with a small animal clipper (Pocket Pro, Wahl). Afterwards, Mice were placed on the custombuild microscope stage and the temperature was maintained at $36-37{ }^{\circ} \mathrm{C}$ using a built-in heating block (Telemeter Electronic GmbH, Donauwörth, Germany). Using a rectal temperature probe (Telemeter Electronic $\mathrm{GmbH}$, Donauwörth, Germany) the body temperature was monitored during imaging process. To control the breath of animal, an oxygen stream with the controlled pressure was transferred to a mask which was positioned in front of the snout. The surgery got started with removing the skin on top of skull and cleaning skull so that it became free of hair and connective tissue and dry. Then a custom-built ring is fixed on the skull bone using Durelon ${ }^{\mathrm{TM}}$ Carboxylate Luting Cement (3M ESPE). Once the cement is completely dry and stably fixed in the setup, the bone in the center of the ring is thinned using a dental micromotor (FOREDOM ${ }^{\circledR}$ Electric Co.). The ring around the thinned area provides a waterproofed pool around the area of interest for imaging; the ring was filled with $0.9 \% \mathrm{NaCl}$. To acquire the images, a Laser Scanning Microscope 710 from Carl Zeiss equipped with a 20x Plan-APOCHROMAT water immersion objective with a high numeric aperture (NA 0.95) was used. A modelocked Ti: sapphire Chameleon Vision II laser system from Coherent was adjusted to $880 \mathrm{~nm}$ wavelength. Excited fluorescence was detected with nondescanned detectors endowed with 525/45-nm and 624/40-nm band-pass filters (Semrock, Inc.). The live imaging was done for 15 minutes.

Image analysis: To quantify the dynamics of the cell process motility, time lapse movies were analyzed with Imaris software as following. A single process of the cells was segmented; a surface was created for the whole area and volume of the process. The surface with the same threshold was applied for the same process in the following images every 32 seconds. Using the surface, the area occupied by the process was measured automatically every recording period of 32 seconds. Two processes of 5 cells were analyzed. Finally, variance between the sizes of process area, which was obtained every 32 seconds, was calculated and compared between different groups. 


\subsubsection{Image processing and analysis}

In this project all the Images were processed and analyzed with Imaris (64x version 7.7.1) and Image J 1.41 image processing software.

\subsubsection{Cell counting}

To estimate the number of Iba1 positive cells, confocal stacks (step size: $0.8 \mu \mathrm{m}$ ) were captured in the z-direction from the whole region of interest with 20X or 40X objectives of a Leica TCS SP5 confocal microscope.

An area in the size of $1 \mathrm{~mm}^{2}$ in the region of interest was selected, the total number of Iba1 positive cell bodies and as well as the number of Iba1 positive cells with internalized components (such as FITC-Dextran or MBP or lipofuscin) were counted using cell counter plugin in ImageJ. In addition, to confirm the quantification performed by ImageJ, cell counting was done automatically using Imaris software. Briefly, a region of interest was segmented and spots layer was created (radius scale: 8 ) for each marker (Iba1 and FITC/MBP/LF) in the corresponding channel, using those spots the cells were counted automatically. The colocalized spots were defined in the distance of $0.2 \mu \mathrm{m}$ (threshold value).

\subsubsection{Lipofuscin volume measurement}

To measure the size of lipofuscin within microglia, the individual cells were analyzed using Imaris software as following. An area the size of $1 \mathrm{~mm}^{2}$ in cortical white matter as well as striatum was chosen and confocal z-stacks (step size: $0.8 \mu \mathrm{m}$ ) were acquired with a Leica TCS SP5 confocal microscope (40x objective). A three dimensional image was generated In Imaris' Surpass view. Then the "surface" option in tool bar was selected, in the third channel (Far red), a region of interest including lipofuscin compartment within a single cell was segmented. The threshold was manually set in a way to carefully cover the whole volume of compartment in the cell by creating a surface. By pressing the end bottom in the software the volume of lipofuscin was automatically calculated, and could be exported as an excel sheet. Normally, 50 single cells were analyzed.

\subsubsection{Microglia-myelin contact}

Confocal z-stacks (step size: $0.8 \mu \mathrm{m}$ ) were taken in striatum with $40 \mathrm{X}$ objective of a Leica TCS SP5 confocal microscope. Then a three dimensional image from the whole area was created using surpass view in Imaris software. To measure the surface area of each microglia cell in contact with myelin, first, a single cell, including cell body and all the processes in total focus, was segmentad then by choosing "spots" option in tool bar and adjusting the appropriate threshold, the whole cell was covered. Second, the whole area labeled with MBP against myelin around the cell of interest was 
segmented; by activating the "Surface" item in tool bar a surface was created over that area. Total number of spots (representing cell process or cell body) and also number of close spots to the surface (representing myelin) was calculated using distance threshold 0.4. Finally, using these numbers the percentage of cell area in contact with myelin was calculated.

\subsubsection{Isolation of lysosomal inclusions from microglia}

Nukina and Ihara in 1985 described a protocol, based on ultracentrifugation and detergent (Sarkosyl) insolubility, to isolate protein aggregations such as tau paired helical filaments form Alzheimer's disease brain as well as indigestible lysosomal inclusions such as lipofuscin accumulated in aged brain. Therefore, in this project, we used the same protocol to isolate the lysosomal inclusions from microglia cells in aged mice for biochemical analysis. To do that, first we isolated microglia from old mouse brain.

\subsubsection{Microglia isolation}

For optimal dissociation of tissue samples, brain tissue from 12-month-old C57BI/6 wild type mice was dissociated using a Neural Tissue Dissociation Kit (Papain) (Miltenyi Biotec). Briefly, the mouse was perfused by cold 1x PBS, the brain was taken out and cut into small pieces then the tissue got dissociated using enzyme mix 1 containing papain for 15 minutes at $37^{\circ} \mathrm{C}$, and enzyme mix 2 for 10 minutes at $37^{\circ} \mathrm{C}$. Afterwards, the tissue was dissociated mechanically by wide and narrow-tipped pipettes until no tissue pieces remained. The suspension was applied to a $40 \mu \mathrm{m}$ cell strainer, and washed twice with Hank's balanced salt solution (HBSS). To remove myelin, the tissue pellet was resuspended in 37\% Percoll (Sigma) and overlaid on 70\% Percoll in DMEM containing 2\% FCS (Fetal Calf Serum), centrifuged at 500g for 30 minutes with no break. A thick myelin fraction which was formed over $37 \%$ percoll gradient was totally removed using vacume pump. A Thin fraction, containing single cell suspension, in the interface between $37 \%$ and $70 \%$ percoll was then carefully taken out and washed with the medium and MACS rinsing solution (0.5\% BSA and 2mM EDTA in PBS 1X). Microglia were then isolated from that single-cell suspension by MACS ${ }^{\circledR}$ Technology. The suspension was incubated with CD11b (Microglia) MicroBeads (Miltenyi Biotec) at $4^{\circ} \mathrm{C}$ for 15 minutes, after washing with MACS rinsing solution, the pellet was resuspended in $500 \mu l$ of the same buffer, applied on a MACS column placed in the magnetic field, following three times wash with $500 \mu l$ MACS buffer, CD11b positive cells (microglia) were then flushed out of the column, centrifuged at $400 \times \mathrm{g}$ for 8 minutes at $4^{\circ} \mathrm{C}$. The pellet was resuspended in $1 \mathrm{ml} 1 \times$ PBS and washed one more time. The final pellet was flash frozen in liquid nitrogen, and stored at $-80^{\circ} \mathrm{C}$ for future use. 


\subsubsection{Sarkosyl-insoluble fractionation of microglia}

The final pellet containing $1.5 \times 10^{6}$ isolated microglia cells of 12 -month-old mice was rsuspended in PBS, and divided into two portions. One part, called cell lysate, was prepared to be used directly for western blotting later. The other part was used for Sarkosyl-insoluble fractionation, resuspended in $300 \mu \mathrm{l} 10 \%$ sarkosyl and $1 \mu \mathrm{l}$ of $10 \mu \mathrm{g} / \mathrm{ml} \beta$-mercaptoethanol and incubated at $4^{\circ} \mathrm{C}$ for 4 hours on a roller. Afterwards, to separate Sarkosyl-insoluble fraction of the cells, the solution was transferred in Beckman $1.5 \mathrm{ml}$ tubes, and centrifuged at $130000 \mathrm{xg}$ for $35 \mathrm{~min}$ at $4^{\circ} \mathrm{C}$. The pellet was resuspended in $1 \mathrm{ml}$ cold $1 \times$ TBS $(50 \mathrm{mM}$ Tris $\mathrm{pH} 7.6,150 \mathrm{mM} \mathrm{NaCl})$ and, centrifuged again at $130000 \times \mathrm{g}$ for $35 \mathrm{~min}$ at $4^{\circ} \mathrm{C}$. The resulting pellet was washed one more time in cold $1 \times$ TBS. the supernatants was removed carefully and the final pellet (Sarkosyl-insoluble fraction, SIF) was flash frozen and stored at $-80^{\circ} \mathrm{C}$ for further use.

To examine the solubility of myelin membrane in Sarkosyl, the same experiment was done with $1.5 \mu \mathrm{g}$ pure myelin membrane isolated from 2 months old wild type mice as following.

\subsubsection{Myelin isolation and purification}

The myelin from 8-week-old C57BL/6 mice brains was isolated by sequential centrifugation on discontinuous sucrose gradient according to a protocol previously described (Norton and Poduslo, 1973) with some modifications. The ultracentrifugation was done using SW41 Ti rotor. The brain tissues were homogenized with a Dounce homogenizer in a solution containing 10mM HEPES, 5mM EDTA, 0,3M sucrose, and protease inhibitor. The homogenized tissue was layered on a sucrose gradient composed of $0,32 \mathrm{M}$ and $0,85 \mathrm{M}$ sucrose prepared in 10mM HEPES, $5 \mathrm{mM}$ EDTA. (pH 7,4), centrifuged at $75000 \mathrm{~g}$ for 30 minutes with low deceleration and acceleration. The crude myelin fraction was removed from interface, resuspended in distilled water, and centrifuged at 75000g for 15 minutes. The pellet was subjected to two rounds of hypo-osmotic shock by resuspension in $10 \mathrm{ml}$ ice-cold water, centrifuged at $12000 \mathrm{~g}$ for 10 minutes. For purification of myelin, the pellet obtained from the last step was dissolved in HEPE/EDTA buffer, and placed over the sucrose gradient; all the centrifugation steps and hypo-osmotic shocks were repeated as before. Eventually, the purified myelin pellet was resuspended in $1 \mathrm{ml} \mathrm{HEPE/EDTA}$ buffer and stored at $-20^{\circ} \mathrm{C}$. 


\subsubsection{Western blotting}

\section{SDS-PAGE}

To detect proteins in isolated sarkosyl-insoluble fraction (lipofuscin), sodium dodecyl sulfate polyacrylamide gel electrophoresis (SDS-PAGE) was performed as described previously. (Burnette,1981; Laemmli, 1970Natalia thesis). 12\% two-layer gel was prepared with Bio-Rad MiniPROTEAN 3 casting system as following:

Stacking gel $(10 \mathrm{ml})$

\begin{tabular}{ll}
\hline Component & Amount \\
\hline $30 \%$ Acrylamide/bis-acrylamide & $1.3 \mathrm{ml}$ \\
$0.5 \mathrm{M}$ Tris-HCl pH 6.8 & $2.5 \mathrm{ml}$ \\
$10 \%$ SDS & $0.1 \mathrm{ml}$ \\
$10 \%$ ammonium persulfate (APS) & $0.05 \mathrm{ml}$ \\
N'N'N'-tetramethylethylene diamine (TEMED) & $0.005 \mathrm{ml}$ \\
\hline
\end{tabular}

Resolving gel (12\%) (10ml)

\begin{tabular}{ll}
\hline Component & Amount \\
\hline $30 \%$ Acrylamide/bis-acrylamide & $4 \mathrm{ml}$ \\
$1.5 \mathrm{M}$ Tris- $\mathrm{HCl} \mathrm{pH} 8.8$ & $2.5 \mathrm{ml}$ \\
$10 \%$ SDS & $0.1 \mathrm{ml}$ \\
$10 \%$ ammonium persulfate (APS) & $0.05 \mathrm{ml}$ \\
N'N'N'-tetramethylethylene diamine (TEMED) & $0.005 \mathrm{ml}$ \\
\hline
\end{tabular}

The resolving gel was poured to $2 / 3$ height of casting system, the remaining part of system was filled with isopropanol to ensure a horizontal interface. After polymerization, isopropanol was removed, the stacking gel was added and a comb was adjusted.

The equal volume of all protein samples were dissolved in protein sample buffer (see below) and boiled at $90^{\circ} \mathrm{C}$ for $5 \mathrm{~min}$ to denature proteins. 
Protein sample buffer 6X:

\begin{tabular}{ll}
\hline SDS & $1.2 \mathrm{~g}$ \\
Bromophenol blue & $6 \mathrm{mg}$ \\
Glycerol & $4.7 \mathrm{ml}$ \\
Tris 0.5M pH 6.8 & $1.2 \mathrm{ml}$ \\
DTT & $0.93 \mathrm{~g}$ \\
$\mathrm{ddH}_{2} \mathrm{O}$ & $2.1 \mathrm{ml}$ \\
\hline
\end{tabular}

The gel cassette was adjusted into a clamping frame, placed in the tank filled with PAGE buffer (see below). $15 \mu$ l of sample was loaded onto the gel and the electrophoresis was performed with 100 voltages.

\section{PAGE buffer}

$0.25 \mathrm{M}$ Tris base

1.92 M Glycine

$1 \%$ SDS

\section{Western Blot}

After electrophoresis, western blot was performed to detect MBP in the cell lysate, Sarkosylinsolubel fraction of microglia cells and pure myelin membrane using a polyclonal MBP antibody. After separation with SDS-PAGE, the proteins were transferred from the gel onto the nitrocellulose membrane using the mini Trans-Blot Module. The gel sandwich in blotting cassette was set up and placed in the tank of the module containing transfer buffer ( $0.25 \mathrm{M}$ Tris base, $1.92 \mathrm{M}$ glycine, $20 \%$ methanol) and applied with $100 \mathrm{~V}$ for $1 \mathrm{hr}$.

Afterwards, the membrane was washed in PBST (0.1\% Tween 20 in PBS) for 10 min, immersed in $4 \%$ skim milk powder in PBST as a blocking solution for $30 \mathrm{~min}$ at RT and then incubated with primary antibody in PBST at $4^{\circ} \mathrm{C}$ overnight. After washing the membrane 3 times, each $10 \mathrm{~min}$, with PBST, it was incubated with horse radish peroxidase (HRP)-conjugated secondary antibody in PBST for one hour at RT. Then targeted protein was detected with enhanced chemiluminescence method using equal volumes of Luminol enhancer and peroxide solutions (Pierce/Thermo Scientific) covering the membrane at dark The light emission was detected with X-ray films, which were scanned with a conventional scanner. 


\subsubsection{In vivo endocytosis assay}

Mice were anesthetized intraperitoneally with a solution $(0.15 \mathrm{ml} / 25 \mathrm{~g})$ containing $4 \%$ Rompun $^{\mathrm{TM}} 2 \%$ (xylazine) (Bayer DVM for veterinary professionals) and $12.5 \%$ ketamine $10 \%$ (Medistar) in $0.9 \% \mathrm{NaCl}$, placed into stereotaxic apparatus (Kopf Instruments), and 1.5 $\mu$ g FITC-conjugated Dextran (40kDa; Molecular probes, Eugene, OR, USA) in sterile PBS $1 \mathrm{x}$ was injected by a glass capillary microinjector at the following coordinates relative to bregma: $0.3 \mathrm{~mm}$ anterior, $1.2 \mathrm{~mm}$ lateral and $1.2 \mathrm{~mm}$ below cortical surface. 7 hours after injection the mice were perfused and the brain tissues were prepared and stained as described before.

\subsubsection{Cuprizone treatment}

Six weeks after tamoxifen injection, Rab7 conditional knockout mice as well as corresponding control mice were treated with $0.2 \%$ cuprizone for four weeks to complete demyelination course in the central nervous system, following that the animals received normal diet for another four weeks to induce remyelination. Some groups were fed with the normal food 5, 11, 19, and 33 weeks after completed remyelination (recovery). Age-matched controls received normal diet without cuprizone throughout the whole experiment.

\subsubsection{Ethics Statement}

All experiments were approved and conducted in accordance with animal protection laws approved by the Government of Lower Saxony, Germany. The Approval ID is 33.7-42502-04-12/0778. C57BL/6 mice were used for all experiments. They were kept in groups of three in standard plastic cages and maintained in a temperature-controlled environment $\left(21 \pm 2^{\circ} \mathrm{C}\right)$ on a 12 -h light/dark cycle with food and water available ad libitum.

\subsubsection{Statistics}

Statistical analysis was done using GraphPad Prism (GraphPad Software, Inc.). To compare two groups, a Student's $t$-test was used. One-way ANOVA was performed for comparison of more than two groups. A p value of $<0.05$ was considered significant in all the tests. All values are represented as mean $\pm \mathrm{SD}$. 


\section{chapter 3}

\section{Results}

In this study we tested the possibility that microglia are involved in normal turnover of myelin during aging.

\subsection{Myelin breakdown during normal aging}

The brain tissues from 7 and 18 months old mice were prepared for electron microscopy as previously described. Myelin fragments were detected in extracellular space and also inside the cells in the white matter including corpus callosum and cortical white matter. Quantification of the number of extracellular myelin fragments in different age groups showed a significant increase with time.
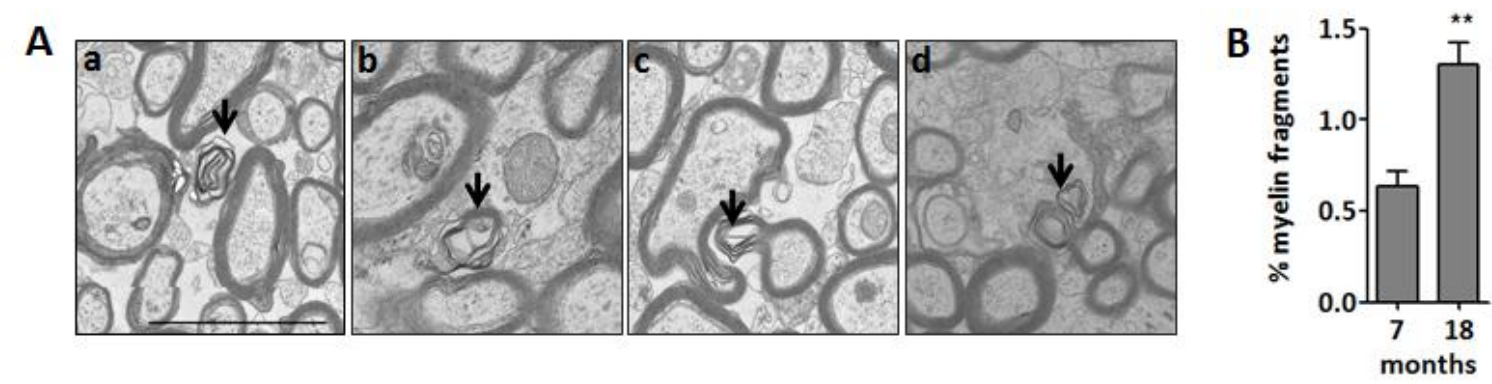

Figure3.1 Myelin fragments during normal aging in wild type mice. A) Electron microscopy images from an 18-month-old mouse showing myelin fragments in different positions: (a) in the extracellular space, (b) inside the cell, (c) a myelin fragment tearing away from the axon (d) a myelin fragment being engulfed by a cell, and. Scale bar: $2000 \mathrm{~nm}$. B) Quantification of myelin fragments number in 7 and 18 months old wild type mice. There is an increase with tim. Mean value+SD were indicated. ${ }^{* *} p<0,05$. Student's t-test.

\subsection{Microglia are involved in myelin turnover in normal aging}

\subsubsection{Microglia are in contact with myelin in wild type mice}

To visualize microglia and compact myelin in the parenchyma, we used Iba1 and MBP antibodies, repectively, the brain slices of wild type mice at 2, 4, 9, 18, and 24 months of age were used for histological studies to assess the contact of microglia with myelin. Confocal imaging of 18 and 24 months old mice showed that microglia form contact with myelin fragments via their processes. 
In addition, we quantified the size of microglia-myelin contact by measuring the cell surface area in contact with myelin using Imaris software as previously described. The size of contact was quantified mainly in striatum where the processes of microglia were more in focus due to less compaction of myelin compared to corpus callosum. The results showed that with age the size of cell area in contact with myelin significantly increased. (Fig. 3.2)

A

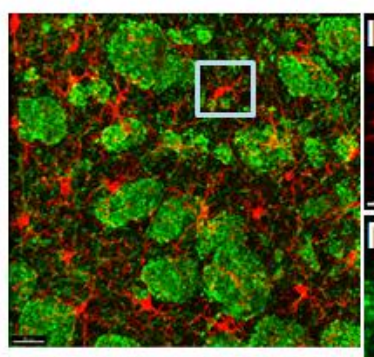

Iba1 MBP
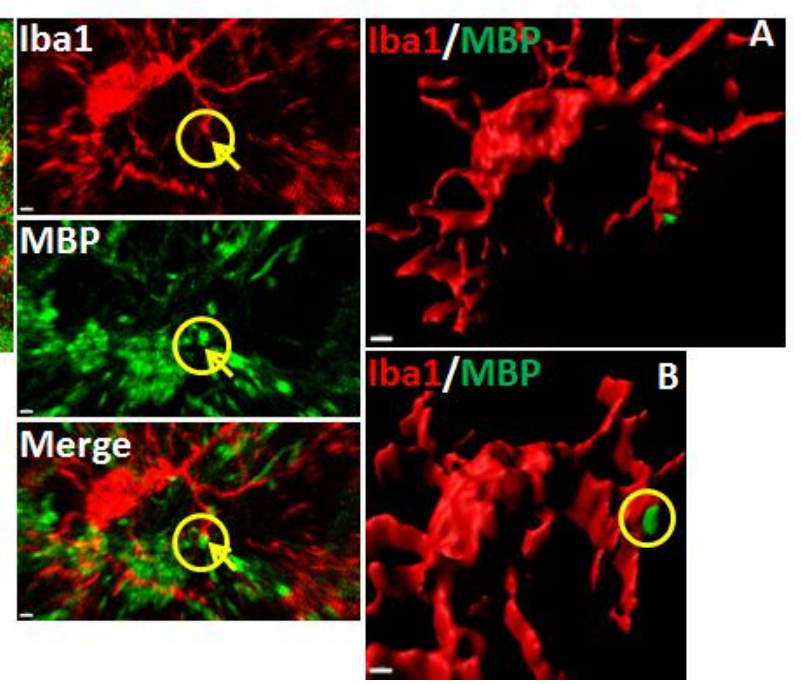

B

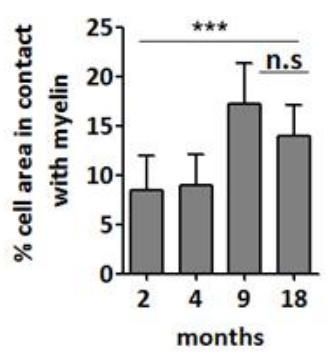

Figure3.2 Microglia are in contact with myelin in normal aging. Microglial and compact myelin in the striatum of $2,4,9,18$, and 24 months mice were labeled using Iba1 (red) and MBP (green) antibodies; confocal images were captured by acquiring Z-stacks. A) Confocal image from striatum in 18 months old wild type mice, Scale bar: $30 \mu \mathrm{m}$. a-c) the myelin fragments (Labeled with MBP in green) in myelinated areas are detected in close contact with the processes of microglia (Labeled with Iba1 in red). (d) and (e) 3D reconstruction of a microglia cell in contact with a myelin fragment. Scale bar: $2 \mu \mathrm{m}$. (C) Quantification of contact size of microglia with myelin is shown as mean + SEM $(n=4)$. The bigger contact size was detected in 9 months of age in wild type mice. Statistically significant differences are indicated by asterisks $(* * * p<0.0001)$. There is no significant difference (n.s) in the contact size between 9 and 18 months old mice $(p=0.6)$.

\subsubsection{Myelin fragments are engulfed by microglia}

Using immunohistochemistry myelin fragments labeled with MBP were detected at 18 and 24 months of age of wild type mice. These fragments which were mainly observed in cortical white matter and striatum but less in the corpus callosum were found within microglia at the age of 18 and 24 months. (Fig. 3.3) 
A

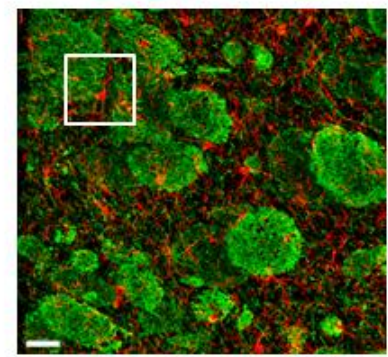

Iba1 MBP
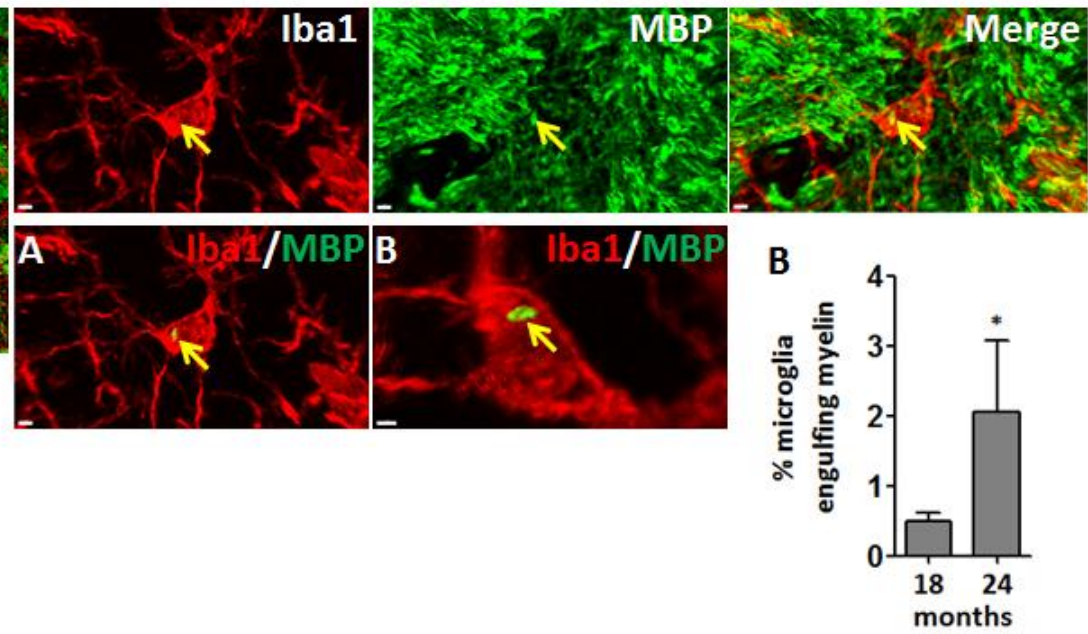

Figure 3.3 Engulfment of myelin fragments by microglia in normal aging. A) Confocal image of the striatum of a 24-month-old mouse. Scale bar: $30 \mu \mathrm{m}$. (a-e) A microglia cell with internalized myelin fragment. (d) 3D reconstruction showing the myelin fragment inside the cell. Scale bar: $2 \mu \mathrm{m}$. (e) 3D reconstruction of the same cell in a rotated position. Scale bar: $1 \mu \mathrm{m}$. C) Quantification of the number of cells with internalized myelin fragment. $n=3$, Mean+SD was reported, ${ }^{*} p<0.05$. Student's t-test.

\subsubsection{Density and lysosomal activity of microglia cells increase in the white matter}

Regarding the regional difference in microglia phenotype in the central nervous system in adult mouse (Hart, Wyttenbach et al. 2012), we focused on changes in microglia phenotype in white matter in normal aging. We considered the size of lysosomes as an indication of lysosomal activity in microglia. The brain tissues from 2, 4, 7, 9, and 24 months old wild type mice were co-labeled by Iba1 and LAMP1 antibodies; a series of z-stack confocal images within $30 \mu \mathrm{m}$ volume of brain slice was acquired and the volume of lysosomes were measured with Imaris software. Enlarged lysosomes were detected in microglia from 7 months of age whereas in 2 and 4 months old mice there was almost no LAMP1 positive microglia. (Fig. 3.4 A and B). Furthermore, variation in size of lysosomes in white matter versus gray matter was analyzed by measuring the volume of lysosomes within microglia in 7, 9, and 24 months old animals. In all groups there was a significant increase in the size of lysosomes in the white matter in comparison to gray matter (Fig. 3.4 C). These results are consistent with previous work that has shown an increase in the number of ED1 positive microglia in corpus callosum with age (Hua, Schindler et al. 2012).

We labeled microglia cells with Iba1 using DAB staining in wild type mice at the age of 2,9,18, and 24 months old and counted the cells exclusively in corpus callosum (size of area $=0.3 \mathrm{~mm}^{2}$ ) of 5 animals in each group. The number of Iba1 positive cells in corpus callosum was significantly higher in 
18 and 24-month-old mice compare to 2 and 9 months old (Fig 3.4.D) as shown previously (Mouton, Long et al. 2002; Hua, Schindler et al. 2012; Poliani, Wang et al. 2015)

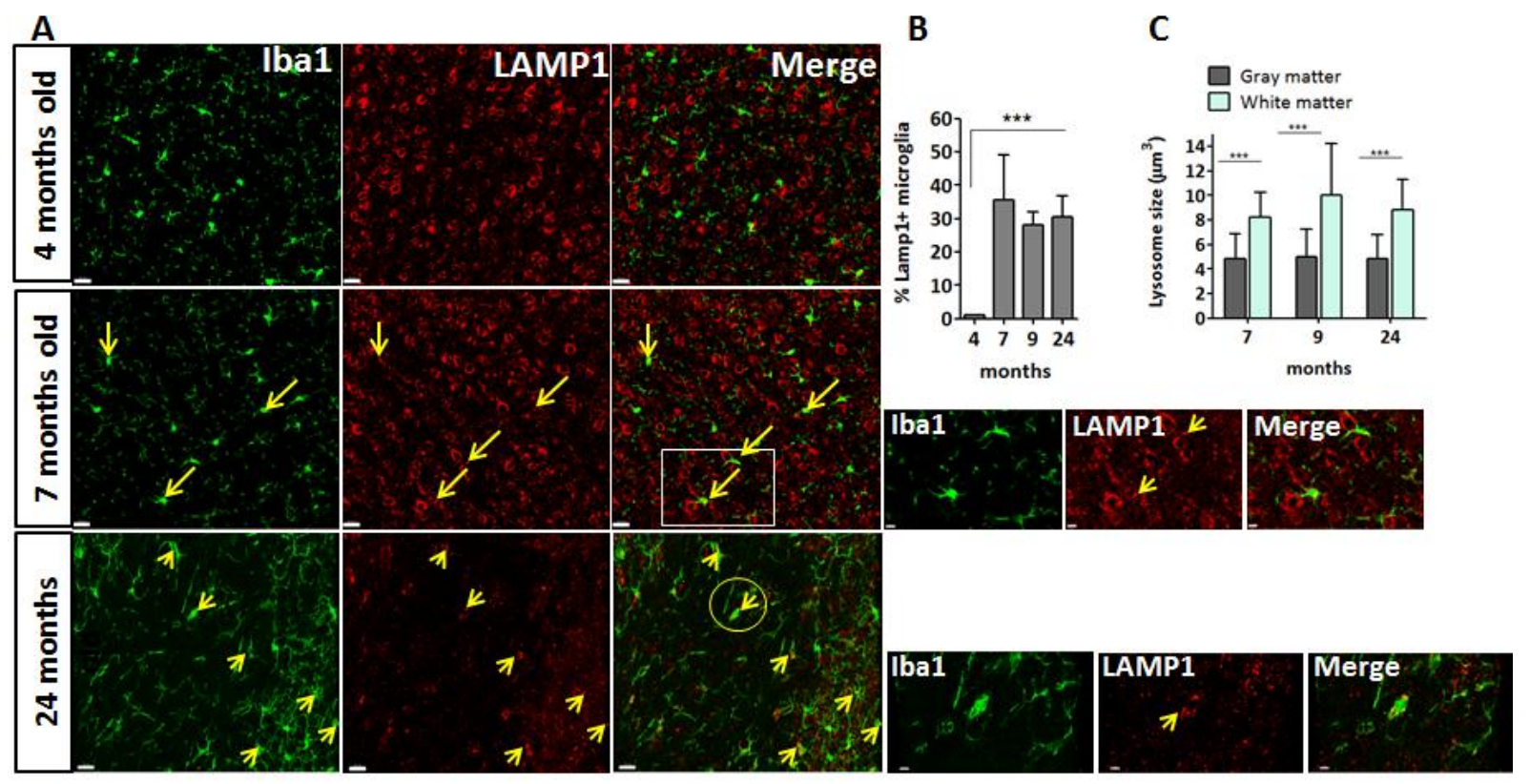

D

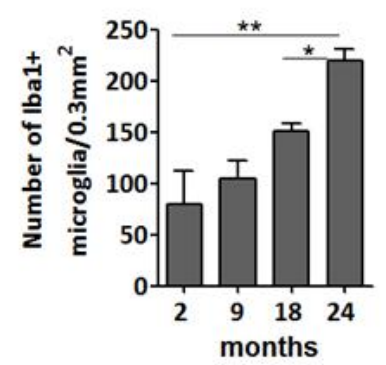

Figure3.4 Density and lysosomal size of microglia cells increase in white matter A) Visualization of LAMP1 (red) positive microglia (Iba1, green) in wild type mice. Lysosomes are not detectable within microglia in 4 months old mice. Lysosomes are observed in microglia at 7, 9, and 24 months of age. Scale bars: $20 \mu \mathrm{m}$ (overview) and $7 \mu \mathrm{m}$ (zoom in). B) Quantification of the number of lysosome positive cells at different age of wild type mice. C) Quantification of lysosome size in white matter versus gray matter. The size of lysosomes which were labeled by LAMP1 antibody was quantified by measuring the volume using Imaris software. In 7, 9, and 24 months old wild type mice the volume of lysosomes in white matter compared to gray matter was significantly larger. D) Quantification of cell number in corpus callosum of young, middle aged and aged wild type mice showed a significant increase in the number of cells with age. The statistics were done by t-test and one-way ANOVA. * $p$ $<0.05, * * p<0.01, * * * p<0.001$. Mean values plus SD were indicated. 


\subsubsection{Phagocytic activity of microglia in white matter of wild type mice}

We analyzed the expression level of galectin 3/Mac2, a galactose binding lectin, which is known to be upregulated in demyelination areas and involved in myelin phagocytosis (Smith 1999; Smith 2001). Mac2 antibody was used in DAB staining for labeling microglia in the stage of phagocytic activity on the brain tissues of $2,4,7,9,18$, and 24 months of age. Surprisingly, Mac2 positive microglia were detected only in aged (18 and 24 months old) mice but not in earlier time points. In addition, in these mice Mac2 positive populations were found mainly in myelinated areas including corpus callosum, anterior commissure, and medial septal nucleus (Fig. 3.5).

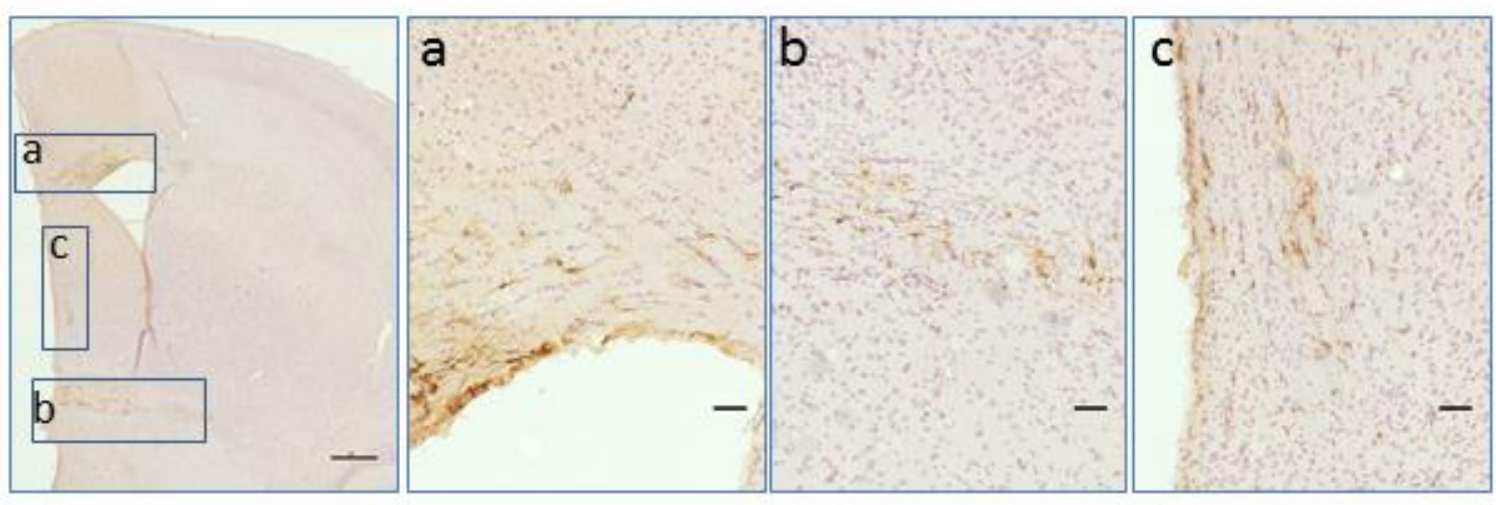

Figure 3.5 Detection of galectin 3/Mac2 positive microglial population during normal aging in wild type mice. Histological analysis of brain tissue of 24-month-old wild type mice using DAB staining showing a particular subtype of microglia which are galectin 3/ Mac2 positive and located in myelinated area including corpus callosum (a), anterior commissure (b) and medial septal nucleus

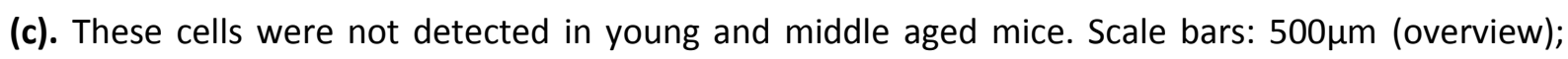
$50 \mu \mathrm{m}$ (zoom in).

\subsection{Cellular phenotypic alterations of microglia with age}

An increasing number of studies have shown that with age the functionality of microglia is impaired. Some changes in morphology and phenotype of microglia are listed in table 3.1 (Luo, Ding et al. 2010; Damani, Zhao et al. 2011; Hart, Wyttenbach et al. 2012; Harry 2013; Wong 2013). In this project we studied microglial characteristics in young ( 2 months old) and aged (18 or 24 months old) wild type mice to test whether myelin uptake by microglia during normal aging drives age-related alterations in the cells. 
Table 3. 1 characteristics of aged microglia

Intracellular accumulation of lipofuscin

retracted processes with less branches

Increased activation state

Decreased clearance function

Decrease in motility of processes

\subsection{1 lipofuscin granules accumulate within aged microglia}

Intracellular lipofuscin accumulation is a hallmark of microglia aging in the retina as well as brain, and studies on retina have shown that the amount of lipofuscin in RPE cells increases with age (Sierra, Gottfried-Blackmore et al. 2007; Tremblay, Zettel et al. 2012; Wong 2013). In our project we visualized and quantified the amount of lipofuscin within microglia in wild type mice. Images from 30 micron brain slices, labeled with lba1 using immunohistochemistry, were captured. To visualize lipofuscin we took advantage of autofluorescent feature of lipofuscin. Regarding the broad range of excitation and emission spectra for lipofuscin (excitation: 436-650,emission 450-700nm)(Barden 1980; Dowson 1982; Dowson, Armstrong et al. 1982; Sparrow and Duncker 2014), we found that when the brain tissue was excited at $633 \mathrm{~nm}$ the strongest autofluorescent signals from internalized lipofuscin was generated so that we could capture the images in far red channel (emission wavelength: 650-750nm) and used them for all the analysis. We measured the volume of lipofuscin within microglia using Imaris software The quantification showed a significant increase in the quantity of lipofuscin with time.

A

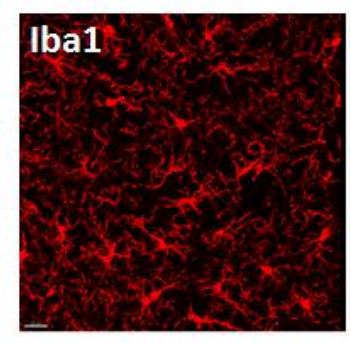

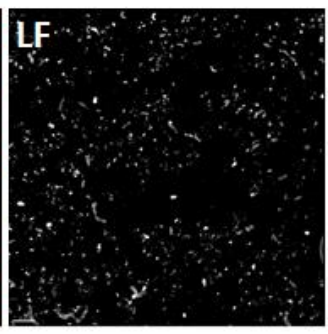

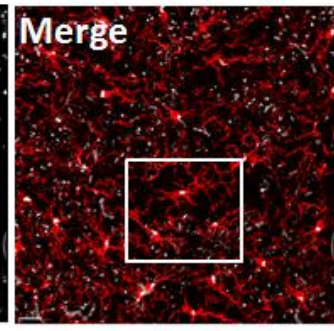

B

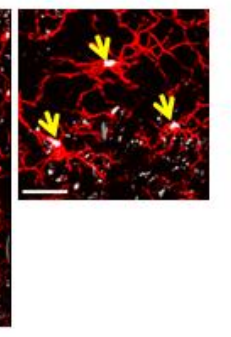

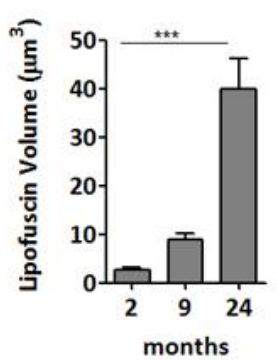

Figure3.6 visualization and quantification of lipofuscin granules in wild type mouse brain. A) Confocal images from wild type brain tissue shows intracellular lipofuscin (gray) in microglia (red) in 18 months old wild type mouse. Autofluorescent signal from lipofuscin was captured by excitation at $633 \mathrm{~nm}$ in far red channel. Scale bar: $20 \mu \mathrm{m} \mathrm{B)} \mathrm{Quantification} \mathrm{of} \mathrm{lipofuscin} \mathrm{volume} \mathrm{in} \mathrm{2,} \mathrm{9,} \mathrm{and} 24$ months old mice indicates an age-related increase in the amount of lipofuscin. ${ }^{* * *} \mathrm{P}<0.001$, one-way ANOVA. 


\subsubsection{The amount of lipofuscin in white and gray matter in aged wild type mice}

To test whether intracellular lipofuscin might contain remnants of internalized myelin membrane, we compared the amount of lipofuscin in white matter versus gray matter in 9, 18 and 24 months old wild type mice. Confocal z-stack images in withe matter including striatum, corpus callosum and cortical white matter and in cortical gray matter including layer I and II were captured. Then the volume of intracellular lipofuscin in 40 single microglia in both regions was quantified using Imaris software. Three mice were evaluated per age group. In the mice of each group, the size of lipofuscin in white matter was larger as compared to gray matter.

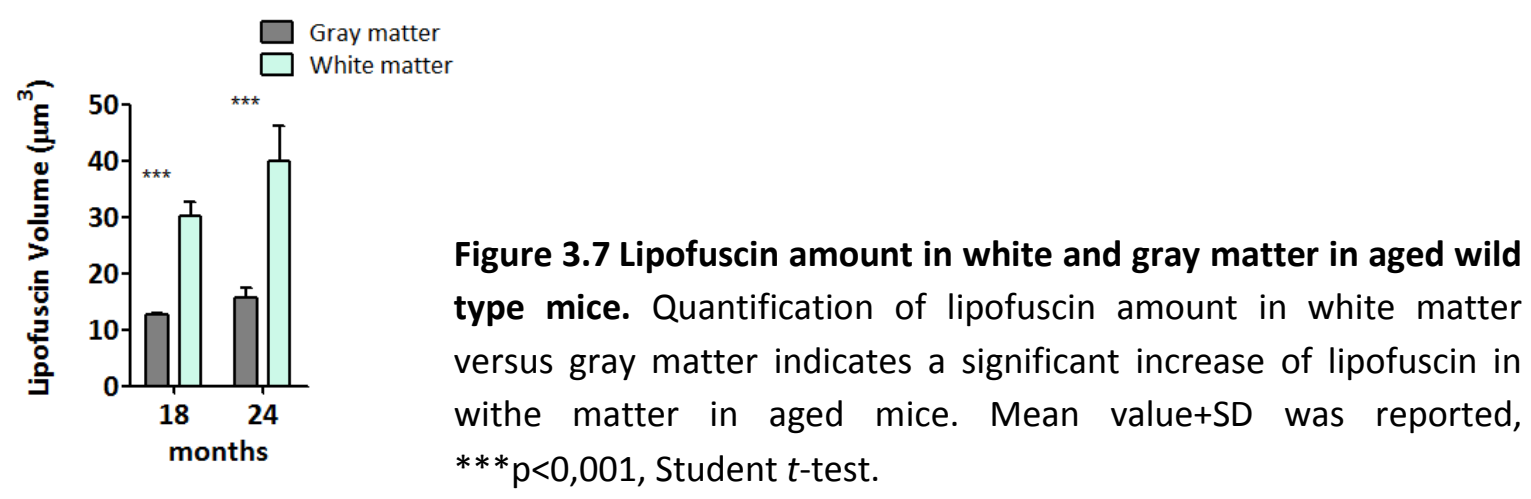

\subsubsection{Detection of myelin fragments within lipofuscin}

Given that myelin is engulfed by microglia during normal aging, and that lipofuscin accumulates within microglia with age, we hypothesized that lipofuscin might contain remnants of myelin with time. To test this, brain tissues from 18 months old mice were labeled with Iba1 and MBP. To visualize myelin overlapping with lipofuscin in separated channels we needed to remove autofluorescent signal produced by lipofuscin in green channel where MBP was captured. Therefore, we did dye separation using Leica software as previously described so that lipofuscin signal was exclusively captured in the far red channel. However, due to broad range of emission spectrum of lipofuscin we were not able to eliminate autofluorescent signal of lipofuscin from the red channel (Em: 570-630nm). Therefore, we used this channel for detection of Iba1 signal. Using this strategy we could detect myelin fragments (in green Channel) associated with lipofuscin (in far red channel) within microglia. The volume which was labeled with MBP overlapped with the volume occupied by lipofuscin in the cell. (Fig 3.8 A) We also labeled myelin with green Fluoromyelin (FM) and applied the same strategy as above. 3D reconstruction showed that internalized myelin fragments labeled with FM is partially included in intracellular lipofuscin (Fig 3.8 B). We also quantified the number of cells 
with internalized myelin within lipofuscin in 18 and 24 months old mice, and found out that the association between myelin and lipofuscin increases with time (Fig 3.8 C).

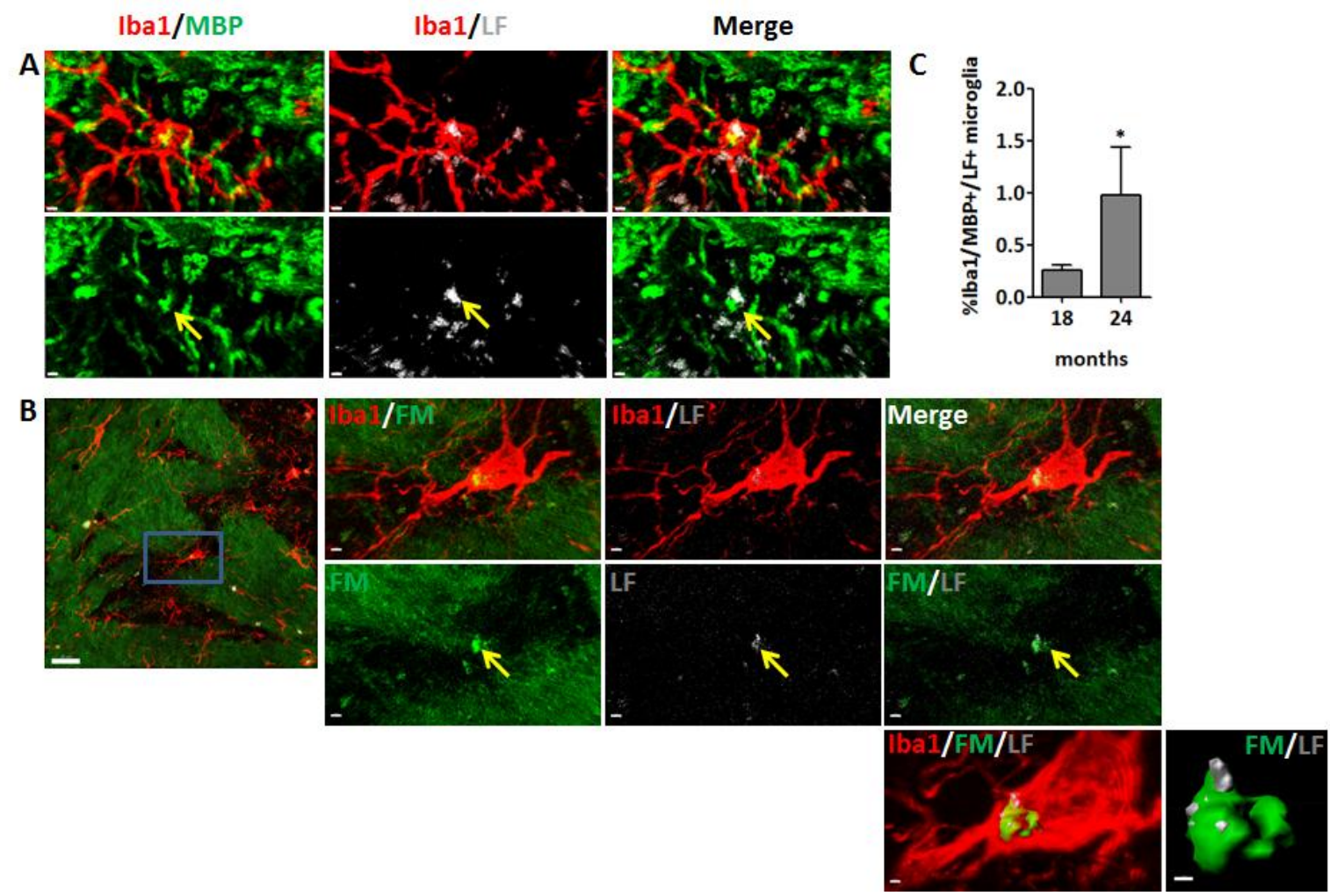

Figure 3.8 myelin fragments in the cells are associated with intracellular lipofuscin. A) co-labeling of myelin and lipofuscin using MBP antibody (green) and seperated autofluorescent signal (Far red) in 18 months old shows overlapping between internalized myelin fragment and intracellular lipofuscin. Scale bar: $2 \mu \mathrm{m}$. B) 3D reconstruction of myelin fragments labeled with fluoromyelin and intracellular lipofuscin in 24 months old mouse confirms the association of myelin and lipofuscin. Scale bar: $2 \mu \mathrm{m}$. C) Quantification of the number of microglia cells with internalized myelin included within lipofuscin representing an increase with time. ${ }^{*} p<0,05$. Mean value+SD.

\subsubsection{Biochemical characterization of lipofuscin contents}

To analyze myelin membrane remnants in lipofuscin we isolated and analyzed their content biochemically by Western blotting. For this, we isolated microglia from 12 months old mice (pooled from 8 mice) using neural tissue dissociation kit and MACS technology. The obtained pellet of the pure microglia cells at this stage was divided into two parts. One part used directly for Western blotting to define the amount of intracellular myelin. The other part was incubated in $300 \mu 10 \%$ Sarkosyl for four hours at $4^{\circ} \mathrm{C}$ to separate Sarkosyl-insoluble fraction (SIF). After ultracentrifugation, the final pellet was used for Western blotting to detect modified aggregated MBP in sarkosylinsoluble fraction of cell lysates. We isolated purified myelin membrane from wild type mice, and extracted it with Sarkosyl to isolate Sarkosyl-insoluble pellet and use for western blotting. 
An aggregation of a high-molecular weight $(70 \mathrm{kDa})$ fraction of MBP was found in cell lysate. After extraction in Sarkosyl the same fraction of MBP was recovered indicating that internalized MBP forms detergent-insoluble aggregates in microglia. In sarkosyl-insolube fraction of pure myelin membrane no MBP was detected indicating that MBP is soluble in sarkosyl.(Fig 3.9).

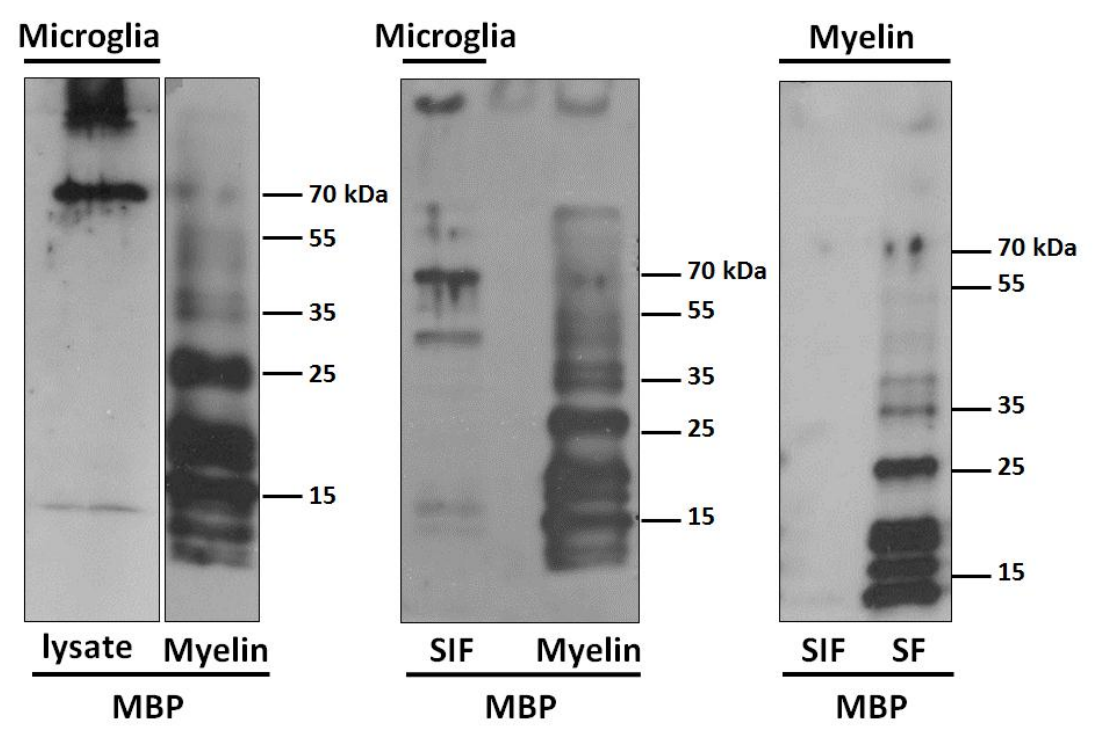

Figure3.9 Characterization of sarkosyl-insoluble fraction of isolated microglia. Left, Western blot analysis of purified microglia lysates from 12 months old mice shows MBP in the high-molecular weight region; MBP in myelin is shown as a reference in the right lane. Middle, high-molecular weight species of MBP existed in the Sarkosyl-insoluble (SIF) microglia membrane fraction (12 months old mice, 1 out of 5 representative experiments); MBP in myelin is shown as a reference in the right lane. Right, Sarkosyl extraction on purified myelin shows that myelin- associated MBP is Sarkosyl-soluble (Sarkosylsoluble membrane fraction, SF).

\subsubsection{Age-related changes in microglia shape}

Iba1 antibody was used to visualize microglia cells in the mouse brain by immunohistochemistry in $30 \mu \mathrm{m}$ thick sections. To obtain the whole volume of an individual cell, 40 image stacks were acquired in $z$ direction using confocal microscopy. Images were captured in different regions including corpus callosum, cortical white matter and gray matter. To assess the complexity and length of dendritic arbors of microglia the total area occupied by a single cell was measured by Imaris software as described before. The quantification was done in different region of brain for 40 cells per each mouse. The results showed that microglia in aged wild type mice (15 months old) have significantly smaller cell area than young mice ( 2 months old). Furthermore, all the cells with less branched processes contain large amount of lipofuscin (Fig 3.10). 

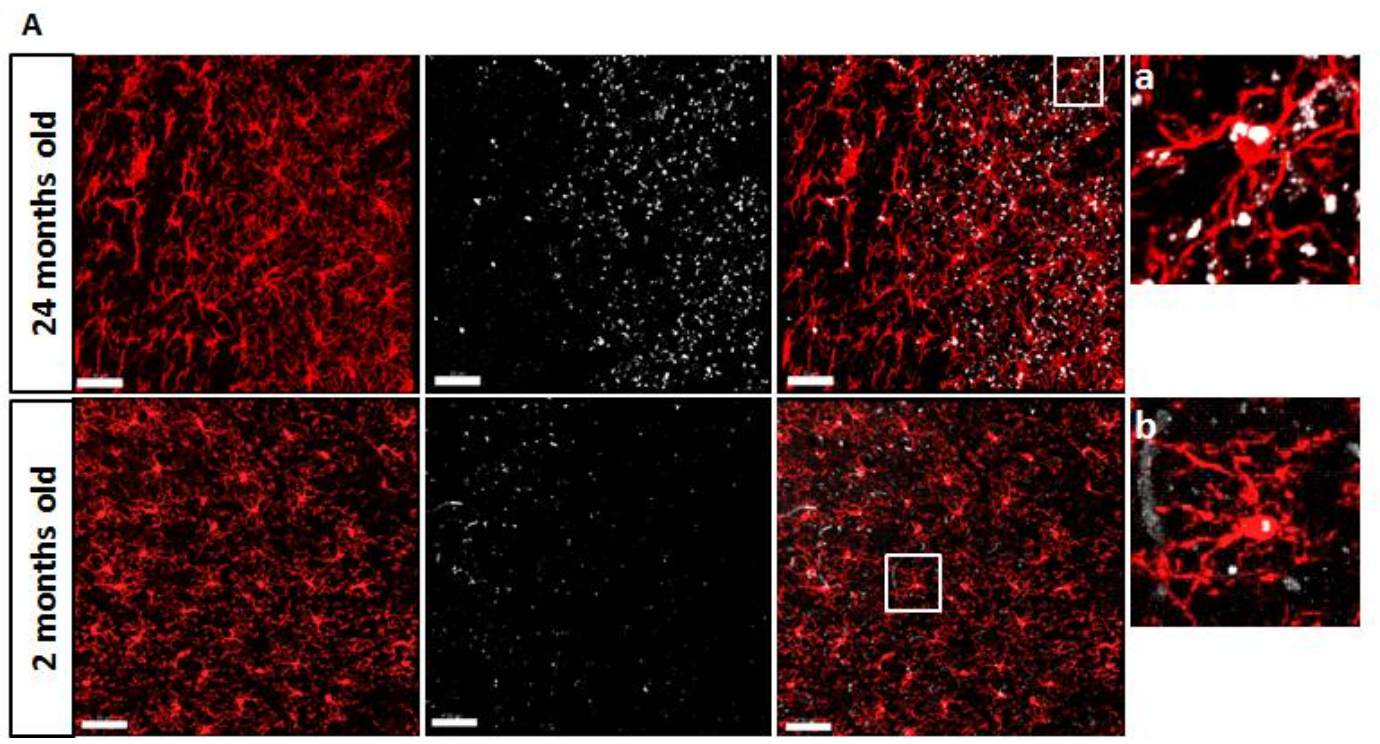

\section{B}

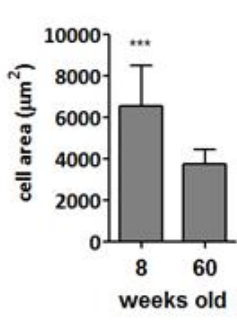

Figure 3.10 Aged microglia have less branched and shorter processes. A) The confocal images are showing a comparison between dendritic arborization of microglia in aged ( 24 months old) and young ( 2 months old) wild type mice. Whereas cell processes are much more branched and longer in 2 months old mice, they are less complex and more retracted in aged microglia. Scale bar: $50 \mu \mathrm{m}$. The images show that large lipofuscin granules are detected exclusively in the cells with less branched processes (a and b). B) cell area quantification showed a decrease in the size of cell area of microglia in 15 months old compared to 2 months old mice $(n=5)$. Mean values+SEM were indicated; Student's t-test. ${ }^{* * *} \mathrm{P}<0.001$.

\subsubsection{Less motility of microglia processes in aging brain related to lipofuscin accumulation}

It has been shown that microglia processes are less motile in aged brain, (Damani, Zhao et al. 2011; Wong 2013) and this may be related to shorter and less branched processes of these cells. We tested whether there is a correlation between accumulation of lipofuscin within microglia and the motility of their branches. To examine this, live imaging of green fluorescent-labeled microglia in CX3CR1 GFP/+ young ( 3 months old) and aged ( 24 months old) mice was done to compare process motility of lipofuscin positive microglia versus cells with no lipofuscin in young mice. In two dimentinal maximum-intensity projections of time-lapse recordings for 15 minutes the area occupied by a process was measured every recording period of 32. Variance between the size of process area calculated every 32 seconds in 2 months old mice was 70028,72 while the variance for 24 months old mice was 26839,47 . These numbers indicated that the branches of young microglia cells with almost no lipofuscin were more dynamic compared to microglia in old mice which accumulates lipofuscin (blue arrow (Fig 3.11). The calculation was done for 2 processes in 5 cells from each mouse. 

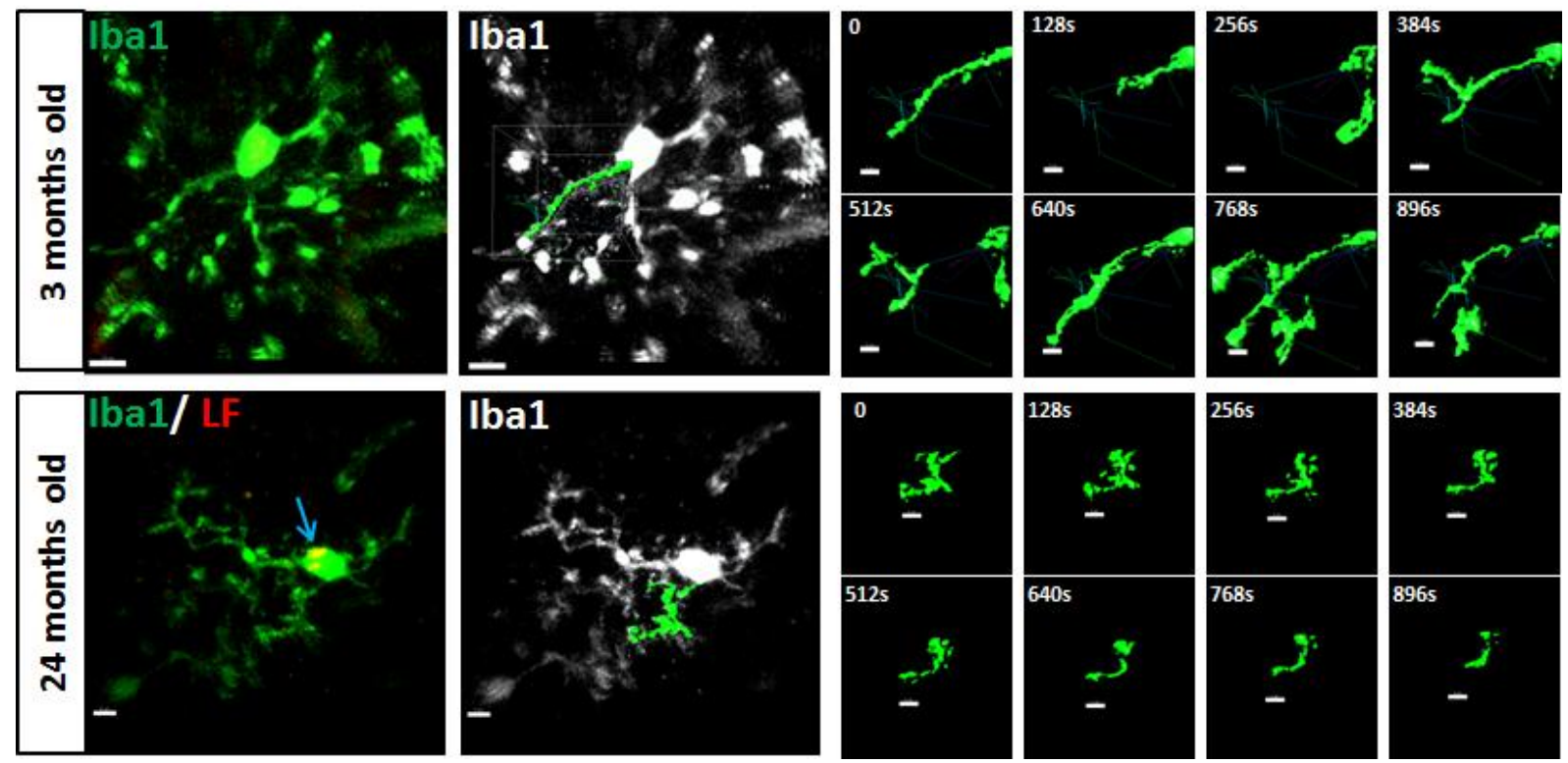

Figure 3.11 Dynamics of the motility of microglia processes in old and young mice regarding lipofuscin accumulation. Two dimensional maximum-intensity projections of time-lapse recordings from Green microglia in CX3CR1 ${ }^{\mathrm{GFP} /+}$ mice for 15 minutes were achieved. 3D Reconstitution of a process of microglia cell showing the variation in area occupied by the process in 3 month and 24 months old mice. The variation was considered every 128 seconds during recording period of 15 minutes. The process of microglia in young mouse shows a higher movement dynamics compared to that in old microglia containing a large amount of lipofuscin (blue arrow). Scale bar, 10 $\mu$ m (overview, 3 months old) and $5 \mu \mathrm{m}$ (overview, 24 months old, and zoom in).

\subsubsection{Age-related changes in activation state of microglia}

The changes in the basal activation state of aged microglia is shown in previous studies (Rogers, Luber-Narod et al. 1988; Sheng, Mrak et al. 1998; Frank, Barrientos et al. 2006; Miller and Streit 2007; Wong 2013). Our histological studies showed MHCII positive cells were found in aged mice (18-24 month old) (Fig 3.12). In addition, we found an upregulation of Mac2 (Galectin 3) in aged mouse brain which was mainly restricted to the white matter. While there was no up-regulation of Mac3 during normal aging, the expression of Fcy receptor II/III (CD16/CD32) and complement receptor 3 (Mac1) was detectable during normal aging, and there was no significant difference in the level of their expression among different age groups. Previous ex vivo and in vitro studies of aged microglia showed an increased expression of inflammatory cytokines including IL-1 $\beta$, TNF- $\alpha$, and IL- 6 (Ye and Johnson 1999; Sierra, Gottfried-Blackmore et al. 2007; Njie, Boelen et al. 2012). However, by performing quantitative PCR for isolated microglia none of these cytokines was detected in aged mice. The reason for our negative finding could be that the expression of these cytokines in physiological condition is so low that cannot be detected in vivo. 


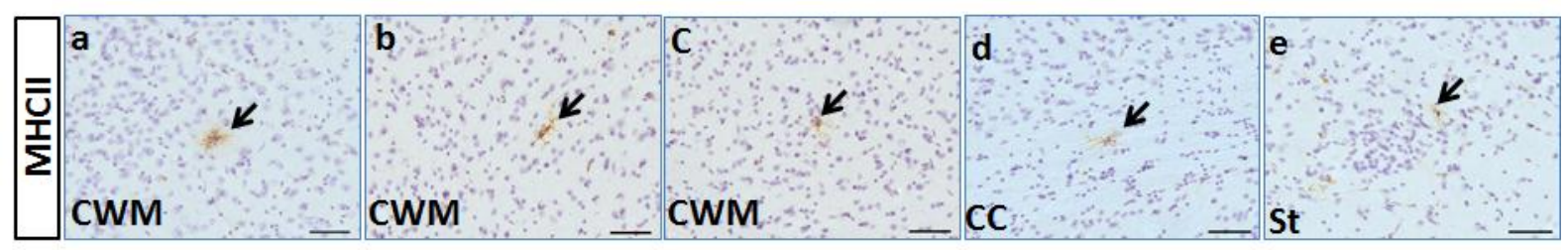

Figure 3.12 Analysis of the activation state of microglia in normal aging. DAB staining was done for brain sections of wild type mouse at the age of 2, 4, 7, 9, 18 and 24 months. Histological studies showed a small population of MHCll positive cells only in aged mouse brain (18-24 months old). The distribution of those cells is not restricted to a certain region of brain, they are found all over the brain. Scale bar: 50 $\mathrm{mm}$. CWM: Cortical White Matter, CC: Corpus Callosum, St: Striatum

\subsubsection{Clearance function of microglia in aging}

We hypothesized that due to age-related accumulation of lipofuscin in aged microglia, clearance function of cells may decline. Therefore, we injected FITC-conjugated Dextran into the brain of wild type mice at the age of 2 and 10 months old. There was a $20 \%$ reduction in the number of cells with internalized dextran in middle aged (10 months old) compared to young ( 2 months old) mice. (Fig 3.13).
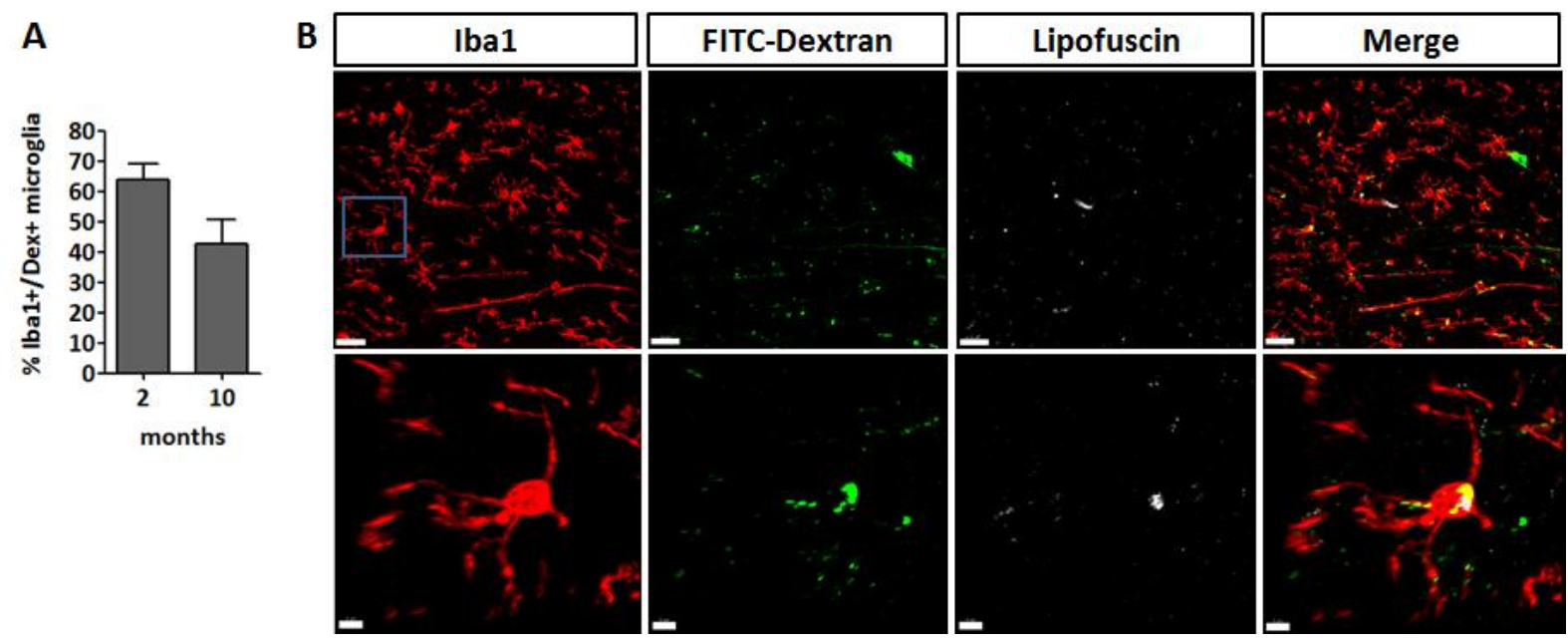

Figure 3.13 Clearance function of microglia in normal aging. A) Quantification of Dextran positive $($ Dex+) cell number in 2 and 10 months old wild type mice. $1.5 \mu \mathrm{g}$ FITC-conjugated Dextran was injected in the cortex of brain $(n=4)$ and 7 hours after injection, the mice were analyzed. Number of dextran positive cells in middle aged mice was $20 \%$ less than that in young animal. Mean value+SD was indicated. B) Confocal image of Dextran injection site in the cortex of brain in 10-month-old mouse showing that Iba1 (red)/lipofuscin (gray) positive cells engulfed Dextran (green). Scale bar: $30 \mu \mathrm{m}$ (overview) and $5 \mu \mathrm{m}$ (zoom in). 


\subsection{Enhanced myelin breakdown in demyelinating models and microglia behavior}

We speculated that increased myelin breakdown might induce aging-related changes in microglia. Therefore, we used three mouse models of demyelination, PLP overexpressing mice (a model for Pelizaeus-Merzbacher disease, (PMD mice), Cuprizone-induced demyelinating mice, and shiverer mice. In these mice, we analyzed three hallmarks of aging in microglia, lipofuscin formation, activation state, and uptake activity.

\subsubsection{Myelin in PMD mice and cuprizone model}

We analyzed PMD mice at the age of 2, 7, and 10 months old. These mice rarely survive beyond 10 months of age. Figure $3.14 \mathrm{~A}$ shows the rate of demyelination during this time. At the age of 2 months demyelination was not severe; compact myelin was detectable by MBP especially in corpus callosum. However, at later time points ( 7 and 10 months old) the level of demyelination increased showing myelin breakdown in the white matter.

In addition, we induced demyelination in wild type mice by Cuprizone treatment. In these mice demyelination was detectable mainly in corpus callosum 4 weeks after cuprizone treatment, and following 4 weeks of normal diet remyelination was induced. To distiguish between de and remyelination phase in cuprizone mice we used Fluoromyelin as shown in figure 3.14 B.
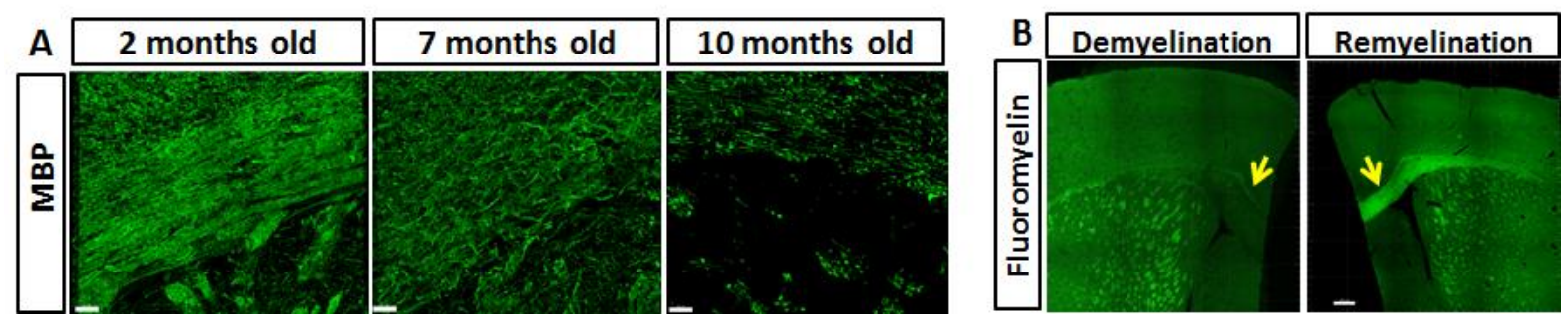

Figure 3. 14 An overview of demyelination rate in PMD and cuprizone mice. A) Myelin was labeled with MBP. 2-month-old PMD mice showed normal myelination while in 7 and 10 months old animals, compact myelin could not be completely detectable. Scale bar: $30 \mu \mathrm{m}$. B) de- and remyelination phases in cuprizone mouse. The compact myelin was labeled with Fluoromyelin. During demyelination the loss of myelin was detectable mainly in corpus callosum following 4 weeks of cuprizone diet. Completed remyelination was observed 4 weeks after feeding the mice with normal food. Scale bar: $300 \mu \mathrm{m}$. 


\subsubsection{Microglia in PMD mice and cuprizone model}

\subsubsection{Morphology and activation state}

\section{PMD mice}

In PMD mice microglia accumulation was observed in corpus callosum and striatum where myelin breakdown was severe. Myelin fragments were detected within microglia with very short and retracted processes (Fig $3.15 \mathrm{~A}$ ). In addition, DAB staining showed that all the cells were totally activated with high expression of Mac2 and MHCll. Compared to 2 month old mice, the population of activated cells in 7 and 10 months old was bigger (Fig 3.15 B).

A
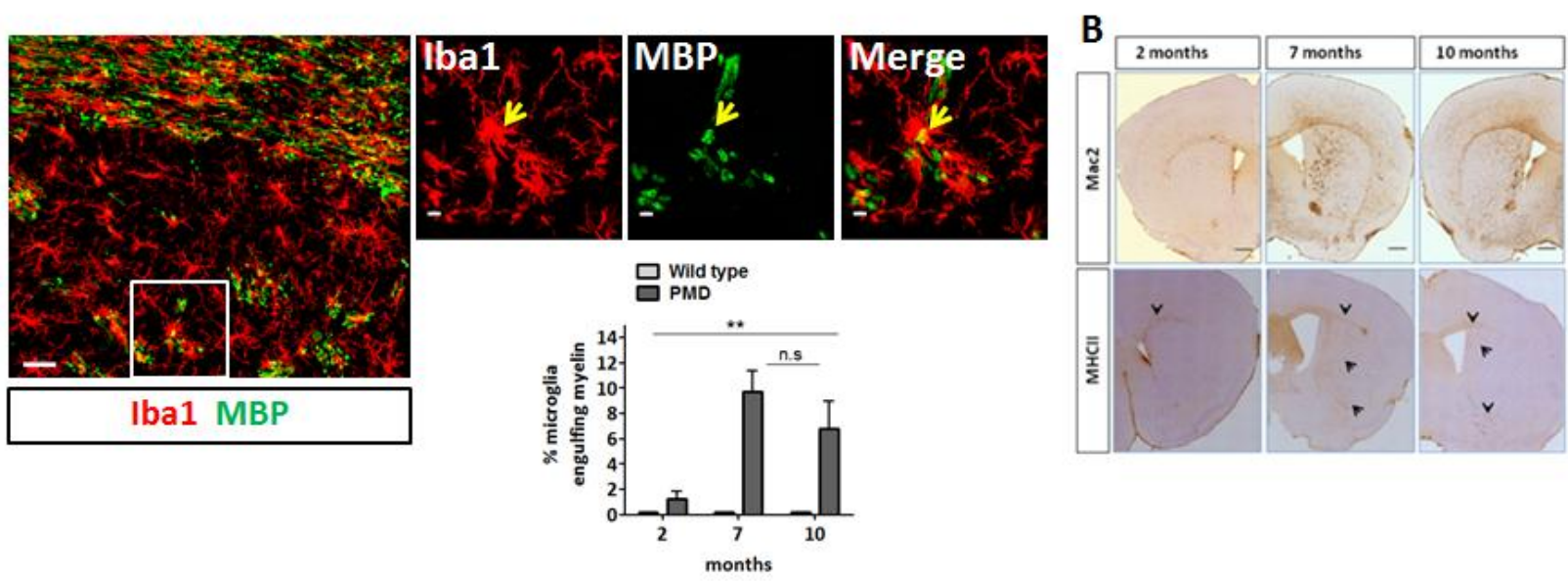

Figure 3.15 Morphology and activation state of microglia in PMD mice. A) Confocal images of brain tissues of PMD mice at the age of 10 months old showing myelin phagocytosis by microglia (Iba1, red) with highly retracted branches in areas with myelin breakdown (striatum). Scale bar: $30 \mu \mathrm{m}$ (overview) and $5 \mu \mathrm{m}$ (zoom in). Quantification of the number of microglia engulfing myelin fragments in PMD mice. Student' t-test, n.s: $\mathrm{P}=0.2865$, One-way ANOVA, ${ }^{* *} \mathrm{p}<0.01$, mean values $+\mathrm{SD}$ was reported. B) At 2 months of age, Mac2 and $\mathrm{MHCll}$ positive cells are detected mainly in corpus callosum while these cells are highly populated all over the white matter such as corpus callosum and striatum at 7 and 10 months of age. Scale bar: $500 \mu \mathrm{m}$.

\section{Cuprizone-treated mice}

In cuprizone-treated mice the expression of $\mathrm{Mac2}$ and $\mathrm{MHCll}$ in microglia was analyzed at different time points as shown in figure 3.16 A. 4 weeks after cuprizone treatment, when the demyelination was induced, a considerable number of $\mathrm{MHCll}$ positive cells were detected mainly in striatum and corpus callosum and a large population of Mac2 positive cells was found in the white matter as well. After 4 weeks of normal diet, when remyelination was ongoing, a considerable number of both Mac2 
and $\mathrm{MHCll}$ positive cells were still detectable. Activation state of microglia cells was analyzed at later time points after completed remyelination (see the timeline diagram in figure 3.16. A, T: Time point). 5 weeks after completed remyelination the expression of Mac2 and MHCII was mainly detectable in the corpus callosum. 11 and 19 weeks after completed remyelination, Mac2 was expressed by a small cell population in corpus callosum and striatum (Fig 3.16 B and D) whereas there was no MHCII positive cells detectable at these time points. (Fig 3.16 C and D) However, a significant number of $\mathrm{MHCll}$ reappeared 33 weeks (almost 8 months) after completed remyelination in the comissura anterior (Fig $3.16 \mathrm{C}$, i) Mac2 positive population was also found in the same region 33 weeks after complete remyelination (Fig $3.16 \mathrm{~B}, \mathrm{j}$ ).

A

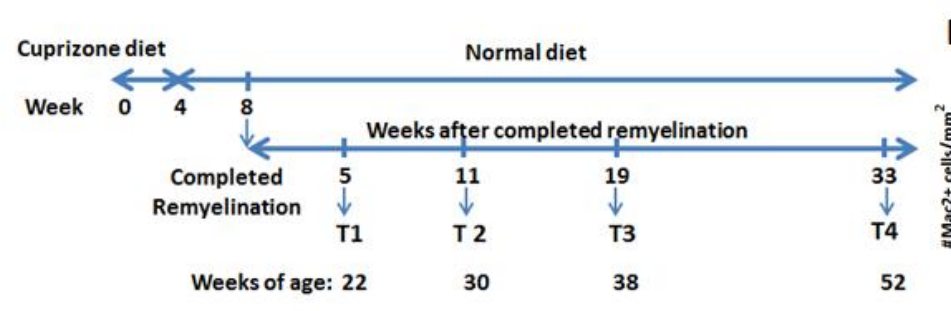

B

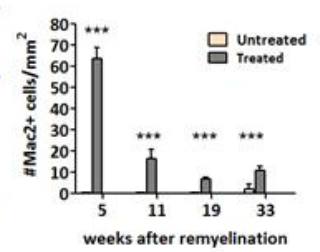

weeks after remyelination

19WPRe

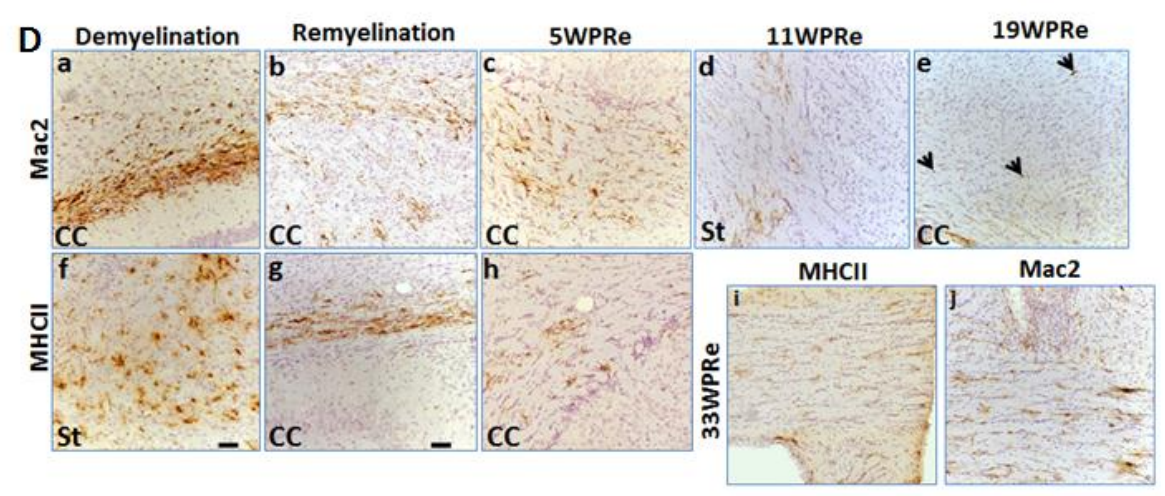

Figure 3.16 Activation state of microglia in Cuprizone mice. A) Timeline diagram for cuprizone mice analysis (T: Time point). B, C) Quantification of the expression of MHCII and Mac2 in cuprizone mice after completed remyelination compared to untreated age-matched group. $n=4$, Mean value+SD was indicated, $* * * p<0.001$. D) The expression of Mac2 (a-e) and MHCII (f-h) in cuprizone mice at different time points. 11 and 19 weeks after remyelination there was no $\mathrm{MHCII}$ positive cells detectable. (i and j) $\mathrm{MHCll}$ and Mac2 positive cells in anterior commissura 33 weeks after completed remyelination. CC: Corpus Callosum, St: Striatum. WPRe: Weeks Post Remyelinatio. Scale bar: $50 \mu \mathrm{m}$.

\subsubsection{Lipofuscin accumulation in microglia in demyelinating models}

Next we tested whether the amount of lipofuscin was affected in demyelinating models. Therefore, as described before, lipofuscin was visualized and quantified at different time points in both PMD and cuprizone-treated mice. Compared to age matched wild type mice, there was no significant 
change in the amount of lipofuscin in PMD mice at 2 months of age while it increased drastically at later time points (7 and 10 months old mice) (Fig $3.17 \mathrm{~A}$ and B). In cuprizone-treated mice, 5, 11, and 33 weeks after completed remyelination, lipofuscin increased significantly in comparison to age matched untreated mice (Fig $3.17 \mathrm{C}$ ).

Shiverer mice: In these mice, a population of unramified microglia with retracted unbranched processes was observed in the corpus callosum at 9 weeks of age. We detected lipofuscin inside those cells (Fig $3.17 \mathrm{E}$ ). In comparison with heterozygous shiverer mice, at 25 and 63 days of age there was a significant amount of lipofuscin in microglia in homozygous mice (Fig 3.17 D).
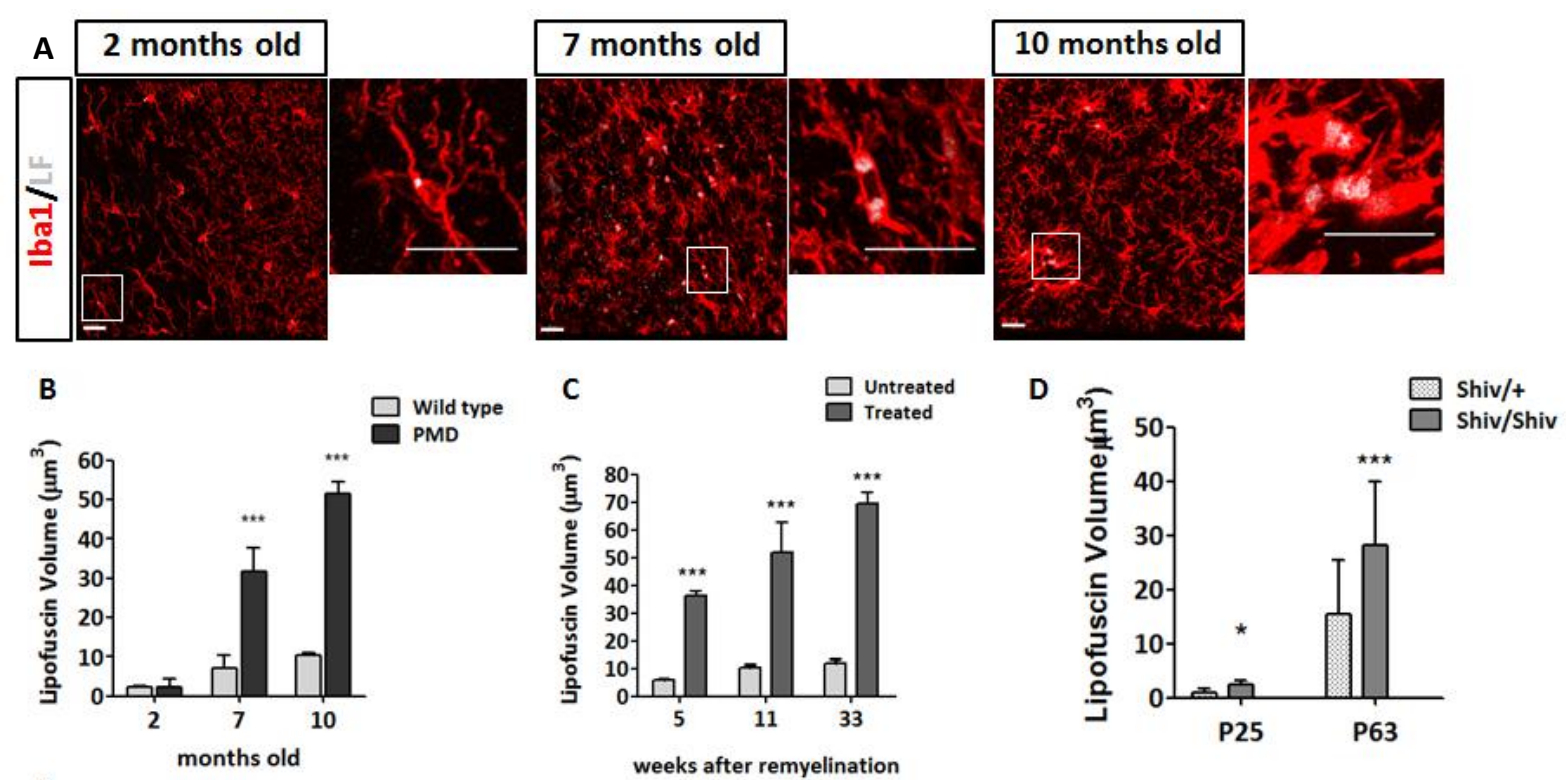

E
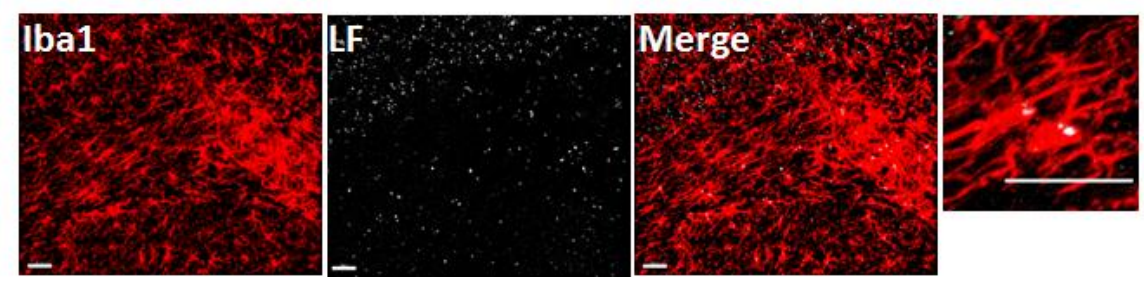

Figure 3.17 Lipofuscin formation and quantification in demyelinating models. A) Confocal images from PMD mice showing the development of intracellular lipofuscin (gray) in microglia (red), the size of lipofuscin increases with time. Scale bar: $20 \mu \mathrm{m}$. Quantification of lipofuscin amount in PMD (B) cuprizone-treated (C) and shiverer (D) mice at different time points. $n=3-4$ for each group of study. Mean $+S D$ was indicated, ${ }^{*} p<0.05, * * * p<0.0001$. E) Confocal images of microglia in corpus callosum in 9 weeks old shiverer mice. Microglia in this stage has retracted processes and intracellular lipofuscin, and they are aggregated in corpus callosum. Scale bar: $30 \mu \mathrm{m}$. 


\section{3 | Results}

\subsubsection{Detection of myelin fragments within intracellular lipofuscin in cuprizone mice}

Previously we found that lipofuscin partially contains myelin fragments in physiological condition when the mice were more than 18 months old (See section 3.3.1.2). To confirm that, we analyzed cuprizone mice at the latest time point (33 weeks after completed remyelination), when microglia have a large amount of lipofuscin compared to untreated mice and were in contact with myelin debris dring de- and remyelination. The mice were one year old at this time point. We labeled myelin with Fluoromyelin, microglia with Iba1, and visualized lipofuscin via autofluorescent signal. Using confocal images we could find co-localization of internalized myelin fragments with lipofuscin. In addition, the number of cells with Fluoromyelin-labeled myelin within lipofuscin was significantly higher in treated mice as compared to untreated mice (Fig $3.18 \mathrm{~B}$ )

A

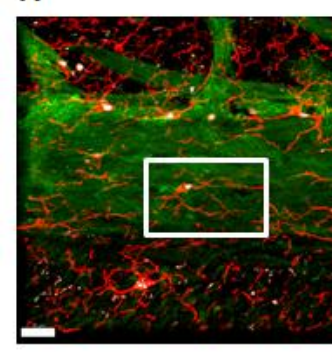

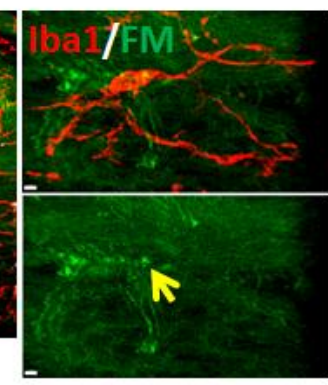

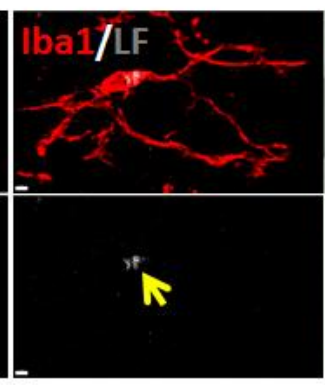

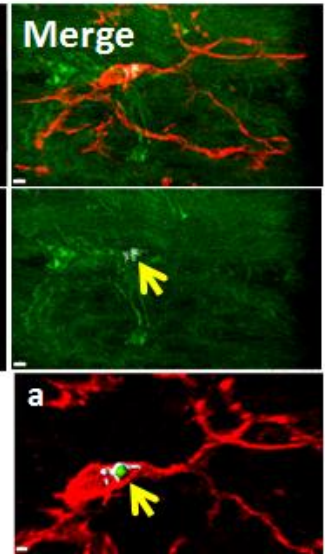

B

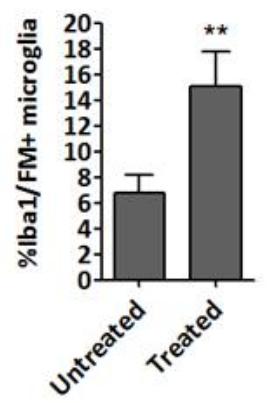

Figure 3.18 Myelin fragments are found within Lipofuscin in microglia in cuprizone mice $\mathbf{3 3}$ weeks after completed remyelination. A) Confocal image of lipofuscin (gray) within microglia (Iba1, red) containing myelin fragments (Fluoromyelin, green). Scale bars: $20 \mu \mathrm{m}$ (overview) and $2 \mu \mathrm{m}$ (zoom in). (a) 3-D reconstruction of lipofuscin (gray) including myelin (green) within microglia (red). Scale bar: $1 \mu \mathrm{m}$ B) Quantification of the number of lipofuscin positive cells that contain myelin. 6 treated and untreated (age-matched) mice were analyzed. Mean+SD was reported, ${ }^{* *} p<0.01$, Student's t-test.

\subsubsection{Clearance function of microglia in PMD mice}

We injected FITC-Dextran into the cortex of 7 months old PMD mice and quantified the number of microglia internalizing FITC-Dextran. Compared to age matched wild type mice, we found $50 \%$ reduction in clearance function of microglia in PMD mice (Fig 3.25) 


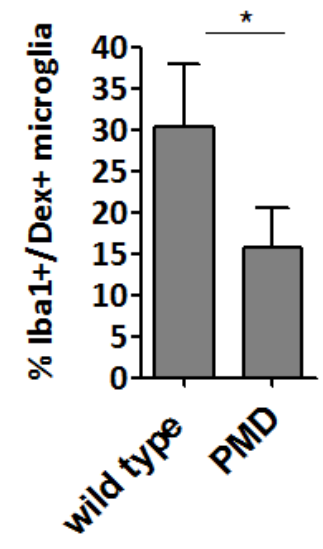

Figure 3.19 Quantification of Dextran uptake by microglia in 7-month-old PMD mice. Dextran-FITC was injected in the cortex of 7 months old PMD and wild type mice, 7 hours after injection the mice were analyzed to measure the percentage of Iba1/Dex positive microglia in the injection site. The number of these cells is significantly lower in PMD mice compared to wild type. Mean value+SD was reported, $n=4, * p<0.05$

\subsection{Genetically induced impairment of lysosomal degradation in microglia}

To analyze the effects of lysosomal storage on morphology and functionality of the cells we blocked the lysosomal degradation system specifically in microglia. This was achieved by crossing floxed Rab7 mice to a Tamoxifen inducible CX3CR1 Cre mouse line. Double transgenic mice $\left(\mathrm{CX} 3 \mathrm{CR}^{\mathrm{Cre+} /}\right.$; Rab7 $7^{\text {flox/flox }}$ ) were injected with Tamoxifen for 5 consecutive days to obtain conditional Rab7 knockout mice (Fig $3.20 \mathrm{~A}$ ). We started to analyze the conditional knockout mice at different time points after Tamoxifen injection (Fig 3.20 B).

\subsubsection{Cre recombinase-mediated deletion of Rab7 gene in conditional Rab7 knockout mice}

To evaluate deletion of Rab7 gene induced by Cre recombinase we performed two strategies as following. First, double transgenic mice were crossed to tdTomato reporter mice then $\mathrm{CX} 3 \mathrm{CR} 1^{+/ \text {CreERT2; }}$ Rab7 $7^{\text {flox/+ }}$; tdTomato+ mice were injected with Tamoxifen to induce Cre-mediated expression of tdTOmato in microglia. One week after Tamoxifen injection, histological studies showed that more than $95 \%$ of microglia in the brain were tdTomato positive, which indicated an efficient activity of cre recombinase in microglia (Fig $3.20 \mathrm{C}$ ). Second, one week after tamoxifen injection in CX3CR1 ${ }^{\mathrm{Cret} /}$; Rab7 $7^{\text {flox/flox }}$ mice, microglia cells were isolated to measure relative expression of Rab7 mRNA in the cells by quantitative real time PCR. The quantification showed a 98\% reduction of Rab7 transcript (Fig 3.20 D). 
A

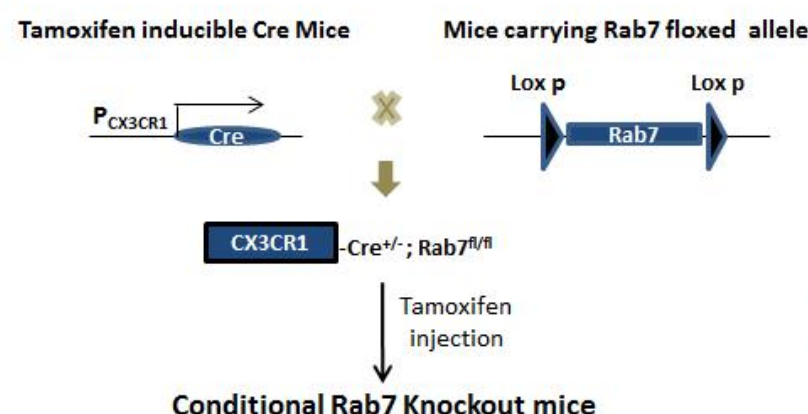

B

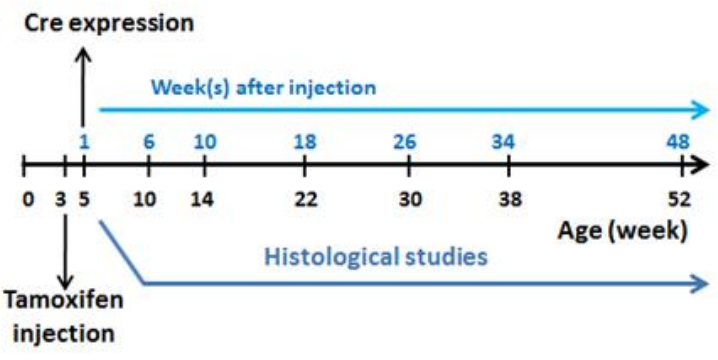

C
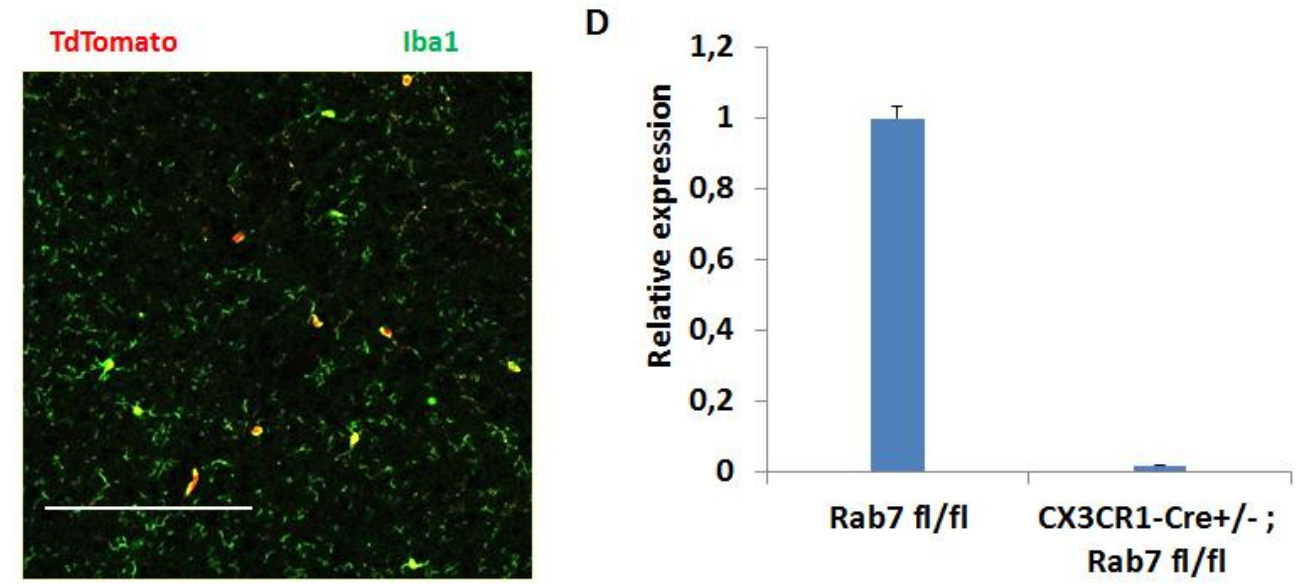

Figure 3.20 Evaluation of Rab7 gene deletion in conditional Rab7 knockout mice. A) Strategy for conditional targeting of Rab7 in mice. B) Timeline diagram for histoglogical analysis of conditional Rab7 knockout mice. C) Confocal image from cortex in CX3CR1 ${ }^{+/ C r e E R T 2 ; ~ R a b 7 ~} 7^{\text {flox/+}}$; tdTomato+ mice. One week after Tamoxifen injection, microglia were visualized with Iba1 in green and the number of Iba1+/tdTomato+ (red) cells were counted. The quantification showed that tdTomato was expressed in $95 \%$ of microglia, Scale bar: $300 \mu \mathrm{m}$. D) Relative expression of Rab7 mRNA in microglia one week after Tamoxifen injection in double transgenic mice (CX3CR1 ${ }^{\text {Cre+ } /-}$; Rab7 $7^{\text {flox/flox }}$ ). 98\% of Rab7 transcript was reduced.

\subsubsection{Phenotypic characterization of conditional Rab7 knockout mice}

\subsubsection{Enlarged lysosomes in microglia}

Considering the role of Rab7 in maturation of lysosomes, we first investigated the lysosomal morphology of microglia in Rab7 knockout mice. Brain sections were co-labeled with lba1 (against microglia) and LAMP1 (against lysosomes) using immunohistochemistry. Confocal images were captured to visualize lysosomes in microglia. We started our histological studies one week after Rab7 deletion when we didn't detect any changes in lysososmes within microglia. However, enlarged 
lysosomes appeared only 6 weeks after Rab7 deletion when the mice were 10 weeks old (Fig $3.21 \mathrm{~A}$ ). At this time point there was no detectable lysosomes in microglia of control group. Enlarged lysosomes were detectable in microglia of Rab7 knockout mice at later time points $(10,18$, and 26 weeks after Tamoxifen injection) as well. Quantification of the number of LAMP1 positive showed that almost $50 \%$ of microglia cells have enlarged lysosomes in conditional Rab7 knockout mice (Fig $3.21 \mathrm{~B})$.

A

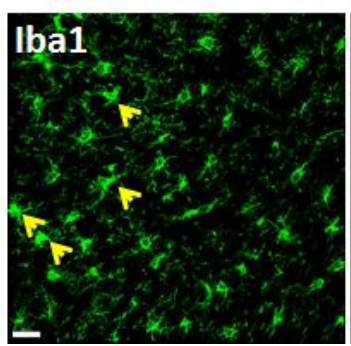

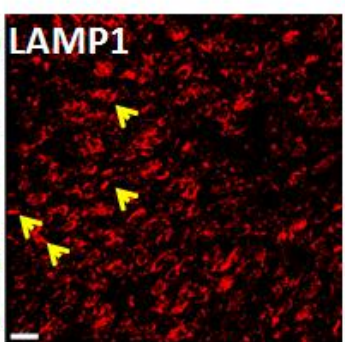

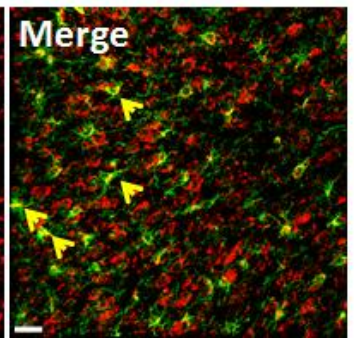

B

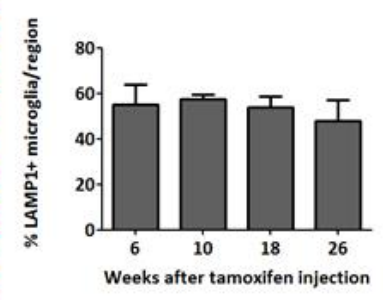

Figure 3.21 Visualization and quantification of LAMP1-positive microglia in conditional Rab7 knockout mice. A) Enlarged lysosomes (labeled with LAMP1 in red) are detectable within microglia (labeled with Iba1 in green) 6 weeks after Tamoxifen injection. Scale bar: $20 \mu \mathrm{m}$. B) The percentage of LAMP1-positive microglia cells in the cortical white matter in Rab7 knockout mice 6, 10, 18, and 26 weeks after Rab7 deletion.

\subsubsection{Myelin fragments inside microglia}

To study the interaction between microglia and myelin in conditional Rab7 KO mice, myelin and microglia were labeled with MBP and Iba1, respectively by immunohistochemistry. The labeled brain tissues of KO and control groups were analyzed 6, 10, 18, 26, 34, and 48 weeks after tamoxifen injection to detect myelin phagocytosis by microglia. Myelin fragments were detected inside microglia in KO mice 34weeks after Tamoxifen injection, when the mice were 9 months old (Fig 3.22 A). In wild type mice internalized myelin was detected only at 18 months of age (Section 3.2.2). The number of cells with internalized myelin components was quantified in $1 \mathrm{~mm}^{2}$ of white matter including corpus callosum and striatum. 2.78\% of microglia cells engulfed myelin. (Fig 3.22 B) 
A

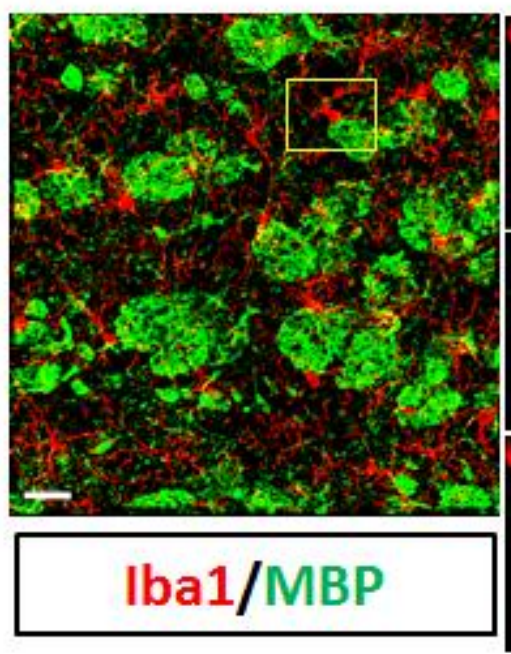

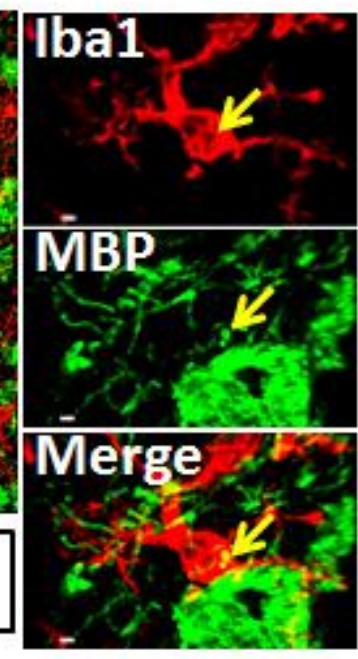

B

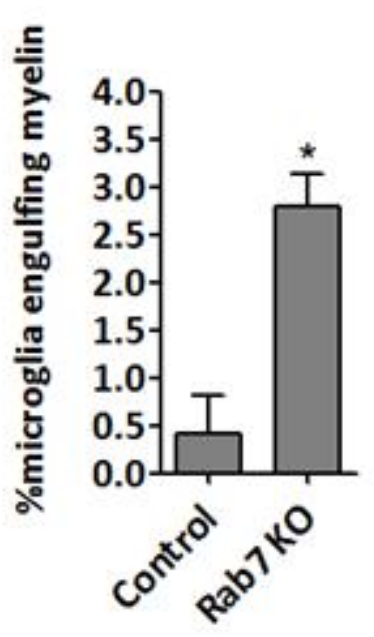

Figure 3.22 myelin phagocytosis by microglia in conditional Rab7 KO mice. A) Confocal image representing myelin components (MBP, green) within microglia (Iba1, red) in striatum of a 9month-old KO mouse (34 weeks after Tamoxifen injection). B) Quantification of the percentage of cells engulfing myelin fragments at the same time point. Each group: $n=3$, Mean+SD was indicated, ${ }^{*} p<0.05$, Student's t-test.

\subsubsection{Early accumulation of lipofuscin in microglia}

Immunohistological studies showed that a considerable amount of lipofuscin accumulated inside microglia in KO mice at very early time point ( 6 weeks after Tamoxifen injection) when the mice were only 10 weeks old. Quantification of the volume of lipofuscin 6, 18, 34, and 48 weeks after tamoxifen injection showed a significant increase in the amount of lipofuscin in KO mice in comparison with control group.

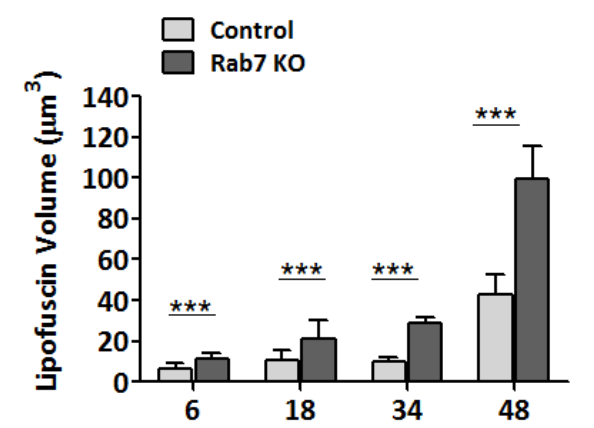

Weeks after tamoxifen injection
Figure 3.23 Lipofuscin in conditional Rab7 KO mice. The volume of lipofuscin in the cells was measured in the callosum and cortical white matter using Imaris software. The amount of lipofuscin in microglia in KO mice is significantly higher than that in control mice at all the time points. Three mice per group were analyzed and mean value plus $\mathrm{SD}$ is indicated. ${ }^{* * *} \mathrm{p}<0.0001$, Student' $\mathrm{t}$-test.

\subsubsection{Myelin fragments are associated with lipofuscin within microglia}

To search for myelin remnants within lipofuscin, myelin was labeled with MBP and Fluoromyelin. Myelin and lipofuscin were visualized simultaneously using dye separation method with confocal 
microscopy as explained before. We found MBP or Fluoromyelin positive lipofuscin granules 34 and 48 weeks after Tamoxifen injection (Fig 3.24).

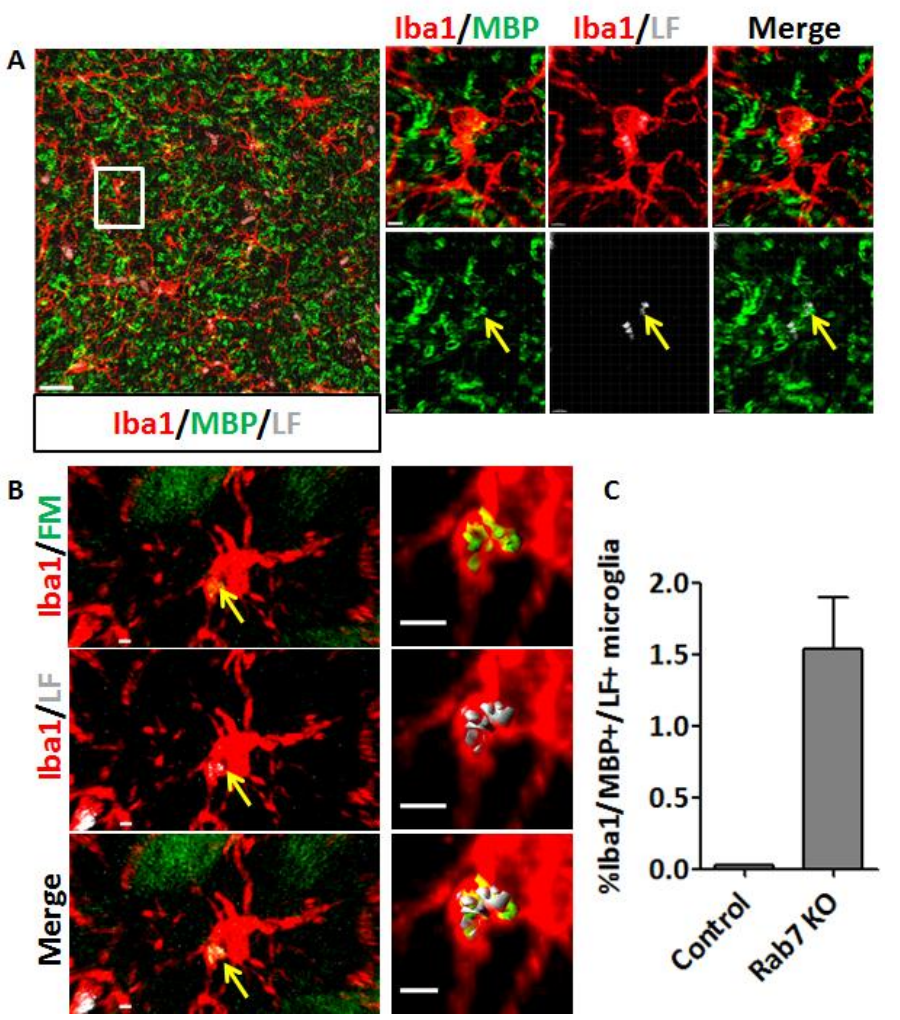

Figure $\quad 3.24 \quad$ Lipofuscin contains internalized myelin fragments in microglia in Rab7 KO mice. A) 48 weeks after Tamoxifen injection when the mice were 12 months old, myelin fragments (MBP, green) were detected inside microglia (Iba1, red) while they were associated with intracellular lipofuscin (LF, gray). Scale bar: $20 \mu \mathrm{m}$ (overview) and $2 \mu \mathrm{m}$ (zoom in). B) 3-dimensional reconstruction showing Fluoromyelinlabeled myelin fragment (green) within lipofuscin (gray) inside a single microglia cell Scale bar: $2 \mu \mathrm{m} \mathrm{C)}$ Quantification of the number of cells with internalized MBP associated with lipofuscin.

\subsubsection{Clearance function of microglia in conditional Rab7 KO mice}

Due to early accumulation of lipofuscin as well as lysosomal dysfunction in microglia in Rab7 KO mice we assumed that the clearance function of the cells might be impaired. FITC-Dextran was injected into the cortex of KO and control mice 6 and 18 weeks after Tamoxifen injection. Clearance function of microglia in KO mice was significantly reduced only at the later time point (18 weeks after Tamoxifen injection) while there was no significant difference between control and KO mice at earlier time point, 6 weeks after Tamoxifen injection (Fig 3.25) 


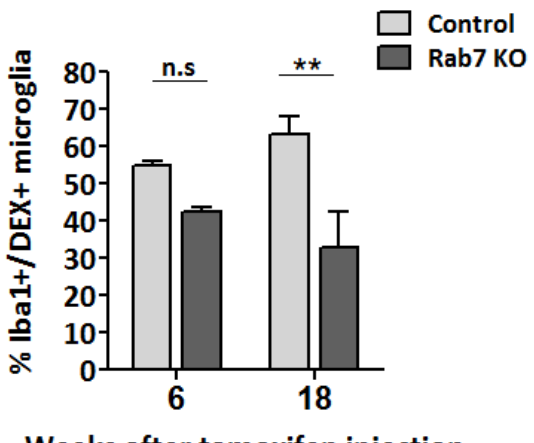

Weeks after tamoxifen injection
Figure 3.25 Clearance function of microglia in conditional Rab7 KO mice. Dextran was injected into the cortex 6 and 18 weeks after Tamoxifen injection; 7 hours after injection, the number of Iba1/Dex+ cells was counted. There was no difference in clearance function of cells between $\mathrm{KO}$ and control mice 6 weeks after Tamoxifen injection. There was a $50 \%$ reduction in the number of Dextran positive microglia in $\mathrm{KO}$ mice in comparison to control group at later time point (18 weeks after Tamoxifen injection). $n=4$, mean+SD, ${ }^{* *} p$ value $<0.01$, n.s: $p=0.3206$, Student's t-test.

\subsubsection{Myelin fragments in conditional Rab7 KO mice}

We quantified the number of myelin fragments in electron microscopic images from white matter, mainly corpus callosum, in $\mathrm{KO}$ and control mice 48 and 77 weeks after Tamoxifen injection. At both time points there was a significant increase in the number of extracellular myelin fragments (Fig 3.26).

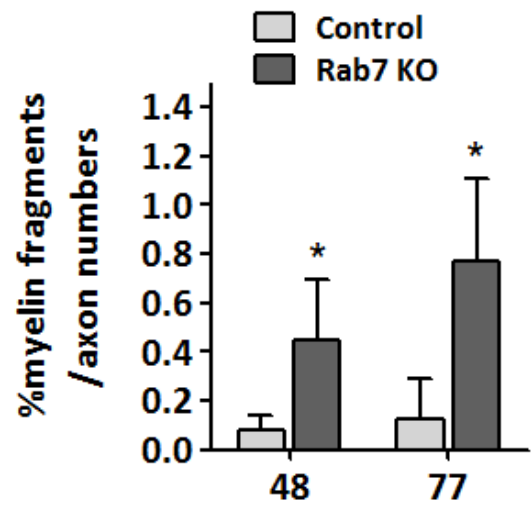

Weeks after tamoxifen injection
Figure 3.26 Number of myelin fragments increases in conditional Rab7 KO mice. Brain tissues from control and KO mice 48 and 77 weeks after Tamoxifen injection were prepared for conventional electron microscopy. The number of myelin fragments was counted per 1000 axons. The quantification shows that myelin fragments in KO mice are significantly more than control groups at both time points.

\subsubsection{Microglia morphology in conditional Rab7 KO mice}

Microglia were analyzed in Rab7 KO mice morphologically using immunohistochemistry. Compared to control group, microglia in KO mice showed smaller and less complex processes 6 weeks after tamoxifen injection (Fig 3.27 A). In addition, the surface area occupied by microglia processes in KO mice was reduced compared to control mice (Fig $3.27 \mathrm{~B}$ ). Although the KO mice at this time point were only 10 weeks old, the morphology of microglia was comparable to those of aged microglia. 
Control

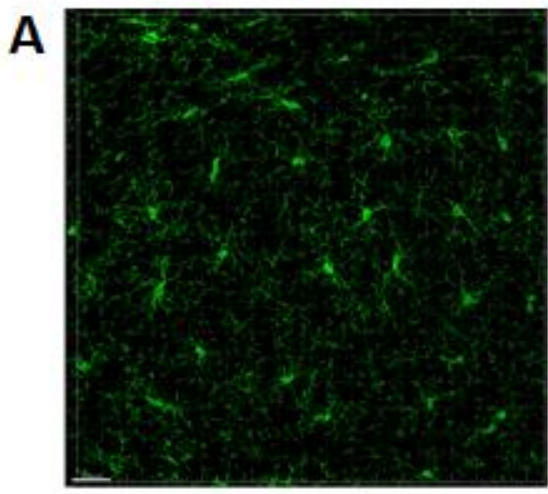

Rab7 KO

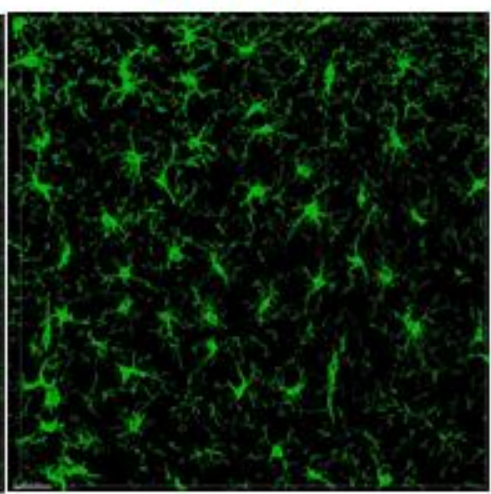

B

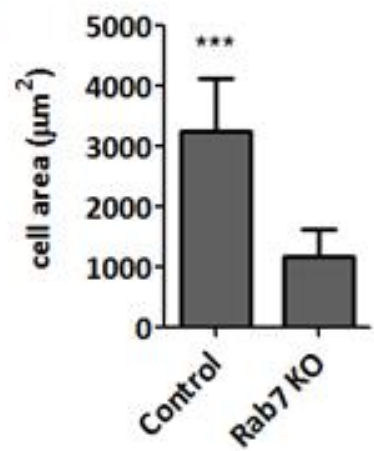

Figure 3.27 morphological analysis of microglia in Rab7 KO mice. A) Confocal image of microglia population in cortical white matter 6 weeks after Tamoxifen injection showing shorter and less branched processes in KO compared to control mice. Scale bar: $30 \mu \mathrm{m}$. B) Quantification shows that cell area of microglia in KO mice is significantly less than cell area in control group. ${ }^{* * *} p<0.0001$, Mean value+SD.

\subsubsection{MHCll expression in microglia in conditional Rab7 KO mice}

To assess the activation state of microglia in KO mice, using DAB histostaining, we evaluated expression of Mac2, Mac3, MHCII, CR3(CD11b/ Mac1), Fcy RII/III, at different time points (18, 26, 34, 48 and 77 weeks after Tamoxifen injection). The results showed that there were no Mac3 positive cells in any of the mice, and there was no significant difference in number of CR3 and Fcy RII/III positive cells between $\mathrm{KO}$ and control mice $26,34,48$, and 77 weeks after Tamoxifen injection. In addition, Mac2 and $\mathrm{MHCll}$ were not expressed in both $\mathrm{KO}$ and control mice 18, 26, and 34 weeks after Tamoxifen injection. The number of MHCII positive cells in KO mice 48 and 77 weeks after Tamoxifen injection was significantly higher compared to control group.

A
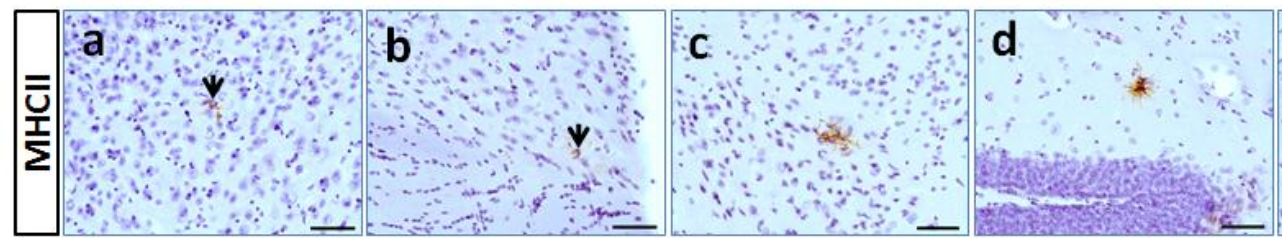

ev

B

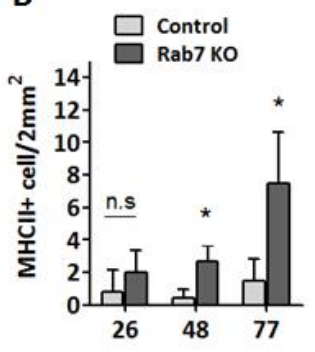

weeks after tamoxifen injection
Figure 3.28 $\mathrm{MHCll}$ expression in microglia of Rab7 KO mice. A) A few MHCll positive cells were detected in Rab7 KO mice 48 weeks after Tamoxifen injection in cortical gray matter (a), cortical white matter (b), striatum (c), hippocampus (d) and corpus callosum (e). Scale bar: $50 \mu \mathrm{m}$. B) Quantification of MHCll positive cell in Rab7 KO and control mice 26, 48, and 77 weeks after Tamoxifen injection showing a significant increase in $\mathrm{KO}$ mice at later time points compared to their control group. $n=3$, Mean plus SD was represented, ${ }^{*} p<0.05$, n.s: $p=0,2221$ 


\section{Chapter 4}

\section{Discussion}

\subsection{Myelin turnover by shedding of myelin fragments into the extracellular space}

Myelin is a tightly packed membrane in which the protein and lipid molecules are sandwiched between compacted layers. This special membrane has been considered as one of the most stable membranes. Myelin molecules have little contact with the metabolic pool in the cytoplasm so that they exhibit a low rate of metabolic turnover (O'Brien 1965). Previously, the turnover rate of some myelin lipids and proteins were determined using isotope-labeling and mass spectrometry (MS). Sphingomyelin, gangeliosides, cerebroside, and cholesterol were found to have low rate of metabolism. Proteolipid protein (PLP) with the half-life of 20-35 days was reported as metabolically active myelin component (Smith 1968; Fischer and Morell 1974; Ando, Tanaka et al. 2003). The incorporation of tyrosine into the myelin proteins using [14C] tyrosine showed that only $20 \%$ of myelin proteins were replaced within 10 days (Lajtha, Toth et al. 1977)

Due to the heterogeneity of myelin structure, the turnover rates of myelin lipids and proteins are heterogeneous. (Smith 1968; D'Monte, Mela et al. 1971; Sabri, Bone et al. 1974; Poduslo and Braun 1975; Figlewicz and Druce 1976; Lajtha, Toth et al. 1977; Singh and Jungalwala 1979). Myelin proteins that have access to the metabolic machinery might have higher turnover rate (Lajtha, Toth et al. 1977). Iodination studies showed that MBP is less exposed to the outer surface compared to PLP (Poduslo and Braun 1975). Recently, using pulse-chase labeling and mass spectrometry, myelin proteins such as MBP, PLP, and MOG were reported as long-lived myelin proteins (Toyama, Savas et al. 2013).

Long-lived proteins are at increased risk for accumulation within the cells which can be linked to the cellular aging process (Toyama, Savas et al. 2013). Therefore, structures like myelin membrane exhibiting long-term protein persistence need to be maintained or remodeled. Studies in human and rodents claimed that myelin is continuously exchanged (Lasiene, Matsui et al. 2009; Young, Psachoulia et al. 2013; Yeung, Zdunek et al. 2014). Studies in human brain suggested that preexisting mature oligodendrocytes are responsible for myelin modulation. Oligodendrocytes in human show a very low rate of turnover. After 9 years of age the number of oligodendrocytes remains stable throughout the rest of the human lifespan. There is no correlation between oligodendrocyte 


\section{4 |Discussion}

turnover and myelin remodeling that occurs at a high rate in human. However, several observations in the rodents indicate that oligodendrocyte turnover contributes to myelin remodeling (Dimou, Simon et al. 2008; Emery 2010; Young, Psachoulia et al. 2013). 30\% of all oligodendrocytes in the corpus callosum in adult mice are newly differentiated (Rivers, Young et al. 2008; Zhu, Whittemore et al. 2011). Adult oligodendrocytes are thought to be required for the maintenance of myelin by the replacement or remodeling of preexisting myelin sheaths (Young, Psachoulia et al. 2013). For the remodeling of myelin, new myelin segment appears to displace old myelin segments (Wang and Young 2014). Internode retraction and expansion of the node of Ranvier due to disruption of key proteins lead to a transient hypofusion. This mechanism is suggested to play a role in myelin remodeling (Reimer, McQueen et al. 2011).

It still remains to be understood how the old segments of myelin sheath are removed from axons. How the molecules that are trapped within closely compacted membranes get access to the degradation system for myelin turnover during long period of time. Using electron microscopy, we detected multilamellar myelin fragments in the intracellular and extracellular space in adult and aged mice. We noticed that a large part of myelin sheath tears away from the axon (Fig $4.1 \mathrm{~A}$ ). In addition, light microscopic histology in the striatum, interestingly, showed that a part of myelin is pulled by microglia in aged mice (Fig 4.1 B). We propose that myelin fragments can be formed by shedding of myelin sheaths into the extracellular space.

A

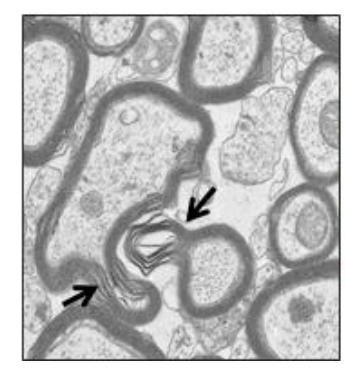

B

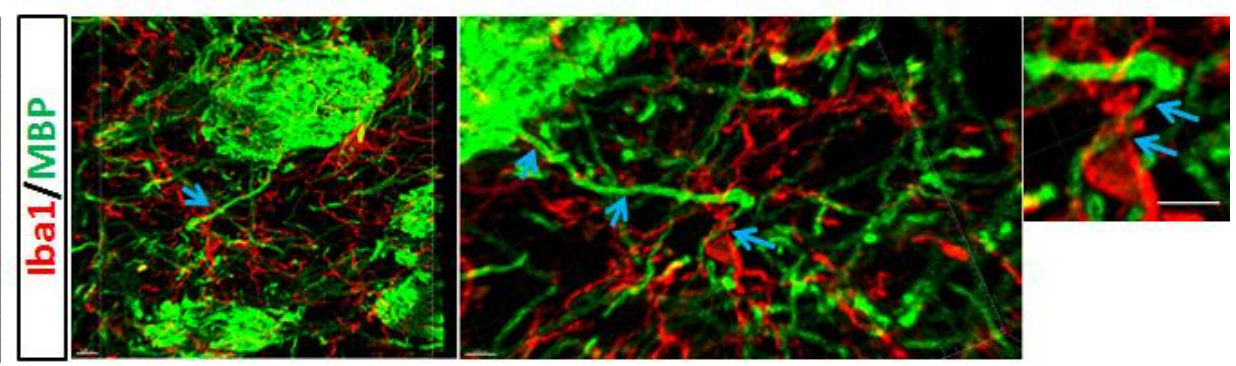

Figure 4. 1 Myelin fragments are formed by shedding of myelin sheaths into the extracellular space A) Elecron microscopic image in the corpus callosum showing myelin tearing away from the axon. B) Confocal image in striatum of 18 months old mouse showing a microglia (Iba1, red) pulling a part of myelin (MBP, green) into the extracellular space. The middle image shows the same phenomenon in a rotated position. Scale bar, $5 \mu \mathrm{m}$ (left); $4 \mu \mathrm{m}$ (middle and right).

\subsection{Myelin uptake by microglia}

The processes of microglia in their "resting" state in vivo are highly motile to survey the microenvironment in the CNS and also to interact with synapses (Nimmerjahn, Kirchhoff et al. 2005; Wake, Moorhouse et al. 2009). We speculated that resting microglia have also potential functions in myelin turnover in the CNS. Our work showed that microglia processes are in contact with myelin in 
physiological condition. The frequency of these contacts and also the surface area in contact with myelin increase with age, starting at least at 9 months of age. In addition, myelin fragments were detected inside microglia cells in striatum as well as cortical white matter at 18 months of age and later. Consistent with previous observations, we found an age-dependent increase in the number of microglia cells in the white matter (Sandell and Peters 2002). Moreover, compared to gray matter, a considerable number of cells expressing Mac2/Galectine 3 were observed in the white matter. Interestingly, this population was detectable only in aged mice. Mac2/Galectine 3 is a galactosebinding lectin which appears to be active in demyelination of peripheral nerves (Saada, Reichert et al. 1996). Mac2/Galectine 3 is also known to be upregulated in demyelination areas in EAE, and is expressed by microglia, macrophages that phagocytose myelin (Reichert and Rotshenker 1999; Smith 2001; Rotshenker, Reichert et al. 2008; Rotshenker 2009). Additionally, while lysosomes were rarely detectable in young microglia, they appear from 7 months of age on. Also, in both middle aged and aged mice, lysosomes are strikingly larger in the white matter than in gray matter. This finding suggests that the lysosomal system of microglia cells is actively engaged in degradation of myelin. Previous studies have shown an increase in the number of CD68 positive microglia cells which are involved in the engulfment of synaptic elements in the postnatal retinogeniculate system. CD68 is a marker for lysosomes (Schafer, Lehrman et al. 2012). Moreover, our results are consistent with previous work that has shown an increase in the number of ED1, a lysosomal marker, positive microglia in corpus callosum with age (Hua, Schindler et al. 2012) Altogether, our results indicate that microglia cells are actively involved in the removal of myelin in normal aging.

\subsection{Age-related increase in microglia-myelin contact is correlated with higher rate of myelin turnover in aging}

Electron microscopic studies have revealed that during aging myelin sheaths undergo some structural alterations that make the membrane unstable and prone to degeneration (Peters 2002). In this context, it has been shown that in aged mice the replacement of some molecules such as cerebroside and GM1 is relatively accelerated and metabolic turnover of myelin is slightly higher (Ando, Tanaka et al. 2003). Previous studies on oligodendrocytes in aged brain of monkeys have detected dense inclusions which aggregate in the cell body or develop swellings at the end of processes (Peters 2002). These inclusions are suggested to originate from degenerating myelin membranes and turnover of material in the sheaths. In some oligodendrocytes, the swelling was seen along the length of their processes (LeVine and Torres 1992). 


\section{4 | Discussion}

To maintain the integrity of myelin, unstable molecules must be removed either from axons or from oligodendrocytes. Accordingly, our studies show that microglia can be responsible for clearing degenerated myelin in aged brain of mice. Our results specifically shows that myelin molecules such as MBP which are trapped within layers of tightly compacted membrane are transferred to microglia by fragmentation of myelin in the extracellular space. The other possibility is that myelin components are outsourced by oligodendrocytes into the extracellular space.

In adulthood, myelin is remodeled or replaced by new myelin sheaths that are produced by adultborn oligodendrocytes (Wang and Young 2014). These cells, intrinsically, generate shorter myelin segments not only in adulthood but also in aging to actively myelinate the axons which have not been myelinated in development (Lasiene, Matsui et al. 2009; Young, Psachoulia et al. 2013). These studies suggest that myelination continues from development to adulthood as well as aging. Therefore, the old segments from pre-existing myelin sheaths during remodeling of myelin membrane as well as myelin disposal generated due to de novo myelination must be removed efficiently for the maintenance of myelinated fibers.

\subsection{Internalized myelin contribute to the formation of lysosomal inclusions}

The normal functionality of microglia cells is essential for the integrity of the CNS in healthy as well as pathological conditions. During the life span of an animal, microglia cells are targeted with the aging that cause changes in their morphology and functions (Ajami, Bennett et al. 2007; Streit and Xue 2010). Therefore, it is important to define the factors which induce age-related alterations in these cells.

Consistent with other studies we could also detect an aging phenotype in microglia in wild type mice. One of the most specific hallmarks of aging in microglia is lipofuscin accumulation, defined as lysosomal inclusions (Sierra, Gottfried-Blackmore et al. 2007; Tremblay, Zettel et al. 2012). There was an age-related increase in the number and amount of intracellular lipofuscin. It is notable that lipofuscin was not totally absent from cells in young ( 2 months old) mouse brain, but was detectable only in very small amount. Formation of lipofuscin even in the early time of life is thought to be due to oxidative reactions which are important for the survival of the cell during the lifetime (Terman and Brunk 1998). In middle aged and aged mice, lipofuscin positive microglia cells were found all over the brain. However, interestingly, microglia in the white matter have larger amount of lipofuscin compared to gray matter. In addition, we found that internalized myelin fragments are frequently 
associated with lipofuscin. The main reason for lipofuscin formation is thought to be age-related and (reactive oxygen species) ROS-mediated oxidative stress. Endogenous ROS which is mainly derived from mitochondria cause oxidative damage to the internalized material in lysosomes so that they become undegradable and form lipofuscin (Terman and Brunk 1998; Terman, Kurz et al. 2010). Therefore, lipofuscin is considered as a mixture of oxidized proteins and lipids (Gray and Woulfe 2005). Myelin proteins and lipids are known to be susceptible to ROS-induced oxidation (Bongarzone, Pasquini et al. 1995; Luoma, Kuo et al. 2015). This type of oxidation is mainly mediated by iron which increases with age and accumulates within lipofuscin (Brunk 1989; Gray and Woulfe 2005; Wong 2013). Myelin membrane exposure to iron-catalyzed free radical generation system induces protein aggregation and lipoperoxidation (Konat, Gantt et al. 1986; Domanska-Janik and Bourre 1990). Altogether, we suggest that myelin components which are engulfed by microglia with time undergo oxidative damage so that accumulate within the cell as a part of lipofuscin.

\subsection{Microglial phenotype changes with age}

We observed that lipofuscin positive cells have shorter and retracted branches, and are less motile. Other studies have also shown that aged microglia cells in the brain as well as retina have less branched processes, reduced arborization and motility (Sierra, Gottfried-Blackmore et al. 2007; Damani, Zhao et al. 2011). We found a correlation between the presence of intracellular lipofuscin and ramification as well as motility of their processes. The latter characteristics of microglia are required for the surveillance function. Because of less dynamic and ramified processes of aged microglia, the cellular migration of cells toward a local lesion is not as efficient as in young cells. Consequently, their immune responses may become slower. Moreover, following injury, aged cells represent a delayed dynamics of deactivation; they are not dispersed from the lesion site after a long time (Damani, Zhao et al. 2011). Sustained accumulation of activated aged cells avoids the maintenance of tissue homeostasis and may induce chronic neuroinflammation involved in the pathogenesis of age-related neurodegenerative diseases (Medzhitov 2008; Damani, Zhao et al. 2011). Furthermore, it is known that the aggregation of undigested materials within the cells leads to deficient clearance function. For example, oxidized lipoprotein-loaded macrophages, known as foamy cells in atherosclerosis, are inefficient in clearance of apoptotic bodies (Schrijvers, De Meyer et al. 2007; Ley, Miller et al. 2011). We expected that aged microglia with accumulation of lipofuscin, a mixture of oxidized proteins and lipids, also show an impaired clearance function. Following injection of Dextran-FITC in the cortex of young (2-month-old) and middle aged (10-month-old) mice, we could see a reduction in dextran uptake by 10-month-old mice. Consistent with the other studies (Frank, 


\section{4 | Discussion}

Barrientos et al. 2006). Overall, we suggest that lipofuscin can be considered as a biomarker of senescent and dysfunctional microglia.

\subsection{Myelin breakdown leads to lipofuscin formation}

In summary, our results show that in aging brain, myelin is fragmented and taken up by microglia where it is partially included within lipofuscin, and that lipofuscin positive cells represent a senescence-type of phenotype. We asked whether there is a casual correlation between myelin uptake by microglia and their aging phenotype. We assumed that overloading the lysosomal system with myelin may induce microglia senescence. Therefore, we analyzed microglia in demyelinating mouse models in which the cells are exposed to large amount of myelin fragments due to enhanced myelin breakdown. Immunohistochemical analysis of dysmyelinating mouse model for PelizaeusMerzbacher disease, which overexpress plp 1 gene (PMD mice), at different age, $(2,7$, and 10 months old) showed that the stability of myelin is reduced with time and consequently, myelin sheaths were broken-down. Loss of myelin was accompanied with accumulation of microglia expressing $\mathrm{MHCI}$ and Mac2 in the white matter. These observations are in consistent with the studies that characterized these mice previously (Karim, Barrie et al. 2007; Edgar, McCulloch et al. 2010; Tatar, Appikatla et al. 2010). We frequently found myelin fragments within these cells at later time when demyelination was peaking (10 months of age). In addition, by comparing transgenic mice at different age we detected a significant increase in the amount of intracellular lipofuscin with time. There was no difference between PMD and wild type mice at 2 months of age when the myelin was still stable. Compared to age-matched wild type mice there was a striking increase in the quantity of lipofuscin in 7 as well as 10 months old PMD mice in which the stability of myelin sheaths was compromised. The difference between 10 months old PMD mice and wild type was bigger than difference between 7 months old PMD mice and wild type. Altogether, these data showed that the accumulation of lipofuscin overlaps with the level of myelin breakdown in these mice.

Next, we observed that microglia in 7 months old PMD mice have a reduced capacity to clear away dextran particles from the extracellular space. Therefore, considering the high level of myelin fragmentation in these mice we suggest that overwhelming the cells with myelin fragments and consequently, lipofuscin affects their clearance function.

Next to demyelination, PMD mice develop axonal degeneration due to inflammation and oligodendrocyte dysfunction (Anderson, Schneider et al. 1998; Edgar, McCulloch et al. 2010). Thus, in these mice the formation of inclusions in microglia may results from engulfment of not only myelin 
but also axonal elements. Therefore, to avoid the contribution of other factors besides myelin, we used shiverer mice in which there is no sign of axonal change or loss and the pathology is restricted to myelin (Griffiths, Klugmann et al. 1998). In these mice, myelin breakdown occurs due to lack of MBP already in development (Roach, Takahashi et al. 1985). Previous studies have reported that there is no macrophage infiltration in shiverer mice (Bird, Farrell et al. 1978). However, we could detect a massive accumulation of microglia in the corpus callosum in these mice at P25 as well as P63. These cells have a significant amount of lipofuscin indicating that myelin degeneration contributes to its accumulation.

In these models, demyelination or dysmyelination are continuous processes. We asked whether a short-term exposure of myelin to the cells would be also sufficient to enhance aging at a later time. To test this, we induce a singular event of demyelination for 4 weeks by cuprizone treatment of wild type mice, and then induce remyelination by changing cuprizone to the normal diet. According to previous studies (Matsushima and Morell 2001; McMahon, Suzuki et al. 2002), microglia remain in their activation state 4-6 weeks after removing cuprizone from the diet. Thus, we started our histological studies 4 weeks after diet change, when remylination was completed, to differentiate phenotypic changes that might occur as a result of activation from those produced as a consequence of aging. As the main hallmark of aging we quantified the volume of lipofuscin in microglia cells at later time points after completed remyelination. In comparison with the control mice, the amount of lipofuscin increased significantly in cuprizone-treated mice already 5 weeks after completed remyelination and continued to increase 11 and 33 weeks after completed remyelination. In addition, interestingly we could find myelin fragments associated with lipofuscin even 33 weeks after complete remyelination, confirming our previous data that showed the association of internalized myelin with lipofuscin in wild type conditions.

Further investigation of cuprizone mice showed that $\mathrm{MHCll}$ and Mac2 were up-regulated in corpus callosum 5 weeks after completed remyelination. These up-regulations were expected as an indication of microglia activation in this time point. Interestingly, while Mac2 positive cells were still detectable 11, 19, and 33 weeks after complete remyelination, $\mathrm{MHCll}$ expression went back to the basal level and reappeared only at week 33 after complete remyelination. These data indicate that low grade inflammation which is normally characteristic of microglia senescence occurs earlier when the brain has undergone a single event of widespread demyelination. Moreover, expression of Mac2 at all the time points can be explained regarding its role in myelin phagocytosis during demyelination (Smith 1999; Smith 2001). Notably, at the latest time point in cuprizone mice Mac2 expressing cells were found mainly in anterior commissure, and previously in aged wild type mice we also detected the same population of microglia in the same region of brain. Therefore, we propose that even in 


\section{4 | Discussion}

normal aging the contact of microglia with myelin fragments, produced due to myelin sheaths breakdown, keeps the cells in a mild activation state that mainly appears as Mac2 expression. Studies on demyelinating models, including PMD, shiverers, and cuprizone mice, suggest that myelin breakdown can accelerate age-related phenotype in microglia cells.

Myelin consists of long-lived proteins (Lajtha, Toth et al. 1977; Ando, Tanaka et al. 2003; Toyama, Savas et al. 2013). Long-lived proteins are thought to have implications for age-dependent deficiency in the cells and tissues. These components are possibly at the risk of damage during a long period of time (Toyama, Savas et al. 2013). For example, it was shown that accumulation of damaged crystalline with time cause vision deficiency (Bloemendal, de Jong et al. 2004; Wilmarth, Tanner et al. 2006). Long-lived scaffold nucleoporins undergo oxidative damage during aging that is associated with defects in nuclear trafficking and the breakdown of the nuclear permeability barrier (D'Angelo, Raices et al. 2009). Due to the nature of myelin components, they are thought to be vulnerable to the iron-catalyzed proxidation occurring normally in lysosome for degradation (Bongarzone, Pasquini et al. 1995; Luoma, Kuo et al. 2015). Therefore, these components become indigestible with time. The constant contact of non-dividing cells such as microglia with myelin cause lysosomal storage which can be linked to deficient clearance function (Schrijvers, De Meyer et al. 2007) and possibly age-related features.

\subsection{Lysosomal storage induces cellular senescence}

We asked how microglia senescence is induced. We could show that when the cells are exposed to myelin fragments or overloaded with them, the amount of intracellular lipofuscin increases and premature aging phenotype is induced. Therefore, we suggested that overloading the lysosomal system with time may influence the functionality of microglia and induce cellular senescence. To test this, we generated conditional Rab7 Knockout mice in which the cargo transport from endosomes to lysosomes is blocked so that a pile of material in degradative compartments accumulates within microglia. In these mice, enlarged lysosomes, as an indication of lysosomal dysfunction, were detectable in the cells only 6 weeks after Tamoxifen injection and not earlier than that although just one week after Tamoxifen injection more than 98\% of Rab7 transcript was already deleted. We assumed that at the earlier time point, residual pool of Rab7 protein in the cytosol still can compensate the functionality of lysosomes in the cells. It is notable that the enlarged lysosomes were detectable for 48 weeks after Tamoxifen injection which shows stability of microglia in the CNS (Lawson, Perry et al. 1992) 
Interestingly, the hallmarks of senescence, including lipofuscin accumulation and shorter and less branched cell processes, were detected in microglia in Rab7 KO mice already when the mice were 10 weeks old. Moreover, regardless the normal aging effect, at later time points, the amount of lipofuscin significantly increased in Rab7 KO mice in comparison with control mice. The clearance function of cells decreased significantly with time. Possibly, due to higher amount of indigestible materials, lysosomes are more exhausted at later time so that the cells are not efficient enough for clearance. These data are comparable with our previous results that showed less clearance activity in aged cells with high amount of lipofuscin, and also with other studies shown that lysosomal storage in phagocytic cells cause a defect in clearance of apoptotic bodies (Schrijvers, De Meyer et al. 2007). Due to deficient clearance function of microglia cells in Rab7 KO mice a higher number of extracellular myelin fragments in the white matter was found confirming that microglia cells are responsible for myelin fragments removal in aging.

At the age of one year a few cells expressing MHCll were detected while there was no positive cell in control mice. Altogether, the results show that impairment of lysosomal degradation system induces a pre-mature aging phenotype in microglia. We propose that there is a reciprocal correlation between aging and lyososomal function. While aging factors, mainly oxidative stress, affects the functionality of the lysosomes (Kurz, Terman et al. 2008), dysfunctional lysosomes may induce cellular aging.

We found that Rab7 KO mice develop intracellular MBP positive myelin fragments more frequently compared to control group. Furthermore, myelin uptake by microglia in Rab7 KO mice was detected 9 months earlier than in wild type. The internalized myelin fragments were found in association with lipofuscin more abundant in KO mice. Overall, using this mouse model we could confirm our previous results showing that in normal aging myelin fragments are constantly taken up by microglia and partially get trapped within intracellular lipofuscin.

\section{8 conclusion}

In summary, we suggest that myelin breakdown plays a role in inducing microglia dysfunctionality that occurs in the aging brain. Increasing rate of myelin turnover in demyelinating models as well as in normal aging cause cells to become overloaded with myelin fragments with time, and develop lysosomal storage pathology, which consequently induce aging.

Our results may have important implication for neurodegenerative disease. If constant transfer of myelin fragments may block the lysosomal system and consequently impairs the function of microglia cells in a long term, this may be relevant for diseases associated with protein aggregation. 


\section{4 | Discussion}

For example, in Alzheimer's disease, it is known that the capacity of microglia to remove $\beta$-Amyloid goes down with time.

Our findings may explain why prior traumatic brain injury (TBI) associated with tissue breakdown acts as a risk factor for chronic neurodegenerative diseases. We propose that a long term exposure to myelin debris or neuronal elements cause microglia cells to be overloaded so that they become exhausted and may lose their function to clean brain from more debris.

Furthermore, our results may also be important for the pathophysiology of CNS autoimmune diseases such as multiple sclerosis. In this disease, myelin is known to be the most common target of autoimmune responses (Steinman and Zamvil 2003; Fletcher, Lalor et al. 2010). However, according to immunopathology of MS, it is not well understood how myelin antigens reach antigen presenting cells in the CNS to reactivate auto-reactive T cells in the parenchyma (Goverman 2009; Hart, Hintzen et al. 2009). We suggest that the permanent circulation of myelin-derived components in the brain parenchyma may predispose myelin towards autoimmune diseases. 


\section{Bibliography}

Adamec, E., P. S. Mohan, et al. (2000). "Up-regulation of the lysosomal system in experimental models of neuronal injury: implications for Alzheimer's disease." Neuroscience 100(3): 663-675.

Aggarwal, S., L. Yurlova, et al. (2011). "Central nervous system myelin: structure, synthesis and assembly." Trends Cell Biol 21(10): 585-593.

Ajami, B., J. L. Bennett, et al. (2007). "Local self-renewal can sustain CNS microglia maintenance and function throughout adult life." Nat Neurosci 10(12): 1538-1543.

Alliot, F., I. Godin, et al. (1999). "Microglia derive from progenitors, originating from the yolk sac, and which proliferate in the brain." Brain Res Dev Brain Res 117(2): 145-152.

Anderson, T. J., A. Schneider, et al. (1998). "Late-onset neurodegeneration in mice with increased dosage of the proteolipid protein gene." J Comp Neurol 394(4): 506-519.

Ando, S., Y. Tanaka, et al. (2003). "Turnover of myelin lipids in aging brain." Neurochem Res 28(1): 513.

Bachstetter, A. D., J. M. Morganti, et al. (2011). "Fractalkine and CX 3 CR1 regulate hippocampal neurogenesis in adult and aged rats." Neurobiol Aging 32(11): 2030-2044.

Bakhti, M., S. Aggarwal, et al. (2014). "Myelin architecture: zippering membranes tightly together." Cell Mol Life Sci 71(7): 1265-1277.

Banati, R. B. (2003). "Neuropathological imaging: in vivo detection of glial activation as a measure of disease and adaptive change in the brain." Br Med Bull 65: 121-131.

Barden, H. (1980). "Interference filter microfluorometry of neuromelanin and lipofuscin in human brain." J Neuropathol Exp Neurol 39(5): 598-605.

Baumann, N. and D. Pham-Dinh (2001). "Biology of oligodendrocyte and myelin in the mammalian central nervous system." Physiol Rev 81(2): 871-927.

Bhaskar, K., M. Konerth, et al. (2010). "Regulation of tau pathology by the microglial fractalkine receptor." Neuron 68(1): 19-31.

Biber, K., H. Neumann, et al. (2007). "Neuronal 'On' and 'Off' signals control microglia." Trends Neurosci 30(11): 596-602.

Bird, T. D., D. F. Farrell, et al. (1978). "Brain lipid composition of the shiverer mouse: (genetic defect in myelin development)." J Neurochem 31(1): 387-391.

Blakemore, W. F. (1972). "Observations on oligodendrocyte degeneration, the resolution of status spongiosus and remyelination in cuprizone intoxication in mice." J Neurocytol 1(4): 413-426. 


\section{Bibliography}

Bloemendal, H., W. de Jong, et al. (2004). "Ageing and vision: structure, stability and function of lens crystallins." Prog Biophys Mol Biol 86(3): 407-485.

Bok, D. (1993). "The retinal pigment epithelium: a versatile partner in vision." J Cell Sci Suppl 17: 189195.

Bongarzone, E. R., J. M. Pasquini, et al. (1995). "Oxidative damage to proteins and lipids of CNS myelin produced by in vitro generated reactive oxygen species." J Neurosci Res 41(2): 213-221.

Boulton, M., M. Rozanowska, et al. (2004). "The photoreactivity of ocular lipofuscin." Photochem Photobiol Sci 3(8): 759-764.

Bradl, M., J. Bauer, et al. (1999). "Transgenic Lewis rats overexpressing the proteolipid protein gene: myelin degeneration and its effect on T cell-mediated experimental autoimmune encephalomyelitis." Acta Neuropathol 97(6): 595-606.

Brunk, U. T. (1989). "On the origin of lipofuscin; the iron content of residual bodies, and the relation of these organelles to the lysosomal vacuome. A study on cultured human glial cells." Adv Exp Med Biol 266: 313-320; discussion 321-312.

Brunk, U. T. and A. Terman (2002). "Lipofuscin: mechanisms of age-related accumulation and influence on cell function." Free Radic Biol Med 33(5): 611-619.

Bucci, C., R. G. Parton, et al. (1992). "The small GTPase rab5 functions as a regulatory factor in the early endocytic pathway." Cell 70(5): 715-728.

Bucci, C., P. Thomsen, et al. (2000). "Rab7: a key to lysosome biogenesis." Mol Biol Cell 11(2): 467480.

Bunge, M. B., R. P. Bunge, et al. (1962). "Electron microscopic demonstration of connections between glia and myelin sheaths in the developing mammalian central nervous system." J Cell Biol 12: 448453.

Butovsky, O., M. P. Jedrychowski, et al. (2014). "Identification of a unique TGF-beta-dependent molecular and functional signature in microglia." Nat Neurosci 17(1): 131-143.

Campagnoni, A. T. and W. B. Macklin (1988). "Cellular and molecular aspects of myelin protein gene expression." Mol Neurobiol 2(1): 41-89.

Chavrier, P., R. G. Parton, et al. (1990). "Localization of low molecular weight GTP binding proteins to exocytic and endocytic compartments." Cell 62(2): 317-329.

Chernoff, G. F. (1981). "Shiverer: an autosomal recessive mutant mouse with myelin deficiency." 지 Hered 72(2): 128.

Chrast, R., G. Saher, et al. (2011). "Lipid metabolism in myelinating glial cells: lessons from human inherited disorders and mouse models." J Lipid Res 52(3): 419-434.

Clarke, L. E., K. M. Young, et al. (2012). "Properties and fate of oligodendrocyte progenitor cells in the corpus callosum, motor cortex, and piriform cortex of the mouse." J Neurosci 32(24): 8173-8185. 
Conde, J. R. and W. J. Streit (2006). "Microglia in the aging brain." J Neuropathol Exp Neurol 65(3): 199-203.

Corral-Debrinski, M., T. Horton, et al. (1992). "Mitochondrial DNA deletions in human brain: regional variability and increase with advanced age." Nat Genet 2(4): 324-329.

D'Angelo, M. A., M. Raices, et al. (2009). "Age-dependent deterioration of nuclear pore complexes causes a loss of nuclear integrity in postmitotic cells." Cell 136(2): 284-295.

D'Monte, B., P. Mela, et al. (1971). "Metabolic instability of myelin protein and proteolipid fractions." Eur J Biochem 23(2): 355-365.

Damani, M. R., L. Zhao, et al. (2011). "Age-related alterations in the dynamic behavior of microglia." Aging Cell 10(2): 263-276.

Dimou, L., C. Simon, et al. (2008). "Progeny of Olig2-expressing progenitors in the gray and white matter of the adult mouse cerebral cortex." J Neurosci 28(41): 10434-10442.

Domanska-Janik, K. and J. M. Bourre (1990). "Effect of lipid peroxidation on Na+,K(+)-ATPase, 5'nucleotidase and CNPase in mouse brain myelin." Biochim Biophys Acta 1034(2): 200-206.

Dowson, J. H. (1982). "The evaluation of autofluorescence emission spectra derived from neuronal lipopigment." J Microsc 128(Pt 3): 261-270.

Dowson, J. H., D. Armstrong, et al. (1982). "Autofluorescence emission spectra of neuronal lipopigment in animal and human ceroidoses (ceroid-lipofuscinoses)." Acta Neuropathol 58(2): 152156.

Duncan, I. D., J. P. Hammang, et al. (1987). "Abnormal compact myelin in the myelin-deficient rat: absence of proteolipid protein correlates with a defect in the intraperiod line." Proc Natl Acad Sci U S A 84(17): 6287-6291.

Edgar, J. M., M. C. McCulloch, et al. (2010). "Demyelination and axonal preservation in a transgenic mouse model of Pelizaeus-Merzbacher disease." EMBO Mol Med 2(2): 42-50.

Emery, B. (2010). "Regulation of oligodendrocyte differentiation and myelination." Science 330(6005): 779-782.

Essner, E. and A. B. Novikoff (1960). "Human hepatocellular pigments and lysosomes." J Ultrastruct Res 3: 374-391.

Evans, T. A., D. S. Barkauskas, et al. (2014). "High-resolution intravital imaging reveals that bloodderived macrophages but not resident microglia facilitate secondary axonal dieback in traumatic spinal cord injury." Exp Neurol 254: 109-120.

Feeney-Burns, L. and G. E. Eldred (1983). "The fate of the phagosome: conversion to 'age pigment' and impact in human retinal pigment epithelium." Trans Ophthalmol Soc U K 103 ( Pt 4): 416-421.

Fields, R. D. (2008). "White matter in learning, cognition and psychiatric disorders." Trends Neurosci 31(7): 361-370. 


\section{Bibliography}

Figlewicz, D. A. and M. J. Druce (1976). "Incorporation of (3H)leucine into three subfractions of myelin in murine myelin dysgenesis: the quaking mutant." J Neurochem 26(3): 625-627.

Finnemann, S. C., L. W. Leung, et al. (2002). "The lipofuscin component A2E selectively inhibits phagolysosomal degradation of photoreceptor phospholipid by the retinal pigment epithelium." Proc Natl Acad Sci U S A 99(6): 3842-3847.

Fischer, C. A. and P. Morell (1974). "Turnover of proteins in myelin and myelin-like material of mouse brain." Brain Res 74(1): 51-65.

Fitzner, D., M. Schnaars, et al. (2011). "Selective transfer of exosomes from oligodendrocytes to microglia by macropinocytosis." J Cell Sci 124(Pt 3): 447-458.

Flanary, B. E., N. W. Sammons, et al. (2007). "Evidence that aging and amyloid promote microglial cell senescence." Rejuvenation Res 10(1): 61-74.

Flanary, B. E. and W. J. Streit (2005). "Effects of axotomy on telomere length, telomerase activity, and protein in activated microglia." J Neurosci Res 82(2): 160-171.

Fletcher, J. M., S. J. Lalor, et al. (2010). "T cells in multiple sclerosis and experimental autoimmune encephalomyelitis." Clin Exp Immunol 162(1): 1-11.

Frank, M. G., R. M. Barrientos, et al. (2006). "mRNA up-regulation of MHC II and pivotal proinflammatory genes in normal brain aging." Neurobiol Aging 27(5): 717-722.

Funato, K., W. Beron, et al. (1997). "Reconstitution of phagosome-lysosome fusion in streptolysin Opermeabilized cells." J Biol Chem 272(26): 16147-16151.

Ginhoux, F., M. Greter, et al. (2010). "Fate mapping analysis reveals that adult microglia derive from primitive macrophages." Science 330(6005): 841-845.

Ginhoux, F., S. Lim, et al. (2013). "Origin and differentiation of microglia." Front Cell Neurosci 7: 45.

Gledhill, R. F. and W. I. McDonald (1977). "Morphological characteristics of central demyelination and remyelination: a single-fiber study." Ann Neurol 1(6): 552-560.

Gomes-Leal, W. (2012). "Microglial physiopathology: how to explain the dual role of microglia after acute neural disorders?" Brain Behav 2(3): 345-356.

Gomez-Nicola, D. and V. H. Perry (2015). "Microglial dynamics and role in the healthy and diseased brain: a paradigm of functional plasticity." Neuroscientist 21(2): 169-184.

Goverman, J. (2009). "Autoimmune T cell responses in the central nervous system." Nat Rev Immunol 9(6): 393-407.

Gray, D. A. and J. Woulfe (2005). "Lipofuscin and aging: a matter of toxic waste." Sci Aging Knowledge Environ 2005(5): re1.

Griffiths, I., M. Klugmann, et al. (1998). "Axonal swellings and degeneration in mice lacking the major proteolipid of myelin." Science 280(5369): 1610-1613. 
Gurkan, C., H. Lapp, et al. (2005). "Large-scale profiling of Rab GTPase trafficking networks: the membrome." Mol Biol Cell 16(8): 3847-3864.

Harry, G. J. (2013). "Microglia during development and aging." Pharmacol Ther 139(3): 313-326.

Hart, A. D., A. Wyttenbach, et al. (2012). "Age related changes in microglial phenotype vary between CNS regions: grey versus white matter differences." Brain Behav Immun 26(5): 754-765.

Hasegawa-Ishii, S., S. Takei, et al. (2011). "Morphological impairments in microglia precede agerelated neuronal degeneration in senescence-accelerated mice." Neuropathology 31(1): 20-28.

Heneka, M. T., M. P. Kummer, et al. (2014). "Innate immune activation in neurodegenerative disease." Nat Rev Immunol 14(7): 463-477.

Hildebrand, C., S. Remahl, et al. (1993). "Myelinated nerve fibres in the CNS." Prog Neurobiol 40(3): 319-384.

Hiremath, M. M., Y. Saito, et al. (1998). "Microglial/macrophage accumulation during cuprizoneinduced demyelination in C57BL/6 mice." J Neuroimmunol 92(1-2): 38-49.

Hoffmann, K., M. Lindner, et al. (2008). "Epileptic seizures and hippocampal damage after cuprizoneinduced demyelination in C57BL/6 mice." Exp Neurol 210(2): 308-321.

Hohn, A. and T. Grune (2013). "Lipofuscin: formation, effects and role of macroautophagy." Redox Biol 1: 140-144.

Hua, K., M. K. Schindler, et al. (2012). "Regionally distinct responses of microglia and glial progenitor cells to whole brain irradiation in adult and aging rats." PLoS One 7(12): e52728.

Hutagalung, A. H. and P. J. Novick (2011). "Role of Rab GTPases in membrane traffic and cell physiology." Physiol Rev 91(1): 119-149.

Iqbal, K., C. Alonso Adel, et al. (2005). "Tau pathology in Alzheimer disease and other tauopathies." Biochim Biophys Acta 1739(2-3): 198-210.

Iqbal, K., I. Grundke-lqbal, et al. (1989). "Identification and localization of a tau peptide to paired helical filaments of Alzheimer disease." Proc Natl Acad Sci U S A 86(14): 5646-5650.

Jung, S., J. Aliberti, et al. (2000). "Analysis of fractalkine receptor CX(3)CR1 function by targeted deletion and green fluorescent protein reporter gene insertion." Mol Cell Biol 20(11): 4106-4114.

Kaarniranta, K., D. Sinha, et al. (2013). "Autophagy and heterophagy dysregulation leads to retinal pigment epithelium dysfunction and development of age-related macular degeneration." Autophagy 9(7): 973-984.

Kaemmerer, E., F. Schutt, et al. (2007). "Effects of lipid peroxidation-related protein modifications on RPE Iysosomal functions and POS phagocytosis." Invest Ophthalmol Vis Sci 48(3): 1342-1347.

Kang, S. H., M. Fukaya, et al. (2010). "NG2+ CNS glial progenitors remain committed to the oligodendrocyte lineage in postnatal life and following neurodegeneration." Neuron 68(4): 668-681. 


\section{Bibliography}

Karim, S. A., J. A. Barrie, et al. (2007). "PLP overexpression perturbs myelin protein composition and myelination in a mouse model of Pelizaeus-Merzbacher disease." Glia 55(4): 341-351.

Karlsson, U. and R. L. Schultz (1965). "Fixation of the central nervous system from electron microscopy by Aldehyde perfusion. I. Preservetion with Aldehyde perfusates versus direct perfusion with osmium tetroxide with special reference to membranes and the extracellular space." I Ultrastruct Res 12: 160-186.

Katz, M. L. (2002). "Potential role of retinal pigment epithelial lipofuscin accumulation in age-related macular degeneration." Arch Gerontol Geriatr 34(3): 359-370.

Kesterson, J. W. and W. W. Carlton (1971). "Histopathologic and enzyme histochemical observations of the cuprizone-induced brain edema." Exp Mol Pathol 15(1): 82-96.

Kettenmann, H., U. K. Hanisch, et al. (2011). "Physiology of microglia." Physiol Rev 91(2): 461-553.

Komoly, S., M. D. Jeyasingham, et al. (1987). "Decrease in oligodendrocyte carbonic anhydrase activity preceding myelin degeneration in cuprizone induced demyelination." J Neurol Sci 79(1-2): 141-148.

Konat, G., G. Gantt, et al. (1986). "Peroxidative aggregation of myelin membrane proteins." Metab Brain Dis 1(3): 177-185.

Kornfeld, S. and I. Mellman (1989). "The biogenesis of lysosomes." Annu Rev Cell Biol 5: 483-525.

Kosik, K. S., C. L. Joachim, et al. (1986). "Microtubule-associated protein tau (tau) is a major antigenic component of paired helical filaments in Alzheimer disease." Proc Natl Acad Sci U S A 83(11): 40444048.

Kurz, T., A. Terman, et al. (2008). "Lysosomes and oxidative stress in aging and apoptosis." Biochim Biophys Acta 1780(11): 1291-1303.

Lajtha, A., J. Toth, et al. (1977). "Turnover of myelin proteins in mouse brain in vivo." Biochem J 164(2): 323-329.

Lasiene, J., A. Matsui, et al. (2009). "Age-related myelin dynamics revealed by increased oligodendrogenesis and short internodes." Aging Cell 8(2): 201-213.

Lawson, L. J., V. H. Perry, et al. (1990). "Heterogeneity in the distribution and morphology of microglia in the normal adult mouse brain." Neuroscience 39(1): 151-170.

Lawson, L. J., V. H. Perry, et al. (1992). "Turnover of resident microglia in the normal adult mouse brain." Neuroscience 48(2): 405-415.

Lee, C. Y. and G. E. Landreth (2010). "The role of microglia in amyloid clearance from the AD brain." J Neural Transm 117(8): 949-960.

Letiembre, M., W. Hao, et al. (2007). "Innate immune receptor expression in normal brain aging." Neuroscience 146(1): 248-254.

LeVine, S. M. and M. V. Torres (1992). "Morphological features of degenerating oligodendrocytes in twitcher mice." Brain Res 587(2): 348-352. 
Ley, K., Y. I. Miller, et al. (2011). "Monocyte and macrophage dynamics during atherogenesis." Arterioscler Thromb Vasc Biol 31(7): 1506-1516.

Lin, M. T., D. K. Simon, et al. (2002). "High aggregate burden of somatic mtDNA point mutations in aging and Alzheimer's disease brain." Hum Mol Genet 11(2): 133-145.

Lombardi, D., T. Soldati, et al. (1993). "Rab9 functions in transport between late endosomes and the trans Golgi network." EMBO J 12(2): 677-682.

Lourbopoulos, A., A. Erturk, et al. (2015). "Microglia in action: how aging and injury can change the brain's guardians." Front Cell Neurosci 9: 54.

Luo, X. G., J. Q. Ding, et al. (2010). "Microglia in the aging brain: relevance to neurodegeneration." Mol Neurodegener 5: 12.

Luoma, A. M., F. Kuo, et al. (2015). "Plasmalogen phospholipids protect internodal myelin from oxidative damage." Free Radic Biol Med 84: 296-310.

Lutcke, A., R. G. Parton, et al. (1994). "Cloning and subcellular localization of novel rab proteins reveals polarized and cell type-specific expression." J Cell Sci 107 (Pt 12): 3437-3448.

Ma, W., S. Coon, et al. (2013). "A2E accumulation influences retinal microglial activation and complement regulation." Neurobiol Aging 34(3): 943-960.

March, P. A., S. Wurzelmann, et al. (1995). "Morphological alterations in neocortical and cerebellar GABAergic neurons in a canine model of juvenile Batten disease." Am J Med Genet 57(2): 204-212.

Matsushima, G. K. and P. Morell (2001). "The neurotoxicant, cuprizone, as a model to study demyelination and remyelination in the central nervous system." Brain Pathol 11(1): 107-116.

Matthews, M. A. and D. Duncan (1971). "A quantitative study of morphological changes accompanying the initiation and progress of myelin production in the dorsal funiculus of the rat spinal cord." J Comp Neurol 142(1): 1-22.

McCoy, M. K., K. A. Ruhn, et al. (2008). "Intranigral lentiviral delivery of dominant-negative TNF attenuates neurodegeneration and behavioral deficits in hemiparkinsonian rats." Mol Ther 16(9): $1572-1579$.

McMahon, E. J., K. Suzuki, et al. (2002). "Peripheral macrophage recruitment in cuprizone-induced CNS demyelination despite an intact blood-brain barrier." J Neuroimmunol 130(1-2): 32-45.

Medzhitov, R. (2008). "Origin and physiological roles of inflammation." Nature 454(7203): 428-435.

Mellman, I., R. Fuchs, et al. (1986). "Acidification of the endocytic and exocytic pathways." Annu Rev Biochem 55: 663-700.

Merkler, D., S. Boretius, et al. (2005). "Multicontrast MRI of remyelination in the central nervous system." NMR Biomed 18(6): 395-403.

Mildner, A., H. Schmidt, et al. (2007). "Microglia in the adult brain arise from Ly-6ChiCCR2+ monocytes only under defined host conditions." Nat Neurosci 10(12): 1544-1553. 
Miller, K. R. and W. J. Streit (2007). "The effects of aging, injury and disease on microglial function: a case for cellular senescence." Neuron Glia Biol 3(3): 245-253.

Mouton, P. R., J. M. Long, et al. (2002). "Age and gender effects on microglia and astrocyte numbers in brains of mice." Brain Res 956(1): 30-35.

Murray, J. A. and W. F. Blakemore (1980). "The relationship between internodal length and fibre

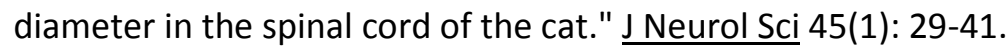

Nakanishi, H., Y. Hayashi, et al. (2011). "The role of microglial mtDNA damage in age-dependent prolonged LPS-induced sickness behavior." Neuron Glia Biol 7(1): 17-23.

Nave, K. A. (2010). "Myelination and support of axonal integrity by glia." Nature 468(7321): 244-252.

Neufeld, E. F. (1991). "Lysosomal storage diseases." Annu Rev Biochem 60: 257-280.

Neuroscience Center, U. o. N. C. a. C. H. (1999). Basic Neurochemistry: Molecular, Cellular and Medical Aspects. Myelin Formation, Structure and Biochemistry, American Society for Neurochemistry.

Nimmerjahn, A., F. Kirchhoff, et al. (2005). "Resting microglial cells are highly dynamic surveillants of brain parenchyma in vivo." Science 308(5726): 1314-1318.

Njie, E. G., E. Boelen, et al. (2012). "Ex vivo cultures of microglia from young and aged rodent brain reveal age-related changes in microglial function." Neurobiol Aging 33(1): 195 e191-112.

Noelker, C., L. Morel, et al. (2013). "Toll like receptor 4 mediates cell death in a mouse MPTP model of Parkinson disease." Sci Rep 3: 1393.

Norden, D. M. and J. P. Godbout (2013). "Review: microglia of the aged brain: primed to be activated and resistant to regulation." Neuropathol Appl Neurobiol 39(1): 19-34.

Nowotny, K., T. Jung, et al. (2014). "Accumulation of modified proteins and aggregate formation in aging." Exp Gerontol 57: 122-131.

Nukina, N. and Y. Ihara (1985). "Proteolytic fragments of Alzheimer's paired helical filaments." $\underline{\mathrm{J}}$ Biochem 98(6): 1715-1718.

O'Brien, J. S. (1965). "STABILITY OF THE MYELIN MEMBRANE." Science 147(3662): 1099-1107.

Ogura, K., M. Ogawa, et al. (1994). "Effects of ageing on microglia in the normal rat brain: immunohistochemical observations." Neuroreport 5(10): 1224-1226.

Parkhurst, C. N., G. Yang, et al. (2013). "Microglia promote learning-dependent synapse formation through brain-derived neurotrophic factor." Cell 155(7): 1596-1609.

Pattison, I. H. and J. N. Jebbett (1971). "Clinical and histological observations on cuprizone toxicity and scrapie in mice." Res Vet Sci 12(4): 378-380.

Peinado, M. A., A. Quesada, et al. (1998). "Quantitative and ultrastructural changes in glia and pericytes in the parietal cortex of the aging rat." Microsc Res Tech 43(1): 34-42. 
Pereira-Leal, J. B. and M. C. Seabra (2001). "Evolution of the Rab family of small GTP-binding proteins." J Mol Biol 313(4): 889-901.

Perry, V. H. (1998). "A revised view of the central nervous system microenvironment and major histocompatibility complex class II antigen presentation." J Neuroimmunol 90(2): 113-121.

Perry, V. H. and C. Holmes (2014). "Microglial priming in neurodegenerative disease." Nat Rev Neurol 10(4): 217-224.

Perry, V. H., J. A. Nicoll, et al. (2010). "Microglia in neurodegenerative disease." Nat Rev Neurol 6(4): 193-201.

Perry, V. H. and J. Teeling (2013). "Microglia and macrophages of the central nervous system: the contribution of microglia priming and systemic inflammation to chronic neurodegeneration." Semin Immunopathol 35(5): 601-612.

Peters, A. (2002). "The effects of normal aging on myelin and nerve fibers: A review." Journal of Neurocytology 31: 581-593.

Peters, A. (2007). "The Effects of Normal Aging on Nerve Fibers and Neuroglia in the Central Nervous System."

Peters, A. and T. Kemper (2012). "A review of the structural alterations in the cerebral hemispheres of the aging rhesus monkey." Neurobiol Aging 33(10): 2357-2372.

Peters, A. and C. Sethares (2003). "Is there remyelination during aging of the primate central nervous system?" J Comp Neurol 460(2): 238-254.

Pfeffer, S. and D. Aivazian (2004). "Targeting Rab GTPases to distinct membrane compartments." Nat Rev Mol Cell Biol 5(11): 886-896.

Pfeiffer, S. E., A. E. Warrington, et al. (1993). "The oligodendrocyte and its many cellular processes." Trends Cell Biol 3(6): 191-197.

Poduslo, J. F. and P. E. Braun (1975). "Topographical arrangement of membrane proteins in the intact myelin sheath. Lactoperoxidase incorproation of iodine into myelin surface proteins." J Biol Chem 250(3): 1099-1105.

Poliak, S. and E. Peles (2003). "The local differentiation of myelinated axons at nodes of Ranvier." Nat Rev Neurosci 4(12): 968-980.

Poliani, P. L., Y. Wang, et al. (2015). "TREM2 sustains microglial expansion during aging and response to demyelination." J Clin Invest 125(5): 2161-2170.

Ponomarev, E. D., L. P. Shriver, et al. (2005). "Microglial cell activation and proliferation precedes the onset of CNS autoimmunity." J Neurosci Res 81(3): 374-389.

Prinz, M. and J. Priller (2014). "Microglia and brain macrophages in the molecular age: from origin to neuropsychiatric disease." Nat Rev Neurosci 15(5): 300-312. 


\section{Bibliography}

Prinz, M., J. Priller, et al. (2011). "Heterogeneity of CNS myeloid cells and their roles in neurodegeneration." Nat Neurosci 14(10): 1227-1235.

Privat, A., C. Jacque, et al. (1979). "Absence of the major dense line in myelin of the mutant mouse "shiverer"." Neurosci Lett 12(1): 107-112.

Psachoulia, K., F. Jamen, et al. (2009). "Cell cycle dynamics of NG2 cells in the postnatal and ageing brain." Neuron Glia Biol 5(3-4): 57-67.

Quarles, P. M. a. R. H. (1999). Basic Neurochemistry: Molecular, Cellular and Medical Aspects. Chapter 4 Myelin Formation, Structure and Biochemistry. S. GJ. Philadelphia, Lippincott-Raven.

Readhead, C. and L. Hood (1990). "The dysmyelinating mouse mutations shiverer (shi) and myelin deficient (shimld)." Behav Genet 20(2): 213-234.

Reichert, F. and S. Rotshenker (1999). "Galectin-3/MAC-2 in experimental allergic encephalomyelitis." Exp Neurol 160(2): 508-514.

Reimer, M. M., J. McQueen, et al. (2011). "Rapid disruption of axon-glial integrity in response to mild cerebral hypoperfusion." J Neurosci 31(49): 18185-18194.

Rink, J., E. Ghigo, et al. (2005). "Rab conversion as a mechanism of progression from early to late endosomes." Cell 122(5): 735-749.

Rivers, L. E., K. M. Young, et al. (2008). "PDGFRA/NG2 glia generate myelinating oligodendrocytes and piriform projection neurons in adult mice." Nat Neurosci 11(12): 1392-1401.

Roach, A., N. Takahashi, et al. (1985). "Chromosomal mapping of mouse myelin basic protein gene and structure and transcription of the partially deleted gene in shiverer mutant mice." Cell 42(1): 149-155.

Rogers, J., J. Luber-Narod, et al. (1988). "Expression of immune system-associated antigens by cells of the human central nervous system: relationship to the pathology of Alzheimer's disease." Neurobiol Aging 9(4): 339-349.

Rosenbluth, J. (1981). "Axoglial junctions in the mouse mutant Shiverer." Brain Res 208(2): 283-297.

Rotshenker, S. (2009). "The role of Galectin-3/MAC-2 in the activation of the innate-immune function of phagocytosis in microglia in injury and disease." J Mol Neurosci 39(1-2): 99-103.

Rotshenker, S., F. Reichert, et al. (2008). "Galectin-3/MAC-2, Ras and PI3K activate complement receptor-3 and scavenger receptor-Al/II mediated myelin phagocytosis in microglia." Glia 56(15): 1607-1613.

Saada, A., F. Reichert, et al. (1996). "Granulocyte macrophage colony stimulating factor produced in lesioned peripheral nerves induces the up-regulation of cell surface expression of MAC-2 by macrophages and Schwann cells." J Cell Biol 133(1): 159-167.

Sabri, M. I., A. H. Bone, et al. (1974). "Turnover of myelin and other structural proteins in the developing rat brain." Biochem J 142(3): 499-507. 
Salter, M. W. and S. Beggs (2014). "Sublime microglia: expanding roles for the guardians of the CNS." Cell 158(1): 15-24.

Salzer, J. L. (2003). "Polarized domains of myelinated axons." Neuron 40(2): 297-318.

Sandell, J. H. and A. Peters (2001). "Effects of age on nerve fibers in the rhesus monkey optic nerve." J Comp Neurol 429(4): 541-553.

Sandell, J. H. and A. Peters (2002). "Effects of age on the glial cells in the rhesus monkey optic nerve." J Comp Neurol 445(1): 13-28.

Sandhir, R., G. Onyszchuk, et al. (2008). "Exacerbated glial response in the aged mouse hippocampus following controlled cortical impact injury." Exp Neurol 213(2): 372-380.

Santos, A. M., R. Calvente, et al. (2008). "Embryonic and postnatal development of microglial cells in the mouse retina." J Comp Neurol 506(2): 224-239.

Satoh, J., Y. B. Lee, et al. (1995). "T-cell costimulatory molecules B7-1 (CD80) and B7-2 (CD86) are expressed in human microglia but not in astrocytes in culture." Brain Res 704(1): 92-96.

Schafer, D. P., E. K. Lehrman, et al. (2012). "Microglia sculpt postnatal neural circuits in an activity and complement-dependent manner." Neuron 74(4): 691-705.

Schnell, S. A., W. A. Staines, et al. (1999). "Reduction of lipofuscin-like autofluorescence in fluorescently labeled tissue." J Histochem Cytochem 47(6): 719-730.

Schrijvers, D. M., G. R. De Meyer, et al. (2007). "Phagocytosis in atherosclerosis: Molecular mechanisms and implications for plaque progression and stability." Cardiovasc Res 73(3): 470-480.

Seitelberger, F. (1995). "Neuropathology and genetics of Pelizaeus-Merzbacher disease." Brain Pathol 5(3): 267-273.

Sheng, J. G., R. E. Mrak, et al. (1998). "Enlarged and phagocytic, but not primed, interleukin-1 alphaimmunoreactive microglia increase with age in normal human brain." Acta Neuropathol 95(3): 229234.

Sierra, A., A. C. Gottfried-Blackmore, et al. (2007). "Microglia derived from aging mice exhibit an altered inflammatory profile." Glia 55(4): 412-424.

Singh, H. and F. B. Jungalwala (1979). "The turnover of myelin proteins in adult rat brain." Int J Neurosci 9(2): 123-131.

Sitte, N., M. Huber, et al. (2000). "Proteasome inhibition by lipofuscin/ceroid during postmitotic aging of fibroblasts." FASEB J 14(11): 1490-1498.

Smith, M. E. (1968). "The turnover of myelin in the adult rat." Biochim Biophys Acta 164(2): 285-293.

Smith, M. E. (1999). "Phagocytosis of myelin in demyelinative disease: a review." Neurochem Res 24(2): 261-268. 


\section{Bibliography}

Snaidero, N., W. Mobius, et al. (2014). "Myelin membrane wrapping of CNS axons by PI(3,4,5)P3dependent polarized growth at the inner tongue." Cell 156(1-2): 277-290.

Sparkman, N. L., L. A. Martin, et al. (2005). "Effects of intraperitoneal lipopolysaccharide on Morris maze performance in year-old and 2-month-old female C57BL/6J mice." Behav Brain Res 159(1): 145151.

Sparrow, J. R. and M. Boulton (2005). "RPE lipofuscin and its role in retinal pathobiology." Exp Eye Res 80(5): 595-606.

Sparrow, J. R. and T. Duncker (2014). "Fundus Autofluorescence and RPE Lipofuscin in Age-Related Macular Degeneration." J Clin Med 3(4): 1302-1321.

Steinman, L. and S. Zamvil (2003). "Transcriptional analysis of targets in multiple sclerosis." Nat Rev Immunol 3(6): 483-492.

Stenmark, H. and V. M. Olkkonen (2001). "The Rab GTPase family." Genome Biol 2(5): REVIEWS3007.

Strauss, O. (2005). "The retinal pigment epithelium in visual function." Physiol Rev 85(3): 845-881.

Strehler, B. L., D. D. Mark, et al. (1959). "GEE MV: Rate and magnitude of age pigment accumulation in the human myocardium." J Gerontol 14: 430-439.

Streit, W. J., N. W. Sammons, et al. (2004). "Dystrophic microglia in the aging human brain." Glia 45(2): 208-212.

Streit, W. J. and Q. S. Xue (2009). "Life and death of microglia." J Neuroimmune Pharmacol 4(4): 371379.

Streit, W. J. and Q. S. Xue (2010). "The Brain's Aging Immune System." Aging Dis 1(3): 254-261.

t Hart, B. A., R. Q. Hintzen, et al. (2009). "Multiple sclerosis - a response-to-damage model." Trends Mol Med 15(6): 235-244.

Tatar, C. L., S. Appikatla, et al. (2010). "Increased Plp1 gene expression leads to massive microglial cell activation and inflammation throughout the brain." ASN Neuro 2(4): e00043.

Terman, A., N. Abrahamsson, et al. (1999). "Ceroid/lipofuscin-loaded human fibroblasts show increased susceptibility to oxidative stress." Exp Gerontol 34(6): 755-770.

Terman, A. and U. T. Brunk (1998). "Lipofuscin: mechanisms of formation and increase with age." APMIS 106(2): 265-276.

Terman, A., T. Kurz, et al. (2010). "Mitochondrial turnover and aging of long-lived postmitotic cells: the mitochondrial-lysosomal axis theory of aging." Antioxid Redox Signal 12(4): 503-535.

Toledano, M. B. and W. J. Leonard (1991). "Modulation of transcription factor NF-kappa B binding activity by oxidation-reduction in vitro." Proc Natl Acad Sci U S A 88(10): 4328-4332.

Toyama, B. H., J. N. Savas, et al. (2013). "Identification of long-lived proteins reveals exceptional stability of essential cellular structures." Cell 154(5): 971-982. 
Tremblay, M. E., R. L. Lowery, et al. (2010). "Microglial interactions with synapses are modulated by visual experience." PLoS Biol 8(11): e1000527.

Tremblay, M. E. and A. K. Majewska (2011). "A role for microglia in synaptic plasticity?" Commun Integr Biol 4(2): 220-222.

Tremblay, M. E., B. Stevens, et al. (2011). "The role of microglia in the healthy brain." J Neurosci 31(45): 16064-16069.

Tremblay, M. E., M. L. Zettel, et al. (2012). "Effects of aging and sensory loss on glial cells in mouse visual and auditory cortices." Glia 60(4): 541-558.

van Rossum, D. and U. K. Hanisch (2004). "Microglia." Metab Brain Dis 19(3-4): 393-411.

Vanlandingham, P. A. and B. P. Ceresa (2009). "Rab7 regulates late endocytic trafficking downstream of multivesicular body biogenesis and cargo sequestration." J Biol Chem 284(18): 12110-12124.

Vitelli, R., M. Santillo, et al. (1997). "Role of the small GTPase Rab7 in the late endocytic pathway." J Biol Chem 272(7): 4391-4397.

Vonderheit, A. and A. Helenius (2005). "Rab7 associates with early endosomes to mediate sorting and transport of Semliki forest virus to late endosomes." PLoS Biol 3(7): e233.

Wake, H., A. J. Moorhouse, et al. (2009). "Resting microglia directly monitor the functional state of synapses in vivo and determine the fate of ischemic terminals." J Neurosci 29(13): 3974-3980.

Walshe, J. M. (1995). "Copper: not too little, not too much, but just right. Based on the triennial Pewterers Lecture delivered at the National Hospital for Neurology, London, on 23 March 1995." J R Coll Physicians Lond 29(4): 280-288.

Wang, S. and K. M. Young (2014). "White matter plasticity in adulthood." Neuroscience 276: 148-160.

Wasserman, J. K., H. Yang, et al. (2008). "Glial responses, neuron death and lesion resolution after intracerebral hemorrhage in young vs. aged rats." Eur J Neurosci 28(7): 1316-1328.

Watkins, T. A., B. Emery, et al. (2008). "Distinct stages of myelination regulated by gamma-secretase and astrocytes in a rapidly myelinating CNS coculture system." Neuron 60(4): 555-569.

Wilmarth, P. A., S. Tanner, et al. (2006). "Age-related changes in human crystallins determined from comparative analysis of post-translational modifications in young and aged lens: does deamidation contribute to crystallin insolubility?" J Proteome Res 5(10): 2554-2566.

Wolf, G. (2003). "Lipofuscin and macular degeneration." Nutr Rev 61(10): 342-346.

Wong, W. T. (2013). "Microglial aging in the healthy CNS: phenotypes, drivers, and rejuvenation." Front Cell Neurosci 7: 1-13.

Yamasaki, R., H. Lu, et al. (2014). "Differential roles of microglia and monocytes in the inflamed central nervous system." J Exp Med 211(8): 1533-1549.

Ye, S. M. and R. W. Johnson (1999). "Increased interleukin-6 expression by microglia from brain of aged mice." J Neuroimmunol 93(1-2): 139-148. 
Yeung, M. S., S. Zdunek, et al. (2014). "Dynamics of oligodendrocyte generation and myelination in the human brain." Cell 159(4): 766-774.

Young, K. M., K. Psachoulia, et al. (2013). "Oligodendrocyte dynamics in the healthy adult CNS: evidence for myelin remodeling." Neuron 77(5): 873-885.

Zerial, M. and H. McBride (2001). "Rab proteins as membrane organizers." Nat Rev Mol Cell Biol 2(2): 107-117.

Zhu, Q., S. R. Whittemore, et al. (2011). "Dorsally-derived oligodendrocytes in the spinal cord contribute to axonal myelination during development and remyelination following focal demyelination." Glia 59(11): 1612-1621.

Ziv, Y., N. Ron, et al. (2006). "Immune cells contribute to the maintenance of neurogenesis and spatial learning abilities in adulthood." Nat Neurosci 9(2): 268-275. 


\section{Curriculum vitae}

\section{Personal data}

Name: Shima Safaiyan

Date of birth: September 23, 1980

Nationality: Iran

Family status: single

\section{Contact information}

Address: Cellular neuroscience department, Max Planck Institute for Experimental Medicine, Hermann Rein Str. 3, 37075, Goettingen, Germany

Email Address: safaiyan@gmail.com

\section{Education}

Aug. 2011-expected Nov. 2015

$\mathrm{PhD}$, Neuroscience, Georg-August University Goettingen

Dissertation: Role of microglia in myelin turnover

Committee: Prof. Dr. Mikael Simons (Advisor), Prof. Dr. Alexander Fluegel, Prof. Dr. Holger Reichardt.

Sept. 2003-Sept. 2006

M.Sc. Microbiology, Tehran University, Iran

Thesis topic: "Screening and Primary Identification of Native Exopolysaccharide-producing Halophilic Bacteria."

Chair: Dr. Mohamad Ali Amoozegar

G.P.A: 18.03 out of 20 
Sept. 1999 to June 2003

B.Sc. Microbiology, Tehran University, Iran

Thesis topic: The Study of Genetics of Mushrooms and Evaluation of Their Biotechnological Applications.

Chair: Dr. Behboodi (Cytology lab, Department of Cellular Biology, Tehran University) G.P.A: 16.29 out of 20

\section{Employment history}

October 2007-February 2011

Research Associate in Molecular Immunology and Vaccine Research Laboratory, Pasteur Institute of Iran

\section{5- Professional Research Experiences}

2008-2011

Molecular Immunology and Vaccine Research Laboratoy, Pasteur Institute of Iran,

Project: The contribution of human neutrophils in development of protective immune responses during in vitro leishmnia major infection" in collaboration with Karolinska institute, Sweden.

2008-2010

Molecular Immunology and Vaccine Research Laboratory, Pasteur Institute of Iran:

Project: The role of, a certain CpG ODN, in modulation of immune responses of human (normal and patients) PBMCs as well as macrophages infected with Leishmania major in vitro. 
Molecular Immunology and Vaccine Research Laboratory, Pasteur Institute of Iran

Project: Assessment of NOD2 Gene polymorphism to find SNPs which have a crucial role in manifestation of different clinical outcomes in humans with Cutaneous Leishmaniasis in Mashhad.

2006, Exteremophiles Lab., Department of Microbiology, Tehran University

Proposal: Using the Exopolymers Produced by Halophilic Bacteria to Absorption of $\mathrm{Pb}^{2+}$ and $\mathrm{Hg}^{2+}$ in polluted environments

Supervisor: Dr. Amoozegar, approved and supported by "National Projects Organization”.

2005, Exteremophiles Lab.,Department of Microbiology, Tehran University.

Proposal: Introducing a new medium for culture of exopolysaccharide-producing halophilic bacteria

2001, Tehran University, Study of cloning procedure in animal cells,

Supervisor, Dr.Anis Jafari (Pasteur Institute of Iran, Molecular Biology Dept.)

\section{6- Publications}

1) Improvement of different vaccine delivery systems for cancer therapy. Bolhassani A, Safaiyan S, Rafati S. Mol Cancer. 2011 Jan 7;10(1):3.

2) In silico Prediction and in vitro Evaluation of Leishmania major Specific Peptides Eliciting HLA class I Restricted CD8+ T cell Response.

Negar Seyed, Farnaz Zahedifard, Shima Safaiyan, Elham Gholami, Fatemeh Doustdari, Kayhan Azadmanesh, Maryam Mirzaei, Nasir Saeedi Eslami, Akbar Khadem Sadegh, Ali Eslami far, Iraj Sharifi and Sima Rafati. PLOS Neglected tropical Diseases, 2011, Sep; 5(9). 
3) Contribution of human neutrophils in the development of protective immune response during in vitro Leishmania major infection. Shima Safaiyan, Azam Bolhassani, Susanne Nylen, Hannah Akuffo, and Sima Rafati. Parasite Immunology 2011, 33: 609-620

4) Prox 1 is Required for Oligodendrocyte Cell Identity in Adult Neural Stem Cells of the Subventricular Zone, Eva C. Bunk, Gökhan Ertaylan, Felipe Ortega, Maria A. Pavlou, Athanasios Stergiopoulos, Shima Safaiyan, Sandra Völs, Marianne van Cann, Panagiotis K. Politis4, Mikael Simons, Benedikt Berninger, Antonio del Sol, Jens C. Schwamborn. (Submitted)

\section{7- Training Course}

Real-Time PCR training course at "International workshop on Leishmania". March $1^{\text {st }}-6^{\text {th }}$, 2008, Pasteur Institute of Iran, Tehran.

\section{Scholarships and grants:}

Grant for Young Researchers in Exteremophiles 2006 Congress: 450€ (2006, France)

\section{Books}

2001: Participation in editing of a Biochemistry book for master-science exam under supervision of Mir publication, Iran

\section{Conference Presentations}

\section{- Poster Presentation}

1- "Evaluation of Viscosity of Exopolysaccharides Produced by Native Moderately Halophilic Bacteria", $8^{\text {th }}$ National Congress of Microbiology. (May 23-25, 2006, Esfahan, Iran)

2- "Screening and Primary Identification of Native Moderately Halophilic EPS-Producing Bacteria", $11^{\text {th }}$ International Symposium on Microbial Ecology, August 20-25, 2006, Vienna Austria

3- "The effect of Physicochemical Factors on Production of Exopolysaccharide by a Halophilic Bacterium Related to Vibrio Genus“, Exteremophiles 2006 Conference, September 17-21 2006, Brest, Brittany, France 
4- "Comparing the effect of different classes of CpG-ODN on human neutrophil survival and activation", $14^{\text {th }}$ International Congress of Immunology, August 22-27. 2010. Kobe, Japan.

5- "connecting myelin membrane turnover with autoimmunity", $2^{\text {nd }}$ International SFB TRR 43 Symposium, May 15 - 17, 2014, Berlin/Potsdam, Germany

\section{- Oral presentation}

1- "Using Taguchi Method for Optimizing the Production of Exopolysaccharide by a Halophilic Bacterium Related to Vibrio Genus.", $14^{\text {th }}$ National and 2th International Conference of Biology, Tehran, Iran, 29-31 August, 2006.

2- "Role of microglia in myelin turnover". Myelintreffen 24-25. April, 2014 Max Planck Institute for Experimental Medicine, Göttingen, Germany 